\title{
The Mori fan of the Dolgachev-Nikulin-Voisin family in genus 2
}

\author{
Klaus Hulek and Carsten Liese
}

\begin{abstract}
In this paper we study the Mori fan of the Dolgachev-Nikulin-Voisin family in degree 2 as well as the associated secondary fan. The main result is an enumeration of all maximal dimensional cones of the two fans.
\end{abstract}

Keywords. K3 surfaces; moduli; minimal model program; mirror symmetry

2020 Mathematics Subject Classification. primary: 14D20, 14J28; secondary: 14E30, 14J33

Received by the Editors on December 13, 2019, and in final form on September 27, 2021.

Accepted on October 25, 2021.

Klaus Hulek

Institut für Algebraische Geometrie, Leibniz Universität Hannover, Welfengarten 1, 30167 Hannover, Germany

e-mail: hulek@math.uni-hannover.de

Carsten Liese

Institut für Algebraische Geometrie, Leibniz Universität Hannover, Welfengarten 1, 30167 Hannover, Germany

e-mail: liese@math.uni-hannover.de

We are grateful to DFG for financial support under grant $\mathrm{Hu} 337 / 7-1$.

(C) by the author(s)

This work is licensed under http://creativecommons.org/licenses/by-sa/4.0/ 


\section{Contents}

1. Introduction. . . . . . . . . . . . . . . . . . . . . . . . . . . . . . . . 2

2. The Dolgachev-Nikulin-Voisin mirror of degree $2 d$ : definition and construction . . . . $\quad 5$

3. Relative Notions and the Mori fan . . . . . . . . . . . . . . . . . . . . . . . 19

4. (-1)-Curves and Curve Structures . . . . . . . . . . . . . . . . . . . . . . . 22

5. Projective Models . . . . . . . . . . . . . . . . . . . . . . . . . . . . . 32

6. Flops, Birational Automorphisms and Orbits. . . . . . . . . . . . . . . . . . . 35

7. Counting models . . . . . . . . . . . . . . . . . . . . . . . . . . . . . . 50

8. The Secondary fan . . . . . . . . . . . . . . . . . . . . . . . . . . . . . 59

References. . . . . . . . . . . . . . . . . . . . . . 65

\section{Introduction}

To construct modular compactifications of the moduli space $\mathcal{F}_{2 d}$ of polarized $K 3$ surfaces of degree $2 d$ is a notoriously difficult problem. This has been studied from various aspects, such as Hodge theory, locally symmetric domains, GIT and log-geometry. For small degree $d=1,2,3$ these $K 3$ surfaces can be studied via concrete geometric models, namely $2: 1$ covers of the projective plane $\mathbb{P}^{2}$ branched along a sextic curve, degree 4 surfaces in $\mathbb{P}^{3}$ and complete $(2,3)$ intersections in $\mathbb{P}^{4}$ respectively. Various authors have used this approach to construct compactifications of $\mathcal{F}_{2 d}$ in these degrees and to relate the various models to each other. Here we would like to mention in particular the works of Shah [Sha80], Friedman [Fri83], Looijenga [Loo86], Laza [Laz08, Laz16], Laza-O’Grady [LO18, LO16, LO21], Thompson [Tho14] and Alexeev-Engel-Thompson [AET19].

Some years ago Gross, Hacking, Keel and Siebert [GHKS] introduced a new approach. This is based on two main concepts: mirror symmetry and the minimal model program (MMP). They start by considering the mirror family of $\mathcal{F}_{2 d}$. This is a 1-dimensional family of lattice polarized $K 3$ surfaces which in the literature is called the Dolgachev-Nikulin-Voisin family of degree $2 d$. The base of this family is a modular curve which, if $d$ is squarefree, has exactly one cusp. The lattice polarization is given by the lattice $\check{M}_{2 d}=U \oplus 2 E_{8}(-1) \oplus\langle-2 d\rangle$ where $U$ is the hyperbolic plane and $E_{8}(-1)$ is the negative definite $E_{8}$ lattice. The first step in their programme is to extend the Dolgachev-Nikulin-Voisin family over the cusp and to consider the various models by which this can be done. This allows them to define the Mori fan of the Dolgachev-Nikulin-Voisin family, which is a fan in $\mathrm{N}^{1}(\mathcal{Y} / S)_{\mathbb{R}}$, where $\mathcal{Y} \rightarrow S$ is a model of the DolgachevNikulin-Voisin family, a scheme of dimension $19+d$, with base $S=\operatorname{Spec} \mathbb{C}[[t]]$. The second step is to use a piecewise linear section of the restriction map $r: \operatorname{Pic}(\mathcal{Y}) \rightarrow \operatorname{Pic}\left(\mathcal{Y}_{\eta}\right)$, where $\mathcal{Y}_{\eta}$ the Dolgachev-Nikulin-Voisin family over a punctured neighbourhood of the cusp, to obtain a fan in the hyperbolic space $\left(\check{M}_{2 d}\right)_{\mathbb{R}}$. An important aspect of their work is the construction of a universal family (at least over a neighbourhood of the 0 -dimensional cusps). A detailed description of the GHKS programme for $K 3$ surfaces is contained in [GHKS]. Zhu [Zhu18] has carried this out in the case of polarized abelian varieties and has shown that this approach can be used to recover the second Voronoi compactifiation of $\mathcal{A}_{g}$, which is known to be a modular compactification by the work of Alexeev [Ale02] and Olsson [Ols12]. 
Our aim is to start a concrete investigation of the GHKS approach for small degree. To be precise, we want to understand the first step in the GHKS programme in degree 2. As it turns out this is a nontrivial problem in its own right. The main result of our paper is a concrete description of the Mori fan of the Dolgachev-Nikulin-Voisin family in degree 2. Concretely, we enumerate the maximal dimensional cones and prove the following:

Theorem 1.1. Let $\mathcal{Y} \rightarrow S$ be a model of the Dolgachev-Nikulin-Voisin family of degree 2. Then $\mathrm{MF}(\mathcal{Y} / S)$ has 3460 maximal cones. Of these 753 are associated to a model of class $\mathscr{T}$ and 2707 are associated to a model of class $\mathscr{P}$. The number of orbits of maximal cones of $\mathrm{MF}(\mathcal{Y} / S)$ under the natural action of the birational group $\operatorname{Bir}(\mathcal{Y} / S)$ is 588. The orbits decompose into 457 orbits of models of class $\mathscr{P}$ and 131 orbits of models of class $\mathscr{T}$.

This is a consequence of Theorems 7.7, 7.13, and 7.15. Here class $\mathscr{P}$ and class $\mathscr{T}$ refer to the combinatorial structure of the central fibre, which in turn correspond to the two possible triangulations of the sphere $S^{2}$ into two triangles.

As we explained earlier, the GHKS programme aims at constructing (semi-)toroidal compactifications of moduli spaces of polarized $K 3$ surfaces. This means constructing (semi-)fans covering the rational closure of the real positive cone $C^{+}\left(\check{M}_{2 d}\right)$. Such fans consist of infinitely many cones, decomposing into finitely many orbits under the action of the orthogonal group $\mathrm{O}^{+}\left(\check{M}_{2 d}\right)$. This construction (see [GHS16, Sections 6-8] and [HLL20, Section 5] for more details) is based on the Mori fan $\operatorname{MF}(\mathcal{Y} / S)$. In our paper we work with a fixed model $\mathcal{Y} / S$ for degree $2 d=2$ (but our results are independent of the choice of such a model). This eventually leads to finitely many cones contained in a fixed Weyl chamber in the positive cone $C^{+}\left(\check{M}_{2 d}\right)$. The action of the Weyl group of $\mathrm{O}\left(\check{M}_{2}\right)$ then gives rise to infinitely many cones. We would also like to point out that the lattice $\check{M}_{2 d}$ is a reflexive lattice if and only if $2 d=2$. Equivalenty, $\operatorname{Bir}(\mathcal{Y} / S) \cong \operatorname{Aut}\left(\mathcal{Y}_{\eta}\right)$ is finite if and only if $2 d=2$ (in which case it is isomorphic to $S_{3}$ ). In this case the Weyl chamber is rational polyhedral.

We also investigate the so called secondary fan which was used by Hacking, Keel and Yue [HKY20] and is a generalisation of the secondary fan for toric varieties due to Gelfand-Kapranov-Zelevinskij [GKZ94]. This is a coarsening of the Mori fan. Its relevance is that in the del Pezzo case the toric variety defined by the secondary fan admits a finite morphism to the moduli space of stable pairs. Note that for toric varieties, the secondary fan and the Mori fan coincide.

As there is no published proof available that the secondary fan is indeed a fan in the $K 3$ setting, we will include a proof of this fact using the techniques of our paper and we will also compute its maximal cones:

Theorem 1.2. Let $\mathcal{Y} \rightarrow S$ be a model of the Dolgachev-Nikulin-Voisin family of degree 2. The secondary fan contains precisely 4 maximal cones. There are 2 orbits of maximal cones under the natural action of $\operatorname{Bir}(\mathcal{Y} / S)$.

This is Theorem 8.10 and Remark 8.12.

We shall now briefly describe the structure of this paper. We start in Section 2 by recalling the theory of mirror symmetry for lattice polarized K3 surfaces, which is due to Dolgachev, Nikulin and Pinkham.

We then recapitulate the basics of the degeneration theory of $K 3$ surfaces as developed by Carlson, Friedman, Kulikov, Persson, Pinkham, Scattone and others. This allows us to define the notion of the Dolgachev-Nikulin-Voisin family of degree $2 d>0$ and their models in Definitions 2.20 and 2.22, following [GHKS]. We further recall the relation between triangulations of the sphere $S^{2}$ and $d$-semistable models in $(-1)$-form of the Dolgachev-Nikulin-Voisin family of degree $2 d$ with maximal Picard rank for which we describe the geometry of the special fibres in detail (Construction 2.25). We also prove that these surfaces and their maximal smoothings are projective (Propositions 2.27 and 2.28). Finally we discuss the (-1)-models in degree 2 in detail. In Section 3 we start by recalling some basic facts of the minimal model program and introduce the main object of this paper, the Mori fan of the Dolgachev-Nikulin-Voisin family (Definition 3.2). We describe its main properties in (Proposition 3.4), due to the work of Gross, Hacking, Keel and Siebert. 
Next we explain the relationship between interior facets and flops (Proposition 3.9) and finally discuss the action of the group of birational automorphisms on the Mori fan (Proposition 3.10).

In Section 4 we mostly specialise to degree 2. Corresponding to the two triangulations of the sphere $S^{2}$ with two triangles we have two possible $d$-semistable $K 3$ surfaces in $(-1)$-form, which we denote $Y_{\mathscr{P}}$ and $Y_{\mathscr{T}}$ respectively. We shall consider all models whose central fibres can be transformed by a series of type I flops into $Y_{\mathscr{P}}$ or $Y_{\mathscr{T}}$, and call these models of type $\mathscr{P}$ and type $\mathscr{T}$ respectively. As we shall see later, see Corollary 6.33, these are all models in degree 2. The main object of this section is a detailed analysis of the configuration of certain curves forming an anticanonical divisor on components of the central fibre of a given model of the Dolgachev-Nikulin-Voisin family. This leads to the notion of curve structure, see Definition 4.10 and will also provide us with a natural $\mathbb{Q}$-basis of the Picard group of the normalisations of the central fibre (Proposition 4.17). The main application will be existence theorems of ample line bundles of prescribed degree on the components of the anticanonical divisor (Propositions 4.30, 4.31 and 4.31 ). This discussion will become vital in Section 5 where we prove projectivity criteria for models of type $\mathscr{T}$ (Proposition 5.1) and type $\mathscr{P}$ (Propositions 5.2, 5.3, 5.4 and 5.5 ).

Section 6 is in many ways the technical heart of the paper. Here we analyse flops between models of the Dolgachev-Nikulin-Voisin family in some detail and study the action of the birational automorphism group on the Mori fan. For this we introduce various concepts describing (augmented) curve structures (see Definitions 6.17, 6.18 and Construction 6.23). This allows us to establish two crucial facts about models of the Dolgachev-Nikulin-Voisin family in degree 2. The first we have already mentioned above, namely that any such model can be related by type I flops to a model of type $\mathscr{P}$ (or equivalently $\mathscr{T}$ ) (see Corollary 6.33). The second is that any two models of the Dolgachev-Nikulin-Voisin family can be transformed into each other using only type I and type II flops (Corollary 6.34). We note that these results are specific to degree 2. Finally, this enables us to study the action of the birational automorphism group of a model of the Dolgachev-Nikulin-Voisin family on the Mori fan. In Proposition 6.43 we determine the possible orbit lengths of maximal cones of this fan under the birational automorphism group, which can be 1,3 or 6 .

In Section 7 we finally enumerate all models of the Dolgachev-Nikulin-Voisin family in degree 2 and determine the maximal cones in the Mori fan (Theorem 7.15). This section is rather combinatorial in nature. To obtain our result we use the tools which we have developed before, in particular we use curve structures: these allow us to characterise the isomorphism classes of the central fibres of projective models of the Dolgachev-Nikulin-Voisin family. This allows us an explicit enumeration of the models. The second ingredient is the action of the birational automorphism group and its action on the set of maximal cones in the Mori fan which we analysed in the previous section. The main result then follows from a careful enumeration of all models, which we do for types $\mathscr{P}$ (Theorem 7.8) and $\mathscr{T}$ (Theorem 7.7) separately. In Section 8 we finally describe the secondary Mori fan. In this section we give a fairly elementary proof that the secondary fan is indeed a fan and compute its maximal cones (Theorem 8.10).

Throughout the paper we will work over the complex numbers $\mathbb{C}$.

\section{Acknowledgments}

We have benefited enormously from discussions with Christian Lehn. We thank Mark Gross, Paul Hacking, Sean Keel, Bernd Siebert and Tony Yu Yue for sharing drafts of [GHKS] and [HKY20] and for answering our questions. We also thank two referees for their careful reading and helpful suggestions. These not only improved the presentation considerably, but also helped us correct an error in the first version of the paper. 


\section{The Dolgachev-Nikulin-Voisin mirror of degree $2 d$ : definition and construction}

\subsection{The Dolgachev-Nikulin-Voisin mirror of degree $2 d$}

We first recall the mirror construction due to Dolgachev [Dol96] and Pinkham [Pin77] for polarized K3 surfaces, using Nikulin's theory of lattice polarized K3 surfaces [Nik79]. This was motivated by the papers by Lian and Yau [LY96a, LY96b]. A further contribution using $K 3$ surfaces is due to Voisin [Voi93]. For the basic facts about $K 3$ surfaces and their moduli which we will need, we refer the reader to e.g. [Huyl6], [BHPV04, Section VIII] and [GHS15]. The second cohomology group of a $K 3$ surface, together with the cup product (intersection pairing), define a lattice which is isomorphic to the K3-lattice

$$
L_{K 3}=2 E_{8}(-1) \oplus 3 U
$$

where $U$ is the hyperbolic plane and $E_{8}(-1)$ is the even negative definite unimodular lattice of rank 8 . A polarization on $X$ is an ample line bundle $\mathcal{L}$ and, since $K 3$ surfaces are regular, we can identify a polarization with its first Chern class $h=c_{1}(\mathcal{L}) \in H^{2}(X, \mathbb{Z})$. We assume $h$ to be primitive and of degree $h^{2}=2 d>0$. We note that the group of isometries of the $K 3$ lattice operates transitively on the set of primitive vectors of given positive degree. Instead of working with the degree of a polarization we will often also use its genus, by which we mean the genus of a general element in the linear system defined by $\mathcal{L}$. The genus $g$ and the degree $2 d$ are related by the adjunction formula $2 g-2=2 d$. Note that degree 2 coincides with genus 2 .

The moduli theory of $K 3$ surfaces builds on the Torelli theorem. To describe this, we first notice that the orthogonal complement of $h$ in $L_{K 3}$ defines a lattice

$$
L_{2 d} \cong 2 E_{8}(-1) \oplus 2 U \oplus\langle-2 d\rangle .
$$

We obtain the period domain $\Omega_{2 d}$ by

$$
\Omega_{2 d}=\left\{x \in \mathbb{P}\left(L_{2 d} \otimes \mathbb{C}\right) \mid x^{2}=0,\langle x \bar{x}\rangle>0\right\} .
$$

This is a 19-dimensional manifold which has two connected components of which we fix one, say $D_{2 d}$. A quasi-polarized $K 3$ surface is a pair $(X, \mathcal{L})$ where $\mathcal{L}$ is big and nef. Recall that a multiple of $\mathcal{L}$ will embed $X$ as a $K 3$ surface with ADE-singularities. It is a classical application of the Torelli theorem that the moduli space of degree $2 d$ polarized $K 3$ surfaces with ADE-singularities is isomorphic to the quotient

$$
\mathcal{F}_{2 d}=\Gamma_{2 d} \backslash \Omega_{2 d}=\Gamma_{2 d}^{+} \backslash D_{2 d}
$$

where

$$
\Gamma_{2 d}=\left\{g \in \mathrm{O}\left(L_{K 3}\right) \mid g(h)=h\right\}
$$

is the group of all isometries of $L_{K 3}$ which fix the polarization $h$ and $\Gamma_{2 d}^{+}$is the subgroup of elements of real spinor norm 1 (which is equivalent to the property that these elements fix the components of $\Omega_{2 d}$.) To obtain the moduli space of all polarized $K 3$ surfaces one has to remove finitely many hyperplanes from $\mathcal{F}_{2 d}$, see e.g. [BHPV04, p. 355].

To simplify the following discussion we will now assume that $d$ is square free. Then we have a well defined mirror moduli space which was described in [Dol96, §6]. This parameterizes lattice polarized $K 3$ surfaces of Picard rank 19 whose Picard lattice is isomorphic to

$$
\check{M}_{2 d}=U \oplus 2 E_{8}(-1) \oplus\langle-2 d\rangle
$$

and we note that

$$
L_{2 d}=h_{L_{K 3}}^{\perp}=\check{M}_{2 d} \oplus U
$$


By Nikulin's theory the lattice $\check{M}_{2 d}$ has a unique primitive embedding into the $K 3$ lattice $L_{K 3}$ (up to isometries), and here we fix once and for all the obvious embedding, which maps a generator of the summand $\langle-2 d\rangle$ to $e-d f$ in a summand $U$, where $e, f$ are a basis of $U$ with $e^{2}=f^{2}=0$ and $e \cdot f=1$. Similar to above, this leads to the moduli space

$$
\check{\mathcal{F}}_{2 d}=\check{\Gamma}_{2 d} \backslash \Omega_{\left(\check{M}_{2 d}\right)_{L_{K 3}}^{\perp}}
$$

where

$$
\check{\Gamma}_{2 d}=\left\{g \in \mathrm{O}\left(L_{K 3}\right)|g|_{\check{M}_{2 d}}=i d\right\}
$$

is now the group of all isometries of $L_{K 3}$ which restrict to the identity on $\check{M}_{2 d}$. In our case

$$
\left(\check{M}_{2 d}\right)_{L_{K 3}}^{\perp}=U \oplus\langle 2 d\rangle
$$

and this is dual to relation (2.1). The period domain $\Omega_{\left(\check{M}_{2 d}\right)_{L_{K 3}}^{\perp}}$ is 1-dimensional, more precisely it is two copies of the upper half plane $\mathbb{H}_{1}$ (which are interchanged by the group $\check{\Gamma}_{2 d}$ ). Hence $\check{\mathcal{F}}_{2 d}$ is a connected (non-compact) modular curve. Mirror symmetry interchanges the roles of complex moduli and Kähler moduli. This corresponds to the fact that the mirror moduli space $\check{\mathcal{F}}_{2 d}$ is one dimensional and that the very general $K 3$ surface in $\check{\mathcal{F}}_{2 d}$ has Picard group $\check{M}_{2 d}$.

Since we assumed that $d$ is square-free it follows from Scattone's calculations in [Sca87, §4], that there is a unique 0-dimensional boundary component in the Baily-Borel compactification of $\mathcal{F}_{2 d}$. By [Dol96, Proposition 7.3] the same is true for $\check{\mathcal{F}}_{2 d}$. The mirror family which we are interested in is the universal family over $\check{\mathcal{F}}_{2 d}$ near the cusp. This requires an explanation. The moduli spaces $\mathcal{F}_{2 d}$ and $\check{\mathcal{F}}_{2 d}$ do not carry universal families in the category of schemes (due to the existence of non-trivial automorphisms). Nevertheless, this concept can be made precise in the neighbourhood of the cusp and we will do this below where we define the Dolgachev-Nikulin-Voisin mirror family in a rigorous way, see Definition 2.20. In what follows, we will typically work in the following situation. Let $(R, m)$ be a local complete DVR with residue field $k=R / m$. We will always assume here that $k=\mathbb{C}$. Let $K=\mathrm{Q}(R)$ be the field of fractions of $R$. We set $S=\operatorname{Spec}(R)$. Typically we will work with the completion $R=\hat{\mathcal{O}}_{C, p}$ of the local ring of an affine curve. We denote by $0=\operatorname{Spec}(k)$ the closed point of $S$ and by $\eta=\operatorname{Spec}(K)$ the generic point of $S$. It will essentially be enough to consider the case $R=\mathbb{C}[[t]]$ of formal power series whose field of fractions is the field $K=\mathbb{C}((t))$ of Laurent series. Indeed, if $(C, p)$ is a curve germ, then we can choose a local parameter $\pi$, and this defines an isomorphism of $k$-algebras $R \rightarrow \hat{\mathcal{O}}_{C, p}$. If $\mathcal{Y} \rightarrow S$ is a scheme over $S$, then we denote the generic fibre by $\mathcal{Y}_{\eta}$ and the special (central) fibre by $\mathcal{Y}_{c}$. Alternatively, we can also work in the analytic category and consider families $\mathcal{Y} \rightarrow \mathbb{D}$ over the disc and their restriction to the origin $0 \in \mathbb{D}$ and the punctured disc $\mathbb{D}^{*}$ respectively. We will sometimes use the analytic category in proofs.

In this paper we will use the term normal crossing to denote a scheme which is locally (not necessarily globally) normal crossing with reduced components. We say that a normal crossing scheme $Y$ is smoothable if there exists a regular scheme $\mathcal{Y}$, a proper flat map $\mathcal{Y} \rightarrow S$ and an isomorphism $\mathcal{Y}_{c} \cong Y$. In this case the restriction of the normal bundle of $\mathcal{Y}_{c}$ in $\mathcal{Y}$ to the singular locus $D$ of $\mathcal{Y}_{c}$ is trivial:

$$
N_{D}:=\left.N_{\mathcal{Y}_{c} / \mathcal{Y}}\right|_{D}=\left.\mathcal{O}_{\mathcal{Y}}\left(\mathcal{Y}_{c}\right)\right|_{D} \cong \mathcal{O}_{D} \text {. }
$$

The line bundle $N_{D}$ is called the infinitesimal normal bundle and can also be defined purely in terms of the singular scheme $Y$ by using the normal bundle of the components of $D$ in the respective components of $Y$, see [Fri83, §1]. We say that $Y$ is $d$-semistable if $N_{D}$ is trivial which is a non-trivial condition if we just consider an abstract surface $Y$. The triviality of $N_{D}$ is a necessary condition for smoothability of $Y$. It was shown by Friedman that it is also sufficient [Fri83, Theorem 5.10].

We will now recall the basic facts about degenerations of $K 3$ surfaces in so far as they are relevant for us. This goes back to the fundamental results in this area as it was developed in the seminal work of Kulikov [Kul77] and Persson and Pinkham [PP81] in the late 1970's and early 1980's. We will denote by $Y$ a proper normal crossing surface and by $Y_{i}$ the components of $Y$. If $Y$ is a simple normal crossing (snc) surface, then 
the components $Y_{i}$ are smooth. We will, however, also allow self-intersections of the components and we will denote the normalisation of a component $Y_{i}$ by $Y_{i}^{v}$. As before, we will denote the singular locus of $Y$ by $D$. We set $D_{i j}=Y_{i} \cap Y_{j}$ and consider this as a curve on $Y_{i}$. We also allow $i=j$ and in this case $D_{i i}$ denotes the self-intersection of the component $Y_{i}$. In our case the curves $D_{i j}$ will always be irreducible. Intersection numbers $D_{i j}^{2}$ will always be calculated on the normalisations $Y_{i}^{v}$. Note that this may depend on the ordering of $\{i, j\}$.

There are three types of semi-stable degenerations of $K 3$ surfaces, classically called type I, II and III. The type of a degeneration is a measure of how far the Hodge structure degenerates. Type I are smooth K3 surfaces. The building blocks of type II degenerations are rational and elliptically ruled surfaces and the curves $D_{i j}$ consist of elliptic curves (which still carry some Hodge structure). The components of type III degenerations are rational surfaces, intersecting in curves whose components are also rational. Another characterization of the type can be given in terms of the nilpotency of the monodromy, see e.g. [Sca87, §1.2].

Our interest will be in type III degenerations. The following definitions are fundamental:

Definition 2.1 ( $c f$. [Fri83, Definition 5.5]). A d-semistable K3 surface of type III is a normal crossing surface $Y$ such that

(i) $Y$ is $d$-semistable

(ii) $\omega_{Y}=\mathcal{O}_{Y}$

(iii) $Y=\cup Y_{i}$ where each $Y_{i}$ is rational and the preimage of the double curves $\sum_{j} D_{i j}$ form anticanonical cycles of rational curves on the normalisation $Y_{i}^{v}$

(iv) The dual intersection complex of $Y$ is a triangulation of the 2-sphere $S^{2}$.

In the projective situation we make the following

Definition 2.2. A type III degeneration of $K 3$ surfaces is a flat, projective scheme $\mathcal{Y} \rightarrow S$ over the spectrum $S$ of a complete DVR, where $\mathcal{Y}$ is a regular 3 -fold whose generic fibre $\mathcal{Y}_{\eta}$ is a $K 3$ surface and whose central fibre $\mathcal{Y}_{c}$ is a type III $d$-semistable $K 3$ surface. We will also refer to such a family $\mathcal{Y} \rightarrow S$ as a Kulikov model.

Remark 2.3. Note that the total space $\mathcal{Y}$ is Calabi-Yau, i.e. $\omega_{\mathcal{Y}}=\mathcal{O}_{\mathcal{Y}}$.

Remark 2.4. By definition the central fibre of a Kulikov model is always projective. We note, however, that a priori Definition 2.1 does not require $Y$ to be projective. We further remark that classically Kulikov models were defined in the analytic category. Contrary to this, our default convention will be that we work in the algebraic category. We will, however, occasionally also use the analytic category, in which case we say so explicitly.

Remark 2.5. One can also consider (not necessarly projective) analytic smoothings. In the analytic category, a type III degeneration of $K 3$ surfaces is a morphism $\mathcal{X} \rightarrow \mathbb{D}$, with $\mathbb{D}$ a small disc, such that the general fibres are smooth $K 3$ surfaces and the central fibre is a type III $d$-semistable $K 3$ surface as above. In particular, such an $\mathcal{X}$ is smooth. Every $d$-semistable surface admits an analytic smoothing by [Fri83, Theorem 5.10].

Next, we discuss certain modifications of $d$-semistable $K 3$ surfaces, the elementary modifications. Recall that an F-flopping contraction of a threefold $\mathcal{Y} \rightarrow S$ with trivial canonicial class, where $F$ is a $\mathbb{Q}$-Cartier divisor, is a proper birational contraction $f: \mathcal{Y} \rightarrow \mathcal{Z}$ to a normal scheme (or complex analytic space) $\mathcal{Z} \rightarrow S$ such that the exceptional locus is of codimension at least 2 and the divisor $-F$ is $f$-ample. An $F-f l o p$ of $\mathcal{Y} \rightarrow S$ is a scheme $\mathcal{Y}^{+} \rightarrow S$ together with a proper birational morphism $f^{+}: \mathcal{Y}^{+} \rightarrow \mathcal{Z}$ such that the birational transform $F^{+}$of $F$ is $\mathbb{Q}$-Cartier on $\mathcal{Y}^{+}$and $F^{+}$is $f^{+}$-ample and the exceptional locus of $f^{+}$has codimension at least 2. The induced birational map $\phi: \mathcal{Y} \rightarrow \mathcal{Y}^{+}$is, by abuse of language, also called a flop 


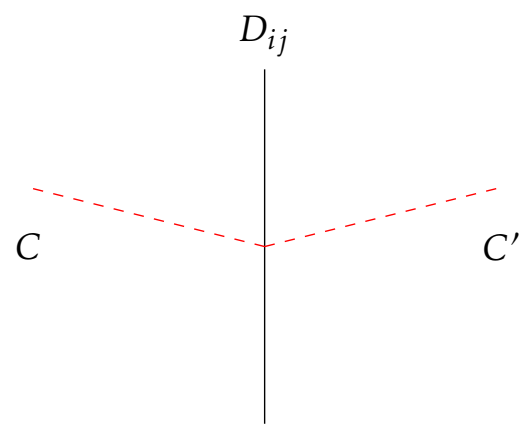

Figure 1. An elementary modification of type I

of $\mathcal{Y}$. The situation can be summarized by the following diagram

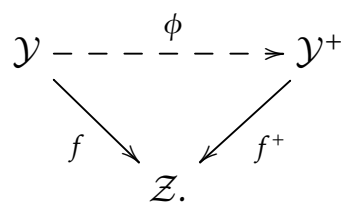

If $\mathcal{Y} \rightarrow \mathcal{Z}$ is the contraction of an extremal ray, then the $F$-flop is independent of the choice of $F$, see [KM98, §6.1]. In this paper, we will always assume that a flop is given by a contraction of an extremal ray.

One can also consider flops in the analytic category. Here we will recall certain types of analytic flops, the elementary modifications. There are three types of these, classically known as type 0, I and II [FM83]. Here we will only be concerned with elementary modifications of type I and II. For the convenience of the reader we will recall these here. Let $Y$ be a $d$-semistable $K 3$ surface as above. Let $\mathcal{X} \rightarrow \mathbb{D}$ be an analytic smoothing over a small disc. Let $C \subset Y$ be a smooth rational curve which intersects the double locus $D$ in exactly one point, more precisely, transversally in a point of some $D_{i j}$ which is a smooth point of $D$. The curve $C$ lies on a unique component $Y_{i}$ and we assume that $C^{2}=-1$ on the normalisation $Y_{i}^{v}$. Then one can blow up $\mathcal{X}$ in $C$ and the exceptional divisor will be isomorpic to $\mathbb{P}^{1} \times \mathbb{P}^{1}$ with normal bundle $\mathcal{O}_{\mathbb{P}^{1} \times \mathbb{P}^{1}}(-1,-1)$. The blow-down map contracts one ruling of the exceptional divisor to $C$. Contracting the other ruling gives another model $\mathcal{X}^{\prime} \rightarrow \mathbb{D}$. The exceptional divisor contracts to a curve $C^{\prime}$ on $Y_{j}$ and we have flopped the curve $C$ to $Y_{j}$. The curve $C^{\prime}$ is again a $(-1)$ curve on $Y_{j}^{v}$. Here we allow $Y_{j}$ and $Y_{i}$ to coincide. This defines an elementary modification of type I, see Figure 1. This construction induces a modification $\psi: Y \rightarrow Y^{\prime}$ of the central fibre $Y$. Following standard terminology we will also refer to this induced map on the central fibre as an elementary modification of type $I$.

Alternatively, let $C$ be a smooth rational component of the double curve $\sum_{j} D_{i j}$ with $C^{2}=-1$ on both $Y_{i}^{v}$ and $Y_{j}^{v}$, where we again allow the components to coincide. Blow up $\mathcal{X}$ in $C$; the resulting exceptional divisor will again be $\mathbb{P}^{1} \times \mathbb{P}^{1}$. As before, we can contract the other ruling to obtain a degeneration $\mathcal{X}^{\prime} \rightarrow \mathbb{D}$, yielding an elementary modification of type II, see Figure 2. Again, one obtains a modification $Y^{\prime}$ of the surface $Y$ to which we will also refer to as an elementary modification of type II.

By the nature of birational geometry for threefolds, for each Kulikov model, there are in general many birational Kulikov models. One can, however, pick out a special class of such models, namely the Kulikov models in (-1)-form.

Definition 2.6. Let $Y$ be a type III $d$-semistable $K 3$ surface. Then we say that $Y$ is in $(-1)$-form if for each smooth double curve $D_{i j}=Y_{i} \cap Y_{j}$, we have $D_{i j}^{2}=D_{j i}^{2}=-1$ and if $D_{i j}$ is singular (and hence a nodal rational curve), then $D_{i j}^{2}=1$ and $D_{j i}^{2}=-1$.

We will call a Kulikov model $\mathcal{Y} \rightarrow S$ with central fibre $Y$ in (-1)-form a Kulikov model in (-1)-form. Note that by a theorem of Miranda and Morrison [MM83, Main Theorem 1.2] any analytic type III Kulikov model 


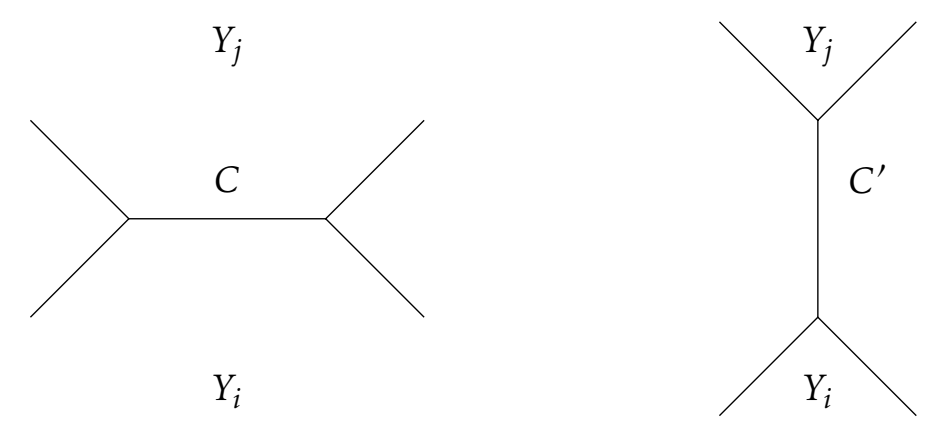

Figure 2. An elementary modification of type II

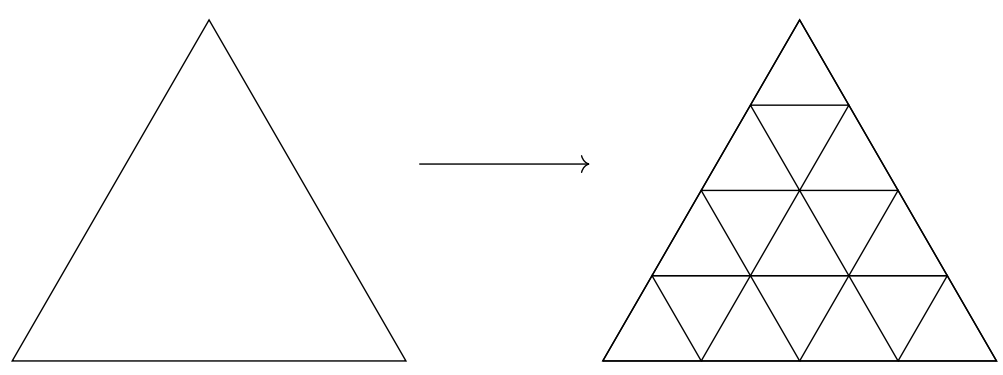

Figure 3. The refinement of a triangle of $\Gamma^{\prime}$.

can, by a series of elementary modifications of type I and II, be brought into (-1)-form, but that (-1)-forms are still not unique. As discussed above, one can also think of this sequence of modifications as a sequence of modifications on the central fibre. Thus one may interpret the theorem of Miranda and Morrison as a result on type III $d$-semistable $K 3$ surfaces.

Theorem 2.7 ( $c f$. [MM83, Main Theorem 1.2]). Let $Y$ be a d-semistable K3 surface of type III. Then there is a sequence $Y \rightarrow Z$ of elementary modifications of type I and II such that $Z$ is a d-semistable K 3 surface in $(-1)$-form.

For future use we also want to recall the index of the monodromy. Let $Y$ denote a $d$-semistable $K 3$ surface in $(-1)$-form, let $\Gamma$ be its dual graph, a triangulation of the sphere $S^{2}$. We say $Y$ has special $n$-bands of hexagons if $\Gamma$ is a refinement of another triangulation $\Gamma^{\prime}$ of $S^{2}$, and is in fact obtained from $\Gamma^{\prime}$ by subdividing each triangle of $\Gamma^{\prime}$ into $n^{2}$ triangles, see Figure 3 for $n=4$.

Now let $Y$ be any $d$-semistable $K 3$ surface of type III. Let $Y^{\prime}$ denote a $d$-semistable $K 3$ surface in $(-1)$-form that is obtained from $Y$ by a sequence of elementary modifications. Let $k$ be the largest integer such that $Y^{\prime}$ has special $k$-bands of hexagons. Note that this number is independent of the choice of $Y^{\prime}$ by [FS86, Theorem 0.5].

Definition 2.8. The integer $k$ is called the index of $Y$. If $k=1, Y$ is called primitive.

Remark 2.9. The index is an invariant of $Y$ that may also be defined in terms of monodromy in the analytic setting. For details see [FS86, p. 4].

We finally have to recall some facts about the Picard group of $d$-semistable $K 3$ surfaces and their smoothings. We first start with a normal crossing surface $Y=\cup Y_{i}$ fulfilling the conditions of Definition 2.1 with possibly the exception that $Y$ is $d$-semistable. Then there is an exact sequence

$$
0 \rightarrow L \rightarrow \bigoplus_{i} H^{2}\left(Y_{i}^{v}, \mathbb{Z}\right) \rightarrow \bigoplus_{C} H^{2}(C, \mathbb{Z})
$$


where $C$ runs through all components of preimages of $D$ under the normalisation $Y^{v} \rightarrow Y$ and $L$ is defined as the kernel of the right hand map given by the differences of the restrictions, see e.g. [KK98, p. 151]. By [Laz08, Section 3.1], $\operatorname{rank} L=18+n$, with $n$ the number of components of $Y$. Obviously, $\operatorname{Pic}(Y) \subset L$. Recall also from [Car80, §4] the Carlson homomorphism

$$
c_{Y}: L \rightarrow \mathbb{C}^{*} .
$$

One way to define it is as follows, see [FS86, §3]: let $W_{0}=W_{1} \subset W_{2}$ be the weight filtration of the natural mixed Hodge structure on $H^{2}(Y)$. We have $L=\left(W_{2} / W_{0}\right)_{\mathbb{Z}}$. Choose a section $s$ of the projection $W_{2} \rightarrow W_{2} / W_{0}$ preserving the Hodge filtration and a retraction $r: W_{2} \rightarrow W_{0}$ of the inclusion $W_{0} \subset W_{2}$. Then $c_{Y}=r \circ s \bmod \left(W_{0}\right)_{\mathbb{Z}}: L \rightarrow\left(W_{0}\right)_{\mathbb{C}} /\left(W_{0}\right)_{\mathbb{Z}}$.

Its significance is that

$$
\operatorname{Pic}(Y)=\operatorname{ker}\left(c_{Y}\right)
$$

We note that for each component $Y_{i}$, there is an element $\xi_{i}=\sum_{j} D_{i j}-D_{j i} \in L$. By results of Friedman and Scattone [FS86, p. 25], $Y$ is smoothable if and only if $\xi_{i} \in \operatorname{ker}\left(c_{Y}\right)$ for all $i$.

We can then consider locally trivial deformations of $Y$, i.e. deformations $X \rightarrow B$, with $B$ an analytic

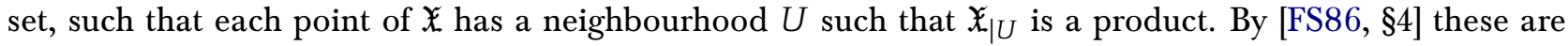
parameterized by the Carlson map $c_{Y}$. The dimension of the family of locally trivial deformations is $18+n$, where $n$ is the number of components of $Y$. The condition $d$-stability imposes $n-1$ conditions, giving a 19-dimensional family. From the relation (2.4) we obtain in particular the following result.

Lemma 2.10. Let $Y=\cup Y_{i}$ be a d-semistable K3 surface of type III with $c_{Y}=1$. Then

$$
\operatorname{Pic}(Y) \cong\left\{\left(\mathcal{L}_{i}\right)_{i} \in \bigoplus_{i} \operatorname{Pic}\left(Y_{i}^{v}\right) \mid \operatorname{deg} \mathcal{L}_{i_{\mid C}}=\operatorname{deg} \mathcal{L}_{j_{\mid C}}, C \subset D^{v}\right\}
$$

where $D^{v}$ denotes the preimage of $D$ under the normalisation map $v: Y^{v} \rightarrow Y$ and $C$ runs through all components of $D$.

The following lemma is an application of [FS86, Lemma 5.5].

Lemma 2.11. Let $Y$ be a d-semistable $K 3$ surface with $n$ components. There is a unique locally trivial d-semistable deformation $Y_{c}$ of $Y$ such that the inclusion $\operatorname{Pic}\left(Y_{c}\right) \rightarrow L$ defines an isomorphism $\operatorname{Pic}\left(Y_{c}\right) \cong L$. This is equivalent to the property that the Carlson homomorphism $c_{Y_{c}}$ is trivial, i.e. $c_{Y_{c}}=1$.

Proof. A detailed proof is in [GHKS, Section 10.4], see also [GHKS, Remark 10]. The authors use the terminology optimal glueing in this context. The proof given there takes the work of [FS86] as a starting point, but a more detailed analysis of the period map is required. The uniqueness result also follows from [AE21, Proposition 5.12]. We also note that the uniqueness of $Y$ is implicitly contained in [Laz08, Remark 5.5].

Remark 2.12. We will now consider deformations $\mathcal{Y} \rightarrow S$ of $d$-semistable K3 surfaces. The relevant relative notions of divisors and cones are recalled in Section 3. Here we simply remark that under our assumptions on $S$, linear equivalence over the base coincides with the usual linear equivalence, in particular $\operatorname{Pic}(\mathcal{Y} / S)=\operatorname{Pic}(\mathcal{Y})$

The following will play an important role for us.

Proposition 2.13. Let $Y$ be a projective type III d-semistable K3 surface with $c_{Y}=1$. Then there is a unique (up to automorphism of the base $S)$ Kulikov model $\mathcal{Y} \rightarrow S$ of $Y$ such that

$$
r_{c}: \operatorname{Pic}(\mathcal{Y} / S) \cong \operatorname{Pic}(Y)
$$


with $r_{c}$ being the restriction map. Also, if $k$ is the index and $t$ is the number of triple points of $Y$ then

$$
\operatorname{Pic}\left(\mathcal{Y}_{\eta}\right) \cong 2 E_{8}(-1) \oplus U \oplus\left\langle\frac{-t}{k^{2}}\right\rangle .
$$

Proof. This goes back to [FS86]. If $c_{Y}=1$, then $\operatorname{Pic}(Y) \cong L$. The surface $Y$ also determines the invariants $t$ and $k$. Let $n$ be the number of components of $Y$. Consider the divisors $\xi_{i}=\sum_{j} D_{i j}-D_{j i}, i=1, \ldots, n$ defined above. Note that $\sum_{i} \xi_{i}=0$. The lattice $\left\langle\xi_{1}, \ldots \xi_{n} \mid \sum_{i} \xi_{i}=0\right\rangle$ is a primitive sublattice of $L$ by [FS86, (4.13)]. Hence we can pick linearly independent divisors $L_{1}, \ldots, L_{19}$ that generate $\operatorname{Pic}(Y) \bmod K$. If $\mathcal{Y} \rightarrow S$ is a deformation with $\operatorname{Pic}(\mathcal{Y} / S) \cong \operatorname{Pic}(Y)$ via restriction, then by definition $\mathcal{Y} \rightarrow S$ is a deformation of the tuple $\left(Y ; L_{1}, \ldots, L_{19}\right)$. We shall show that there is a unique such 1-parameter deformation.

Let $X \rightarrow V$ be the semiuniversal analytic deformation of $Y$ as defined in the proof of [Fri83, Theorem 5.10]. By the arguments in the proof of [FS86, Lemma 5.5], the locus $V^{\prime}$ in the smoothing component of $V$ where the $L_{i}$ deform is 1-dimensional and smooth. Let $\mathfrak{X}^{\prime} \rightarrow V^{\prime}$ be the restriction of the semiuniversal family. By [Fri83, Theorem 5.10], this is a smoothing of $Y$. Let $W^{\prime}$ be the analytic algebra defining the germ $V^{\prime}$, and let $W$ be the completion of $W^{\prime}$ with respect to the maximal ideal. Then $W \cong \mathbb{C}[[t]]$. This defines a formal scheme $\hat{\mathcal{Y}} \rightarrow \operatorname{Spf} \mathbb{C}[[t]]$, and by the condition that all $L_{i}$ deform, there is an $\mathcal{L} \in \operatorname{Pic}(\hat{\mathcal{Y}})$ restricting to an ample line bundle on $Y$ and thus by Grothendieck's existence theorem a deformation $\mathcal{Y} \rightarrow S$ with $S=\operatorname{Spec} \mathbb{C}[[t]]$ such that $\hat{\mathcal{Y}}$ is the completion of $\mathcal{Y} \rightarrow S$ along $Y$. By construction, $\operatorname{Pic}(\mathcal{Y} / S) \cong \operatorname{Pic}(Y)$ via restriction.

We show that $\mathcal{Y} \rightarrow S$ is a smoothing of $Y$. The total space of the deformation $\mathcal{X}^{\prime} \rightarrow V^{\prime}$ is smooth, as follows from [Fri83, Theorem 5.10]. In particular, its local rings in closed points are regular, and thus by [Mat89, Theorem 23.7] the local rings $\mathcal{O}_{\hat{\mathcal{y}}, y}$ for $y \in Y$ of the formal smoothing are also regular. By the same theorem, this implies that the stalks of $\mathcal{O}_{\mathcal{Y}, y}$ at closed points of the central fibre of $\mathcal{Y} \rightarrow S$ are regular local rings. This implies that $\mathcal{Y}$ is regular by [GW10, Remark 6.25]. In particular, the generic fibre is a smooth K3 surface. Also, by adjunction, $\mathcal{Y}$ has trivial canonical bundle. So $\mathcal{Y} \rightarrow S$ is indeed a semistable model.

Also, for the degeneration $\mathcal{Y} \rightarrow S$, it follows from [Kaw97, Lemma 4.2], using the fact that $S$ is a $D V R$, that we have an exact sequence

$$
0 \rightarrow \mathbb{Z}^{\mathcal{Y}} \rightarrow \operatorname{Pic}(\mathcal{Y} / S) \rightarrow \operatorname{Pic}\left(\mathcal{Y}_{\eta}\right) \rightarrow 0
$$

with $\mathbb{Z}^{\mathcal{Y}}$ the abelian group generated by the components $Y_{i}$ of the central fibre modulo the relation $\sum_{i} Y_{i}=0$. The statement about the Picard group of the generic fibre then follows from [Laz08, Prop. 4.3] and [Laz08, Corollary 4.6] together with Sequence 2.6. So $\mathcal{Y} \rightarrow S$ is a model as claimed.

Now, suppose $\mathcal{Y}^{\prime} \rightarrow S$ is a second model. By formal semiuniversality, $\mathcal{Y}^{\prime} \rightarrow S$ is pulled back from $\mathcal{Y} \rightarrow S$ via a homomorphism $\mathbb{C}[[t]] \rightarrow \mathbb{C}[[t]]$. Because $\mathcal{Y}^{\prime} \rightarrow S$ is regular, the uniformizing parameter $t$ maps to at with $a$ a unit. Hence $\mathcal{Y}^{\prime} \rightarrow S$ is canonically isomorphic to $\mathcal{Y} \rightarrow S$. This proves the result.

Remark 2.14. In this section we allow automorphisms which lie over an automorphism of the base $S$ given by sending the uniformizing parameter $t$ to $a t$, with $a$ a unit. From Section 3 onwards we will choose a fixed reference model and then work over a fixed base $S$. The final result will be independent of the choice of this reference model.

Definition 2.15. We shall call a $d$-semistable $\mathrm{K} 3$ surface $Y$ with $c_{Y}=1$ maximal and a degeneration $\mathcal{Y} \rightarrow S$ of a maximal $\mathrm{K} 3$ surface with $r_{c}$ : $\operatorname{Pic}(\mathcal{Y} / S) \cong \operatorname{Pic}(Y)$ a maximal degeneration.

The next proposition says that maximal degenerations behave well under flops.

Proposition 2.16. Let $\mathcal{Y} \rightarrow S$ be a maximal degeneration with central fibre $Y=\mathcal{Y}_{c}$ and let $\mathcal{Y}^{+} \rightarrow S$ be a flop of $\mathcal{Y} \rightarrow S$. Then $\mathcal{Y}^{+} \rightarrow S$ is again a maximal degeneration and the dual graph of the central fibre $Y^{+}=\mathcal{Y}_{c}^{+}$is a triangulation of $S^{2}$ with the same number of triangles as the dual graph of $Y$. 
Proof. We shall prove this result using the analytic theory. For this we first note that any flop factors into flops given by contractions of extremal rays, see [KM98, §6.4]. Thus we can assume the flop is given by a small contraction $\operatorname{contr}_{R}$ with $R$ an extremal ray of $\mathcal{Y} \rightarrow S$. Let $\pi: \mathcal{Y} \rightarrow \overline{\mathcal{Y}}$ be the flopping contraction over $S, F$ be a divisor that is anti-ample on the fibres of $\pi$. The morphism $\pi$ is given by a divisor $G$ on $\mathcal{Y}$ with restriction $G_{c}$ to $Y$. This defines a divisor $G^{\prime}$ on the maximal analytic smoothing $\mathcal{X} \rightarrow \mathbb{D}$ of $Y$ and a contraction $\mathcal{X} \rightarrow \overline{\mathcal{X}}$ of the extremal ray $R$. Hence $\mathcal{X} \rightarrow \overline{\mathcal{X}}$ is a small contraction. Restricting the divisor $F$ to the central fibre and extending by maximality to $\mathcal{X}$ we obtain the induced divisor $F^{\prime}$ on $\mathcal{X}$. This is anti-ample on the fibres of $\mathcal{X} \rightarrow \overline{\mathcal{X}}$. Hence there is a flop $\mathcal{X}^{+} \rightarrow \overline{\mathcal{X}}$. By a result of Kulikov, $\mathcal{X}^{+} \rightarrow \mathbb{D}$ is semistable, see e.g. [Cor95, Corollary 3.7]. Completion along the central fibre gives morphisms of the corresponding formal schemes, and by Grothendieck's existence theorem, we get proper morphisms $f: \mathcal{Y} \rightarrow \mathcal{Z}$ and $f^{+}: \mathcal{Y}^{+} \rightarrow \mathcal{Z}$. We claim that the morphism $f: \mathcal{Y} \rightarrow \mathcal{Z}$ is given by the contraction of $R$. Indeed, we have $f_{*} \mathcal{O}_{\mathcal{Y}} \cong \mathcal{O}_{\mathcal{Z}}$ : the restriction to the central fibres $f_{c}: \mathcal{Y}_{c} \rightarrow \mathcal{Z}_{c}$ is a proper surjective birational morphism with connected fibres. It is is straightforward to check that $\left(f_{c}\right)_{*} \mathcal{O}_{Y} \cong \mathcal{O}_{Z}$, using that every regular section of $\mathcal{O}_{\mathcal{Y}}$ is constant on the fibres. By the global version of [Wah76, Lemma 1.2], we have $\left(f_{n}\right)_{*} \mathcal{O}_{\mathcal{Y}_{n}} \cong \mathcal{O}_{\mathcal{Z}_{n}}$ for the truncations of order $n$ and thus $\hat{f}_{*} \widehat{\mathcal{O}_{\mathcal{Y}}} \cong \widehat{\mathcal{O}_{\mathcal{Z}}}$, by [IIl05, Theorem 8.2.2]. By the same theorem, $\hat{f_{*}} \widehat{\mathcal{O}_{\mathcal{Y}}} \cong \widehat{f_{*} \mathcal{O}_{\mathcal{Y}}}$, so $\widehat{\mathcal{O}_{\mathcal{Z}}} \cong \widehat{f_{*} \mathcal{O}_{\mathcal{Y}}}$ and from [IIl05, Theorem 8.4.2], it follows that $f_{*} \mathcal{O}_{\mathcal{Y}} \cong \mathcal{O}_{\mathcal{Z}}$. As $\mathcal{Z}$ is a Nagata scheme, its normalisation is finite over $\mathcal{Z}$. Because of the universal property of normalisation and connectedness of the fibres of $f$, it follows from finiteness that $\mathcal{Z}$ is normal. By uniqueness of contractions, $f=\pi$ and, in particular $\overline{\mathcal{Y}}=\mathcal{Z}$.

Hence $f: \mathcal{Y}^{+} \rightarrow \mathcal{Z}$ is the flop of $\pi$. It has central fibre $\mathcal{Y}_{c}^{+}=Y^{+} \cong\left(\mathcal{X}^{+}\right)_{c}$, which is a $d$-semistable K3 surface of type III. As $Y^{+}$is the central fibre of a degeneration, it follows that the $\xi_{i}$ classes from above are in fact Cartier. Since we have a type III degeneration, the dual graph is a triangulation of the sphere $S^{2}$ by Kulikov's theorem [Kul77, Theorem II]. This has the same number $n$ of triangles as the dual graph of $Y$, because the number of components is the same, and hence also the same number of triple points $t$, since $t=2 n-4$, see e.g. [Laz08, 3.2]. Also, $\mathcal{Y} \rightarrow S$ and $\mathcal{Y}^{+} \rightarrow S$ have the same primitivity $k$, as can be seen from the monodromy. Hence the lattices given by $L$ and $L^{+}$modulo the saturation of the sublattice spanned by the $\xi_{i}$ are both isomorphic to $2 E_{8}(-1) \oplus U \oplus\left\langle-2 t / k^{2}\right\rangle$. Since moreover $\operatorname{Pic}\left(\mathcal{Y}_{\eta}\right) \cong \operatorname{Pic}\left(\mathcal{Y}_{\eta}^{+}\right) \cong 2 E_{8}(-1) \oplus U \oplus\left\langle-2 t / k^{2}\right\rangle$ it follows that the inclusion given by restriction to the central fibre $\mathcal{Y}_{c}$ defines in fact isomorphisms $\operatorname{Pic}\left(\mathcal{Y}^{+}\right) \cong \operatorname{Pic}\left(\mathcal{Y}_{c}^{+}\right) \cong L^{+}$and hence $\mathcal{Y}^{+} \rightarrow S$ is also maximal.

We shall now consider the special case arising from the mirror families of $2 d$-polarized $K 3$ surfaces. We will also construct explicit models. Here we first state the more general

Proposition 2.17. Let $d>0$ and $\check{M}_{2 d}=U \oplus 2 E_{8}(-1) \oplus\langle-2 d\rangle$. Then there exists a primitive maximal Kulikov model $\mathcal{Y} \rightarrow S$ such that $\operatorname{Pic}\left(\mathcal{Y}_{\eta}\right) \cong \check{M}_{2 d}$. Any two such Kulikov models are related by a sequence of flops.

Proof. The existence follows from Proposition 2.13, all we require is the existence of a primitive type III $d$-semistable K3 surface with $t=2 d$ triple points and primitivity $k=1$, which exists by [FS86, Theorem 0.6]. We now show that two such models are related by flops. For this let $\mathcal{Y} \rightarrow S$ and $\mathcal{Y}^{\prime} \rightarrow S$ be two distinct deformations with the properties stated and central fibres $Y$ and $Y^{\prime}$ respectively. It follows from Sequence (2.6) and e.g. [Laz08, Proposition 4.3] that both central fibres have exactly $2 d$ triple points. Also, both degenerations are primitive. As above, we have the maximal analytic family $\mathcal{X} \rightarrow \mathbb{D}$ over a small disc with central fibre $Y, \operatorname{Pic}(\mathcal{X}) \cong \operatorname{Pic}(Y)=L$ and smooth fibres K3 surfaces with Picard rank 19. Similarly we have $\mathcal{X}^{\prime} \rightarrow \mathbb{D}$ with central fibre $Y^{\prime}$.

By [FS86, Theorem 0.6] there is a sequence of type I and type II modifications $\mathcal{X} \rightarrow \mathcal{X}^{\prime}$. As both models are projective, this sequence factors into a sequence of projective flops given by contractions of extremal rays, by [KM98, Remark 6.37]. Let $F$ be an effective divisor defining the first flop, say $\mathcal{X} \rightarrow \mathcal{X}^{+}$in this sequence. Restriction to the central fibre and then lifting to $\mathcal{Y} \rightarrow S$ via maximality yields an effective divisor $F^{\prime}$ inducing a contraction of an extremal ray. As the exceptional locus on $Y$ does not deform to $\mathcal{Y}$ (because it does not deform on $\mathcal{X}$, so it does not fullfill the numerical conditions for lifting to the family), we obtain a 
flop $\mathcal{Y}^{+}$of $\mathcal{Y}$ with $\mathcal{Y}_{c}^{+}=\mathcal{X}_{c}^{+}$. Proceeding in this way, one obtains a sequence of flops

$$
\mathcal{Y} \rightarrow \cdots \rightarrow \mathcal{Y}^{\prime \prime}
$$

such that the central fibre of $\mathcal{Y}^{\prime \prime}$ is $Y^{\prime}$. By uniqueness of maximal smoothings, see Proposition 2.13, $\mathcal{Y}^{\prime} \cong \mathcal{Y}^{\prime \prime}$.

Remark 2.18. For the sake of completeness we would like to point out that there are no type 0 flops between maximal Kulikov models. The reason is the following. Such a flop requires a rigid (-2)-curve on the central fibre, i.e. a curve with normal bundle $\mathcal{O}(-1) \oplus \mathcal{O}(-1)$. Since, however, every smooth curve in the interior of a component of the central fibre, can, by maximality, be extended to the general fibre, such a curve does not exist.

Remark 2.19. Proposition 2.17 shows that the generic fibre $\mathcal{Y}_{\eta}$ of a maximal primitive degeneration $\mathcal{Y} \rightarrow S$ with $\operatorname{Pic}\left(\mathcal{Y}_{\eta}\right)=\check{M}_{2 d}$ is independent of $\mathcal{Y} \rightarrow S$.

This allows us to make the following definition, where we follow the established terminology in the existing literature:

Definition 2.20. Fix $2 d>0$ with $d$ square free. The (unique) $K 3$ surface $\mathcal{Y}_{\eta} \rightarrow \operatorname{Spec}(\mathbb{C}((t)))$ with $\operatorname{Pic}\left(\mathcal{Y}_{\eta}\right)=\check{M}_{2 d}$ which is the generic fibre of some maximal primitive smoothing $\mathcal{Y} \rightarrow S$ is called the Dolgachev-Nikulin-Voisin family of degree $2 d$.

Remark 2.21. Here, we require $d$ to be square free to obtain the Dolgachev-Nikulin-Voisin family as in [GHKS]. For $d$ not square free, there are several distinct local models.

Definition 2.22. We fix $2 d>0$ with $d$ square free. Then any primitive projective type III degeneration $\mathcal{Y} \rightarrow S$ that is maximal and whose generic fibre has Picard group $\check{M}_{2 d}$ is called a model of the DolgachevNikulin-Voisin family of degree $2 d$.

We will abbreviate Dolgachev-Nikulin-Voisin family by DNV family. We note the following result.

Proposition 2.23. Let $Y$ be a maximal projective d-semistable K3 surface. Then there exists a sequence of elementary modifications of type I and II

$$
Y \rightarrow Y_{1} \rightarrow \cdots \rightarrow Y_{i} \rightarrow \cdots \rightarrow Y_{n}
$$

such that all $Y_{i}$ are maximal d-semistable $K 3$ surfaces and $Y_{n}$ is in $(-1)$-form.

Proof. Let $\mathcal{X} \rightarrow \mathbb{D}$ be the maximal analytic smoothing of $Y$. By the (-1)-Theorem, see Theorem 2.7, there is a sequence of type I and type II modifications

$$
\mathcal{X}=\mathcal{X}_{0} \rightarrow \mathcal{X}_{1} \rightarrow \cdots \rightarrow \mathcal{X}_{i} \rightarrow \cdots \rightarrow \mathcal{X}_{n}
$$

such that the central fibre of $\mathcal{X}_{n}$ is in $(-1)$-form. In this sequence the lattices $L\left(\mathcal{X}_{i}\right)$ and $L\left(\mathcal{X}_{i+1}\right)$ are canonically identified, and the Carlson map pulls back to the Carlson map, cf. [AE21, Proposition 5.18]. This shows that maximality is preserved (as all of $L$ is the kernel of the Carlson map). Thus, restricting the sequence (2.7) to the central fibre gives the result.

\subsection{Construction of Models}

There is a bijection between triangulations $\mathscr{G}$ of the sphere such that no vertex has valency greater than 6 and locally trivial deformation classes $[Y]_{\mathscr{G}}$ of d-semistable $\mathrm{K} 3$ surfaces of type III in $(-1)$-form, see [Laz08, §5.1]. By Lemma 2.11, there is a unique element $Y_{\mathscr{G}} \in[Y]_{\mathscr{G}}$ with trivial Carlson extension. Here we describe the components of these surfaces and their gluing. Each vertex $v$ of $\mathscr{G}$ corresponds to a component whose normalisation is a very special (weak) del Pezzo surface of degree the valency of $v$ for which we give an 

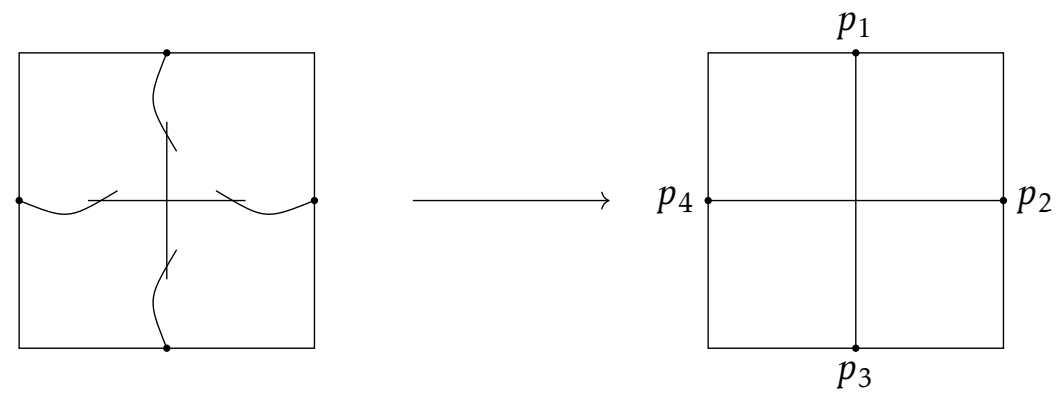

Figure 4. The $(1,1,1,1)$ - blow-up of $\mathbb{P}^{1} \times \mathbb{P}^{1}$ in $\left(p_{1}, p_{2}, p_{3}, p_{4}\right)$.

explicit geometric construction below. The edges of $\mathscr{G}$ then determine which components are glued. We emphasize that this construction is at least implicit in [GHKS].

We first make the following definition.

Definition 2.24. Let $(Y, D)$ be an anticanonical pair. Let $D=\sum D_{i}$ and $p$ be a smooth point of $D$ lying on the component $D_{i}$. If $n=1$, the $n$-fold blow-up of $Y$ in $p$ is the usual blow-up, if $n>1$, the n-fold blow-up of $Y$ in $p$ is the blow-up of the $n-1$-fold blow-up $\pi: Y^{\prime} \rightarrow Y$ in the point $\operatorname{ex}(\pi) \cap D_{i}^{\prime}$, where $D_{i}^{\prime}$ is the strict transform of $D_{i}$ on $Y^{\prime}$. More generally, if $\left(p_{1}, \ldots, p_{k}\right)$ is an ordered set of points $p_{j} \in Y$, which lie on the smooth locus of $D$ such that each $D_{i}$ contains at most one point $p_{j}$, we define by the obvious generalisation the $\left(n_{1}, \ldots, n_{k}\right)$-blow-up of $Y$ in $\left(p_{1}, \ldots, p_{k}\right)$.

Construction 2.25. Here we describe and construct the possible components of the unique elements $Y_{\mathscr{G}} \in[Y]_{\mathscr{G}}$ in $(-1)$-form with trivial Carlson extension. The normalisations of these components are (weak) del Pezzo surfaces of degree $d=1, \ldots, 6$ and the double locus of the central fibre gives rise to an anticanonical cycle $D$ on the normalisation $Y$ of such a component. The geometry of the pairs $(Y, D)$ is discussed in detail in [Laz08, §5.1], see in particular [Laz08, Proposition 5.2] and [Laz08, Lemma 5.14]. The first property is that $D$ is a cycle of $(-1)$-curves if $d>1$ or $D^{2}=1$ if $d=1$ respectively. In degree $d=5,6$ this leads to a unique pair $(Y, D)$. For $d=1,2,3$ and 4 it follows from [Laz08, Proposition 5.2] that the orthogonal complement of the sublattice spanned by the components of the effective anti-canonical cycle $D$ in the Picard group is a root lattice of type $E_{8}, E_{6}, D_{4}$ or $A_{2}$ respectively. The (weak) del Pezzo surfaces which we need here are the ones which Laza calls maximally algebraic, see [Laz08, Remark 5.5]. The relevant condition is that the root lattices $E_{8}, E_{6}, D_{4}$ or $A_{2}$ respectively, are generated by effective $(-2)$ curves. This condition will be needed to obtain that the Carlson map $c_{Y_{\mathscr{G}}}=1$. Contracting these (-2)-curves gives rigid singular del Pezzo surfaces which are uniquely determined, see [Laz08, Lemma 5.14]. Below we will construct these surfaces explicitly. We will further specify special points on each component of the anticanonical cycle $D$. We will call a special point interior if it is not a node of $D$. The special points are used to define the gluing of the components to finally obtain the unique element $Y_{\mathscr{G}}$ in the trivial deformation class $[Y]_{\mathscr{G}}$.

$d=1$ : Let $Q=\mathbb{P}^{1} \times \mathbb{P}^{1}$ with toric boundary $\tilde{D}=\tilde{D}_{1}+\tilde{D}_{2}+\tilde{D}_{3}+\tilde{D}_{4}$, ordered cyclically. Let $p_{i} \in \tilde{D}_{i}$, $i=1, \ldots, 4$, be points in the smooth part of $\tilde{D}$ such that $p_{i}, p_{i+2}$ are in the same fibre of one of the two rulings, see the right hand side of Figure 4 . Let $\tilde{Q}$ be the $(1,5,1,3)$-blow-up of $Q$ in $\left(p_{1}, p_{2}, p_{3}, p_{4}\right)$. The strict transforms of $\tilde{D}_{1}, \tilde{D}_{3}$ have self-intersection -1 on $\tilde{Q}$. Blowing down these yields a surface such that the strict transform of $\tilde{D}_{4}$ has self intersection $(-1)$. Then, blowing this down gives a surface $\bigcup_{1}$ with an anticanonical cycle $D$ of self intersection 1 and an $E_{8}$ root system of effective (-2)-curves. This is a weak del Pezzo of degree 1 , see Figure 5 . There is a unique $(-1)$-curve $E$ meeting $D$. The special points are the node of $D$ and the point $E \cap D$ (which is an interior special point).

$d=2$ : For degree 2 , we take $\mathbb{P}^{2}$ together with its toric boundary $(x y z=0)$. We can fix three collinear points, one on each boundary divisor, say $p, q, r$. Let $Y^{\prime}$ be the $(3,3,2)$-blow-up of $\mathbb{P}^{2}$ in $(p, q, r)$. This yields a weak del Pezzo surface of degree 1, as we have blown up 8 points that are not on $(-2)$ curves. 


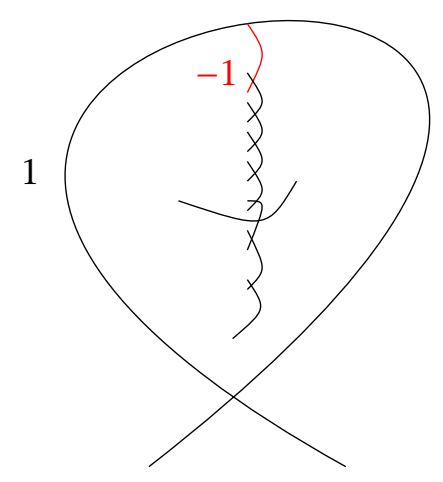

Figure 5. The surface $\bigsqcup_{1}$.

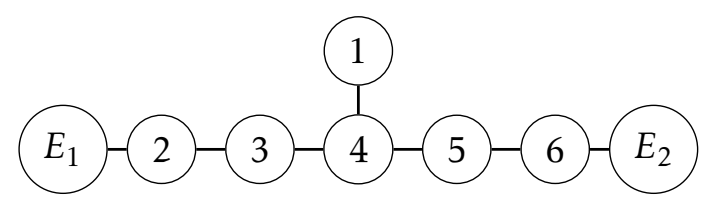

Figure 6. The $E_{6}$ root system and the exceptional curves $E_{1}, E_{2}$.

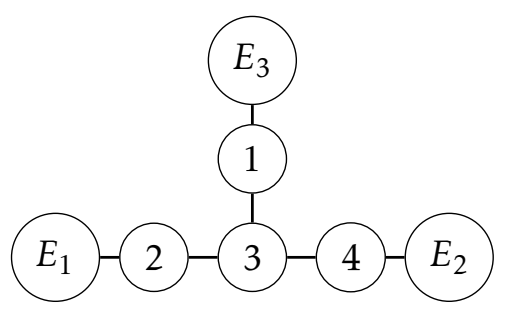

Figure 7. The $D_{4}$ root system and the exceptional curves $E_{1}, E_{2}, E_{3}$.

Now, blow down the strict transform of the toric divisor that is a $(-1)$-curve. The resulting surface $\bigcup_{2}$ is a weak del Pezzo surface of degree 2 with anticanonical cycle $D=D_{1}+D_{2}$. It carries an $E_{6}$ configuration of effective $(-2)$-curves by construction. There are also 2 exceptional curves $E_{1}, E_{2}$ of the first kind each meeting a long end of the root system and a component of the anticanonical divisor, see Figure 6. The special points of $D_{i}$ are the points $D_{i} \cap E_{i}$ and the two points of the intersection $D_{1} \cap D_{2}$.

$d=3$ : For degree 3 , we take again $\mathbb{P}^{2}$ together with its toric boundary $(x y z=0)$. As above, we fix three collinear points, one on each boundary divisor, say $p, q, r$. Let $Y$ be the $(2,2,2)$-blow-up of $\mathbb{P}^{2}$ in $(p, q, r)$, a weak del Pezzo surface of degree 3. It carries a $D_{4}$ root system of effective $(-2)$-curves, given by the strict transform of the line through the points $p, q, r$ together with the strict transforms of the first blow-ups in each point. The anticanonical cycle $D=D_{1}+D_{2}+D_{3}$ is given by the strict transform of the toric boundary. There are also three irreducible $(-1)$-curves $E_{i}$ in the exceptional locus of the blow-up, each meeting a unique component $D_{i}$. The special points are again the nodes of the anticanonical cycle and the points $E_{i} \cap D_{i}$, see Figure 7.

$d=4$ : Again, let $Q=\mathbb{P}^{1} \times \mathbb{P}^{1}$ with toric boundary $\tilde{D}=\tilde{D}_{1}+\tilde{D}_{2}+\tilde{D}_{3}+\tilde{D}_{4}$. Let $p_{i}, i=1, \ldots, 4$, be points on the intersection of the fibres of the two ruling with the toric boundary components. Blow up $Q$ once in each $p_{i}$, the resulting surface $\bigcup_{4}$ is a weak del Pezzo of degree 4 , with an $A_{2}$ root system of effective (-2)-curves and an anticanonical cycle $D=D_{1}+D_{2}+D_{3}+D_{4}$ where the $D_{i}$ are the strict transforms of the $\tilde{D}_{i}$. There are $4(-1)$-curves $E_{i}, i=1, \ldots, 4$ on $\bigcup_{4}$ that are not components of $D$, 
each meeting exactly one of the $D_{i}$ transversally. The special points of $D$ are the points $D_{i} \cap E_{i}$ and $D_{i} \cap D_{i+1}$, where the indices are considered cyclically, see Figure 4 .

$d=5$ : Pick 4 points $\{p, q, r, s\}$ in $\mathbb{P}^{2}$, no three on a line. Then $\mathrm{Bl}_{\{p, q, r, s\}}\left(\mathbb{P}^{2}\right)$ is the del Pezzo surface of degree 5. The anticanonical divisor $D=D_{1}+D_{2}+D_{3}+D_{4}+D_{5}$ is a cycle of $(-1)$-curves forming a pentagon, which we obtain as follows: we choose the strict transforms of the lines spanned by $(p, q),(p, r)$ and $(q, s)$, together with the exceptional lines which arise form blowing up $p$ and $q$. Note that permutations of the points $p, q, r, s$ gives rise to isomorphic pairs $(Y, D)$. There are 5 more $(-1)$-curves $E_{i}, i=1, \ldots, 5$. Each $E_{i}$ meets exactly one of the $D_{i}$ transversally. The special points of $D_{i}$ are the points $D_{i} \cap E_{i}$ and $D_{i} \cap D_{i+1}$.

$d=6$ : Pick the three coordinate points $\{p, q, r\}$ in $\mathbb{P}^{2}$ which are torus orbits. Then $\bigsqcup_{6}=\mathrm{Bl}_{\{p, q, r\}}\left(\mathbb{P}^{2}\right)$ is the del Pezzo surface of degree 6. Let $D=D_{1}+D_{2}+D_{3}+D_{4}+D_{5}+D_{6}$ be the anticanonical divisor which consists of the strict transforms of the coordinate lines and the exceptional lines. This is a cycle of $(-1)$-curves forming a hexagon. The toric structure of $\bigcup_{6}$ indentifies a copy of $\mathbb{G}_{m} \subset D_{i}$ for each $i$. The special points of $D_{i}$ are the points $-1 \in \mathbb{G}_{m}$, and $D_{i} \cap D_{i+1}$.

Definition 2.26. We will denote the (weak) del Pezzo surfaces of degree $d$ constructed here by $\bigcup_{d}$.

Now, the triangulation $\mathscr{G}$ defines a locally trivial deformation class $[Y]_{\mathscr{G}}$ of $d$-semistable $\mathrm{K} 3$ surfaces and we can take the member $Y_{\mathscr{G}}$ of $[Y]_{\mathscr{G}}$ such that the (normalisation) of each component is isomorphic (as an anticanonical pair) to a surface from the above list, by [Laz08, Lemma 5.14], where we recall that the valency of a vertex is equal to the degree of the corresponding (weak) del Pezzo surface. The gluing is such that the special points are identified pairwise where, in particular, interior special points are identified with interior special points.

We learned the next result from [GHKS]:

Proposition 2.27. Let $\mathscr{G}$ be a triangulation with valency at most 6 and no $k$-bands of hexagons. Then the surfaces $Y_{\mathscr{G}}$ are maximal, i.e. have trivial Carlson map $c_{Y_{\mathscr{G}}}=1$.

Proof. A proof can be found in [GHS16, Construction 10.14]. Here we sketch a proof in the case of simple normal crossing. Each of the surfaces $\bigcup_{i}$, for $i=1 \ldots 5$, has a $\mathbb{Q}$-basis $B_{i}$ of $\operatorname{Pic}\left(\bigcup_{i}\right)$ given by the $(-2)$-curves and the interior $(-1)$-curves, i.e. those that are not components of the anticanonical divisor. Let $Y_{\mathscr{G}}=\cup_{j \in J} Y_{j}$. We shall choose an order on $J$. The Picard group of $Y_{\mathscr{G}}$ is given by the kernel of the Carlson map. Let $Y_{\text {simp }}$ be a semi-simplicial resolution of $Y_{\mathscr{G}}$, see [Car80], [KK98, §4.2.2]. Then one defines $Y_{p}$ to be

$$
Y_{p}=\coprod Y_{j_{0}} \cap \cdots \cap Y_{j_{p}} \quad\left(j_{0}<\cdots<j_{p}\right)
$$

for $p=0,1,2$. Face maps are given by maps $\delta_{p}: Y_{p} \rightarrow Y_{p-1}$ such that $\delta_{p}$ is the inclusion on the components of $Y_{p}$, see [KK98, §4.2.2]. We start with a collection of line bundles $L_{j}, j \in J$ on the components $Y_{j}$ such that the degrees on the double curves $D_{i j}$ coincide. We want to show that these glue to a line bundle $L$ on $Y_{\mathscr{G}}$. For $i(j) \leq 5$ we write $L_{j}$ in the basis $B_{i(j)}$. Also, given a line bundle $L_{j}$ on a hexagonal component $\bigcup_{6}$, we can find a linearly equivalent divisor on $Y_{j}$ whose restriction to a component $D_{k}$ of the anticanonical divisor of $\bigcup_{6}$ is $\left(\operatorname{deg} L_{j \mid D_{k}}\right) p_{k}$ where $p_{k}$ denotes the interior special point on $D_{k}$. This follows from (the proof of) [GHK15, Lemma 2.8]. The line bundles $L_{j}$ can therefore be represented by divisors whose restrictions to the double curves coincide. It follows that the collection $L=\left\{L_{j}, j \in J\right\}$ is in the kernel of the Carlson map as follows from [Car80, p. $277 \mathrm{ff}]$ applied to $Y_{\text {simp. This implies the result. }}$

We also obtain that maximal $d$-semistable $K 3$ surfaces in (-1)-form are always projective.

Proposition 2.28. Let $Y=\cup Y_{i}$ be a d-semistable $K 3$ surface of type III with $c_{Y}=1$. If $Y$ is in (-1)-form, then $Y$ is projective. 
Proof. Let $Y$ be a surface as in the proposition. As we have discussed above it follows from the work of Laza [Laz08, §5.1] that the normalisations $Y_{i}^{v}$ of the components $Y_{i}$ of $Y$ are weak del Pezzo surfaces of degree $d_{i} \leq 6$ and that the orthogonal complement of the lattice spanned by the components of the anitcanonical cycle $D=\sum D_{i}$ on $Y_{i}^{v}$ in $\operatorname{Pic}\left(Y_{i}\right)$ is a root lattice $E_{8}, E_{6}, D_{4}$ or $A_{2}$ for $d_{i}=1,2,3$ and 4 , respectively and empty for $d_{i}=5,6$. Moreover we know that these root lattices are generated by effective $(-2)$-curves. We show that $Y$ is projective by giving an ample bundle $A_{i}$ on each $Y_{i}^{v}$ such that $A_{i} \cdot D_{i j}=A_{i} \cdot D_{i k}$ for all $j, k$, i.e. such that the $A_{i}$ have the same degree on all components of the anticanonical cycle on $Y_{i}^{v}$. By Lemma 2.10, after taking suitable multiples, one can then glue these line bundles to obtain a bundle $A$ on $Y$. The bundle $A$ is ample as its restriction to each irreducible component is ample, by [Laz04, Proposition 1.2.16]. As the structure morphism is proper, $\mathrm{Y}$ is projective. To simplify notation, we taciturnly assume $Y_{i}^{v}=Y_{i}$. For $d_{i}=1$ there is nothing to show. For $d_{i}=5,6$ the claim is obvious, we can take the anticanonical divisor. We consider the cases $d_{i}=2,3$. For the root lattices $R_{i}$ described above we have a natural root basis given by the set $B\left(Y_{i}\right)$ of $(-2)$-curves constructed in the blow-up procedure. Note also that for all such $Y_{i}$ by construction we have $(-1)$-curves as in Figures 6 and 7 connecting the root system to the boundary. We denote the set of these curves by $E\left(Y_{i}\right)$.

Suppose there is an integer $e>0$ and a divisor

$$
A_{i}=\sum_{B_{j} \in B\left(Y_{i}\right)} b_{j} B_{j}+\sum_{E_{k} \in E\left(Y_{i}\right)} e E_{k}
$$

in $\operatorname{Pic}\left(Y_{i}\right)$ such that $A_{i} \cdot C>0$ for all $C \in B\left(Y_{i}\right) \cup E\left(Y_{i}\right)$. Then $A_{i}$ defines an ample bundle on $Y_{i}$ : being a (weak) del Pezzo surface, $Y_{i}$ is a Mori Dream space by [TVV11, Theorem 2.9], so in particular, the cone of curves of $Y_{i}$ is rational polyhedral. The Picard rank is greater than 3 and thus, by [AL11, Proposition 1], $\operatorname{Pic}\left(Y_{i}\right)$ generated by curves $C$ with $C^{2}<0$, i.e. by $(-2)$ and $(-1)$-curves. Let $C$ be such a curve. If $C \in B\left(Y_{i}\right) \cup E\left(Y_{i}\right)$, then $A_{i} \cdot C>0$ by assumption. If $C$ is not in $B\left(Y_{i}\right) \cup E\left(Y_{i}\right), C$ is a component of $D$, as follows from Proposition 4.5 below (which is independent of this result). Then $C \cdot D=e$. Hence $A_{i}$ is strictly positive on the (polyhedral) cone of curves, and hence ample by Kleiman's criterion. We define such $A_{i}$ 's as follows: for $E_{6}$, take the numbers $(7,11,13,16,13,11)$ for the roots, where the number in the $i$-th position is the coefficient of the $i$-th root, with indexing as in the figures, and set $e=10$. For $D_{4}$ we can take $(11,11,13,11)$ and $e=10$.

It remains to consider the case $d_{i}=4$. In this case $Y_{4}=\bigcup_{4}$ with the $A_{2}$ root system and the same construction as before with numbers $(3,3)$ on the root system and $e=2$ defines an ample bundle $A_{i}$.

We have the following proposition.

Proposition 2.29. Let $\mathscr{G}$ be a triangulation of $S^{2}$ with $2 d$ triangles with valency at most 6 and without $k$-bands of hexagons for any $k>1$.

The surface $Y_{\mathscr{G}}$ is a maximal primitive d-semistable K3 surface. The associated maximal smoothing $\mathcal{Y}_{\mathscr{G}} \rightarrow S$ is a model in (-1)-form of the Dolgachev-Nikulin-Voisin family of degree $2 d$, i.e. $\operatorname{Pic}\left(\mathcal{Y}_{\mathscr{G}_{\eta}}\right)=\check{M}_{2 d}$ and $\operatorname{Pic}\left(\mathcal{Y}_{\mathscr{G}} / S\right) \cong \operatorname{Pic}\left(Y_{\mathscr{G}}\right)$ by restriction. The threefold $\mathcal{Y}_{\mathscr{G}}$ is Calabi-Yau with $H^{1}\left(\mathcal{Y}_{\mathscr{G}}, \mathcal{O}_{\mathcal{Y}_{\mathscr{G}}}\right)=0$ and projective over the base $S$.

Proof. $Y_{\mathscr{G}}$ is a $d$-semistable $\mathrm{K} 3$ surface in $(-1)$-form by construction. It is primitive because the dual graph does not have $k$-bands of hexagons. It is maximal, i.e. $c_{Y_{\mathscr{G}}}=1$, due to our choice of the gluing. It is also projective by Proposition 2.28. Hence there is a deformation with the claimed properties by Proposition 2.13. Letting $f: \mathcal{Y}_{\mathscr{G}} \rightarrow S$ denote the structure morphism, we prove the vanishing of $H^{1}\left(\mathcal{Y}_{\mathscr{G}}, \mathcal{O}_{\mathcal{Y}_{\mathscr{G}}}\right)=0$ as follows: We have $H^{1}\left(Y_{\mathscr{G}}, \mathcal{O}_{Y_{\mathscr{G}}}\right)=0$ by [Fri83, Lemma 5.7] so by semi-continuity it follows from a result of Grauert [Har77, Cor 12.9] that $R^{1} f_{*} \mathcal{O}_{\mathcal{Y}_{\mathscr{G}}}=0$. As we work over an affine base, $H^{1}\left(\mathcal{Y}_{\mathscr{G}}, \mathcal{O}_{\mathcal{Y}_{\mathscr{G}}}\right)=0$ follows from [Har77, Proposition 8.5].

We give examples for low degree. 

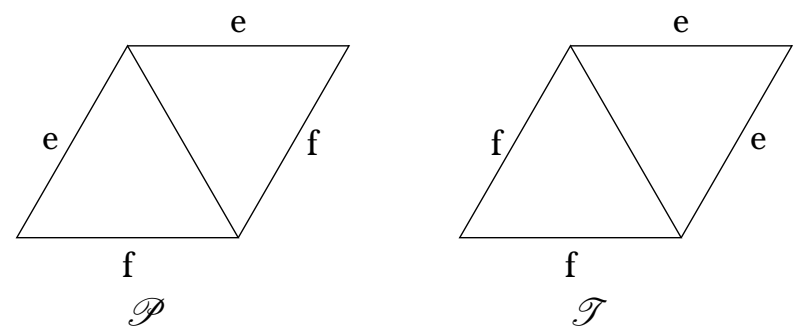

Figure 8. The triangulations $\mathscr{P}$ and $\mathscr{T}$.
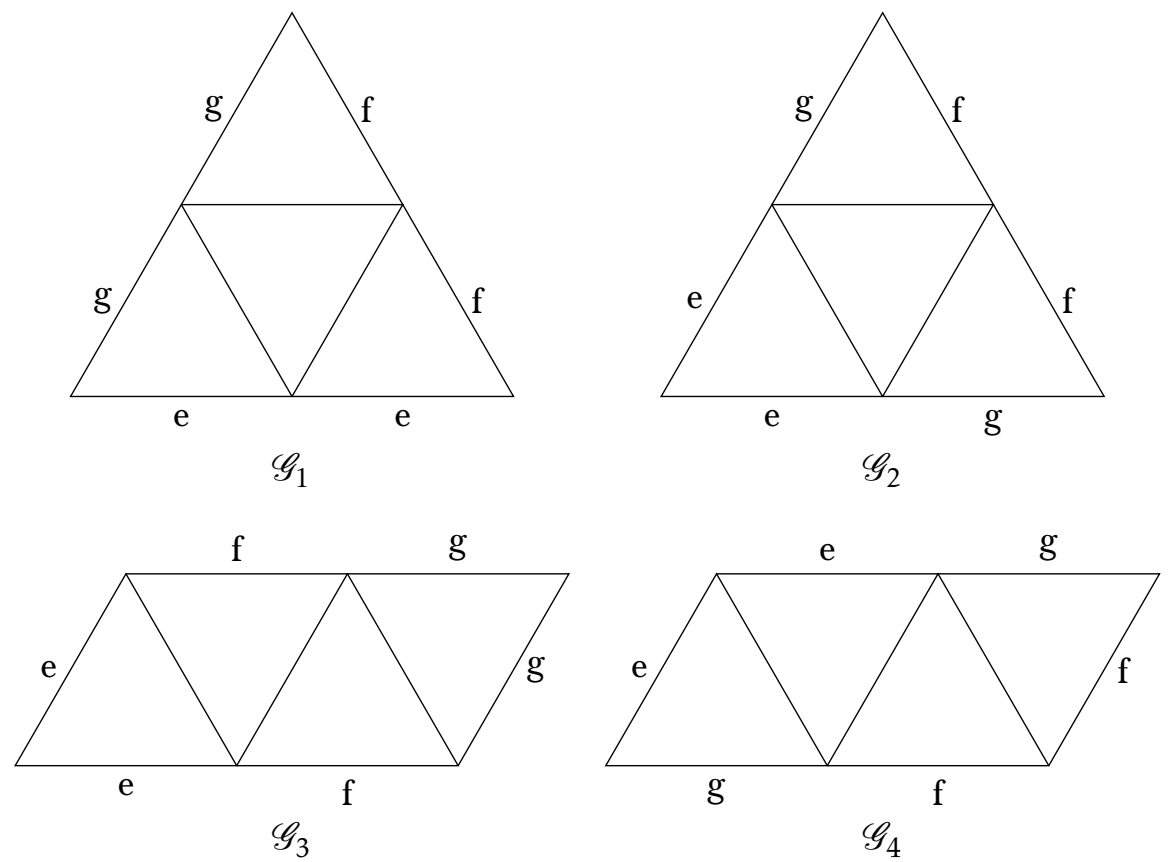

Figure 9. The triangulations with 4 triangles.

Example 2.30 ( $c f$. [Lie17]). Specialising to genus 2, there are precisely two different triangulations of $S^{2}$ with two triangles, corresponding to dual intersection complexes of degenerations with central fibres having three components. By e.g. [ES17], these are given by two triangles glued along the boundary and two triangles glued along one side to each other, with the remaining sides identified. We shall denote the first of these triangulations by $\mathscr{P}$ and the latter by $\mathscr{T}$, see Figure 8 . Let $Y_{\mathscr{P}}$ be the surface obtained by gluing three copies of $\biguplus_{2}$ by identifying components of the boundary cycle such that interior special points are identified. For the triangulation $\mathscr{T}$, the associated maximal surface is a copy of $\bigsqcup_{4}$, with two opposite components, say $D_{1}$ and $D_{3}$, of the anticanonical curve $D$ identified, and two copies of $\varphi_{1}$ glued to the (images) of $D_{2}$ and $D_{4}$, such that the images of the special points match.

Example 2.31. There are 4 combinatorially distinct triangulations of $S^{2}$ with 4 triangles, see [ES17, Example 2.5] and Figure 9. We collect the valencies of the vertices in a tuple. The tuples are $(3,3,3,3),(3,2,1,6),(5,5,1,1)$ and $(4,4,2,2)$. None of these triangulations have $k$-bands of hexagons for $k>1$, because the least number of triangles in such a triangulation is 8 . To take an example let $\mathscr{G}_{1}$ denote the triangulation such that every vertex has valency 3 . The resulting surface $Y_{\mathscr{G}_{1}}$ are three copies of $\bigcup_{3}$ glued according to the triangulation. Here, this means that each component meets each of the remaining components in a curve $C \cong \mathbb{P}^{1}$ in such a way that the special points are identified. 


\section{Relative Notions and the Mori fan}

Here we introduce the Mori fan which is the central object of our paper. We will now work in a relative setting over a fixed base.

\subsection{Relative Notions}

As we will make extensive use of various concepts of the minimal model program we will recall the relevant basic concepts here, following [Kaw97]. Let $\pi: \mathcal{X} \rightarrow U$ be a projective morphism of normal schemes. An $\mathbb{R}$-Cartier divisor $L$ on $\mathcal{X}$ is an $\mathbb{R}$-linear combination of Cartier divisors. A $\mathbb{Q}$-Cartier divisor $L$ on $\mathcal{X}$ is a $\mathbb{Q}$-linear combination of Cartier divisors. Similarly, an $\mathbb{R}$-Weil divisor is an $\mathbb{R}$-linear combination of prime divisors. Two $\mathbb{R}$-Cartier divisors $L, L^{\prime}$ are linearly equivalent over $U$ if their difference is an $\mathbb{R}$-linear combination of principal divisors and an $\mathbb{R}$-Cartier divisor pulled back from $U$. Two $\mathbb{R}$-Cartier diviors $L, L^{\prime}$ are numerically equivalent over $U$, denoted by $L \equiv_{U} L^{\prime}$, if $L \cdot C=L^{\prime} \cdot C$ for all curves - integral 1-dimensional closed subschemes $-C$ in fibres of $\pi$.

We define

$$
\operatorname{Pic}(\mathcal{X} / U)_{\mathbb{R}}=\{\mathbb{R} \text {-Cartier divisors on } X\} / \text { linear equivalence over } U
$$

and

$$
\mathrm{N}^{1}(\mathcal{X} / U)=\{\mathbb{R} \text {-Cartier divisors on } X\} / \text { numerical equivalence over } U \text {. }
$$

The latter is a finite dimensional vector space [Kle66, Proposition IV.4.3].

In this context, we call an element of $\mathrm{N}^{1}(\mathcal{X} / U)$ a $\mathbb{Z}$-divisor if we want to emphasise that it is the class of a Cartier divisor.

Remark 3.1. For a type III degeneration $\mathcal{Y} \rightarrow S$ of $\mathrm{K} 3$ surfaces, we have $\operatorname{Pic}(\mathcal{Y} / S)_{\mathbb{R}} \cong \mathrm{N}^{1}(\mathcal{Y} / S)$ : indeed, by maximality, it is enough to show this on the central fibre $Y$, where it follows from the fact that any divisor on the central fibre is given by an element of the lattice $L$ defined in Sequence 2.3 and all (normalised) components are smooth rational surfaces.

We say that a Cartier divisor $L$ is nef over $U$, or $\pi-n e f$, if $L \cdot C \geq 0$ for all curves $C$ in $\mathcal{X}$ mapping to a point. A Cartier divisor $L$ is ample over $U$, or $\pi$-ample, if the restriction $L_{u}$ is ample on the fibre $\mathcal{X}_{u}$ for all $u \in U$. A Cartier divisor $L$ is semi-ample over $U$, or semi-ample, if there is a projective morphism $g: \mathcal{Z} \rightarrow U$, a $U$-morphism $\mathcal{X} \rightarrow \mathcal{Z}$ and a $g$-ample divisor $A$ on $\mathcal{Z}$ such that $L^{\otimes n}=g^{*} A$ for some integer $n>0$. A Cartier divisor $L$ is $\pi$-movable (or $\pi$-moving) if $\operatorname{dim} \operatorname{Supp} \operatorname{Coker}\left(\pi^{*} \pi_{*} \mathcal{O}_{\mathcal{X}}(L) \rightarrow \mathcal{O}_{\mathcal{X}}(L)\right) \geq 2$.

3.1.1. Cones.- The $\pi$-nef cone $\operatorname{Nef}(\mathcal{X} / U)$ is the closure of the $\pi$-ample cone in $\mathrm{N}^{1}(\mathcal{X} / U)$, the closed $\pi$-movable cone $\overline{\mathcal{M}}(\mathcal{X} / U)$ is the closure of the convex cone generated by $\pi$-movable divisors. A Cartier divisor $L$ is $\pi$-effective if $\pi_{*} \mathcal{O}(L) \neq 0$. Let $\mathcal{B}^{e}(\mathcal{X} / U)$ be the convex cone generated by all $\pi$-effective divisors, and let $\overline{\mathcal{B}^{e}}(\mathcal{X} / U)$ denote its closure, the cone of pseudo-effective divisors. Set

$$
\operatorname{Mov}(\mathcal{X} / U)=\overline{\mathcal{M}}(\mathcal{X} / U) \cap \mathcal{B}^{e}(\mathcal{X} / U) .
$$

We will refer to $\operatorname{Mov}(\mathcal{X} / U)$ as the moving cone.

\subsection{The Mori fan}

The Mori fan was first introduced in [HK00]. Here we shall recall the definition in the situation which we consider, namely for models of the DNV family.

First however, we recall the pullback under dominant rational maps in general. For this we fix a dominant rational map $f: \mathcal{X} \rightarrow \mathcal{Z}$ of projective $\mathbb{Q}$-factorial schemes over $U$. Let $L$ be an effective Cartier divisor on $\mathcal{Z}$. We define $f^{*}(L)$ to be the unique Weil divisor that is equal to the pullback of $L$ by $f$ on the open set of 
codimension 2 where $f$ is a morphism. The divisor $f^{*}(L)$ is $\mathbb{Q}$-Cartier by $\mathbb{Q}$-factorality of $\mathcal{X}$. In this way we obtain a linear map $f^{*}: \mathrm{N}^{1}(\mathcal{Z} / U) \rightarrow \mathrm{N}^{1}(\mathcal{X} / U)$.

Now we specialize to the case where $\mathcal{Y} \rightarrow S$ is a model of the DNV family, of degree $2 d$, see also [GHKS]. Let $f: \mathcal{Y} \rightarrow \mathcal{Y}^{\prime}$ be a small modification over $S$, i.e. a birational map which is an isomorphism in codimension 1 such that $\mathcal{Y}^{\prime}$ is projective over $S$. In this situation, we will also write $\left(\mathcal{Y}^{\prime}, f\right)$ for $f: \mathcal{Y} \rightarrow \mathcal{Y}^{\prime}$ and call it a marked minimal model of the DNV family (where we have fixed $\mathcal{Y} \rightarrow S$ as a reference model).

Define the cone

$$
C(f):=f^{*} \operatorname{Nef}^{\mathrm{e}}\left(\mathcal{Y}^{\prime} / S\right) \subset \mathrm{N}^{1}(\mathcal{Y} / S)
$$

where

$$
\operatorname{Nef}^{\mathrm{e}}\left(\mathcal{Y}^{\prime} / S\right)=\operatorname{Nef}\left(\mathcal{Y}^{\prime} / S\right) \cap \operatorname{Eff}\left(\mathcal{Y}^{\prime} / S\right)
$$

is the effective nef cone.

Definition 3.2. Let $\mathcal{Y} \rightarrow S$ be a model of the DNV family of degree $2 d$. The set of all cones $C(f)$ and their faces, with $f$ a small modification, is the Mori fan of $\mathcal{Y} \rightarrow S$, denoted by $\operatorname{MF}(\mathcal{Y} / S)$.

Remark 3.3. If $\left(\mathcal{Y}^{\prime}, f\right)$ and $\left(\mathcal{Y}^{\prime \prime}, g\right)$ are two marked minimal models and $C(f) \cap C(g)$ has codimension 0 , then by [Kaw97, Lemma 1.5] there is an isomorphism $\beta: \mathcal{Y}^{\prime \prime} \rightarrow \mathcal{Y}^{\prime}$ with $f=\beta \circ g$, and hence $C(f)=C(g)$.

Note that if $\mathcal{Y}^{\prime} \rightarrow S$ is another model, $\operatorname{MF}\left(\mathcal{Y}^{\prime} / S\right)$ is canonically identified with $\operatorname{MF}(\mathcal{Y} / S)$ via the isomorphism $\mathrm{N}^{1}(\mathcal{Y} / S) \cong \mathrm{N}^{1}\left(\mathcal{Y}^{\prime} / S\right)$ induced by taking strict transforms. In particular, the Mori fan only depends on the DNV family, not on a specific model.

We recall the following result of [GHKS], which implies that $\mathrm{MF}(\mathcal{Y} / S)$ is indeed a fan, and, in particular, is closed under intersection and taking faces. If $\Sigma$ is a fan in a vector space $V$, then we denote its support by $|\Sigma|$.

Theorem 3.4 ( $c f$. [GHKS, Theorem 6.5]). The following holds:

(i) Let $\triangle \subset \operatorname{Nef}\left(\mathcal{Y}_{\eta}\right)$ be a rational polyhedral cone. Let $r_{\eta}$ be the restriction map $\operatorname{Pic}(\mathcal{Y} / S) \rightarrow \operatorname{Pic}\left(\mathcal{Y}_{\eta}\right)$. Then $r_{\eta}^{-1}(\triangle)$ and $r_{\eta}^{-1}(\triangle) \cap \operatorname{Mov}(\mathcal{Y} / S)$ are rational polyhedral cones and

$$
\left\{r_{\eta}^{-1}(\triangle) \cap \gamma \mid \gamma \in \operatorname{MF}(\mathcal{Y} / S)\right\}
$$

is a finite set of rational polyhedral cones, with support $r_{\eta}^{-1}(\triangle) \cap \operatorname{MF}(\mathcal{Y} / S)$.

(ii) The support of $\operatorname{MF}(\mathcal{Y} / S)$ is $\operatorname{Mov}(\mathcal{Y} / S)$.

Proof. We sketch the approach of [GHS16]. The crucial point is that one can construct models of the DNV family not only over $S$, but over the spectrum of the local ring of a smooth algebraic curve, namely the local ring of a cusp of the compactified Dolgachev mirror space which, in this case, is a modular curve. For these models one can run MMP, see [KM98, §3.6]. Then, invoking [Sho96], one concludes by [Kaw11, Theorems 3 and 4$]$.

Remark 3.5. The fact that one can run MMP for $\mathcal{Y} \rightarrow S$ implies that a small modification $f: \mathcal{Y} \rightarrow \mathcal{Y}^{\prime}$ factors into flops. Hence $\mathcal{Y}^{\prime} \rightarrow S$ is also a model of the DNV family, which justifies the use of the name marked model.

Remark 3.6. The proof we have just sketched also shows that log-abundance holds for models $\mathcal{Y} \rightarrow S$ of the DNV family. In particular nef line bundles are semi-ample.

Remark 3.7. The nef cone of $\mathcal{Y}_{\eta}$ is finitely polyhedral if and only if $d=1$. To see this we argue by means of the analytic category. Using the sequence (2.6) and its analytic analogue we can identify the nef cone of $\mathcal{Y}_{\eta}$ with the nef cone of a very general fibre of an analytic Kulikov model $\mathcal{X} \rightarrow \mathbb{D}$. The claim then follows from Nikulin's classification in [Nik81], as the Picard lattice of $\mathcal{Y}_{\eta}$ is $U \oplus 2 E_{8}(-1) \oplus\langle-2 d\rangle$. See [Nik14, Theorem 1] for a more explicit statement of Nikulin's classification. Hence the Mori fan of a model $\mathcal{Y} \rightarrow S$ of degree 2 is a finite collection of rational polyhedral cones. Note that this is special to the case $d=1$. 
3.2.1. Interior facets. - It is well known that the codimension 1 faces separating maximal (dimensional) cones of the Mori fan correspond to flopping contractions. We recall the details here. Let $\mathcal{Y}, \mathcal{Y}^{\prime}$ be models of the DNV family of degree $2 d$. Let $\phi: \mathcal{Y}-\rightarrow \mathcal{Y}^{\prime}$ be a flop. Then there exists a divisor $F$ on $\mathcal{Y}$ and morphisms $\psi, \psi^{\prime}$ with $\psi$ a contraction of an $F$-negative extremal ray such that the diagram

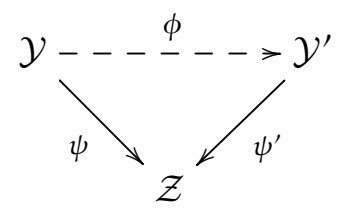

commutes. Write $\phi_{*} F=F^{\prime}$ for the birational transform. By the contraction theorem, we have

$$
\mathrm{N}^{1}(\mathcal{Y} / S)=\psi^{*} \mathrm{~N}^{1}(\mathcal{Z} / S) \oplus \mathbb{R}[F]
$$

and

$$
\mathrm{N}^{1}\left(\mathcal{Y}^{\prime} / S\right)=\left(\psi^{\prime}\right)^{*} \mathrm{~N}^{1}(\mathcal{Z} / S) \oplus \mathbb{R}\left[F^{\prime}\right]
$$

Using the isomorphism $\mathrm{N}^{1}(\mathcal{Y} / S) \cong \mathrm{N}^{1}\left(\mathcal{Y}^{\prime} / S\right)$ given by taking strict transforms under $\phi$, we can identify $\psi^{*} \mathrm{~N}^{1}(\mathcal{Z} / S)=\left(\psi^{\prime}\right)^{*} \mathrm{~N}^{1}(\mathcal{Z} / S)$. This cone spans a hyperplane in $\mathrm{N}^{1}(\mathcal{Y} / S)$ and $\mathbb{R}[F]=\mathbb{R}\left[F^{\prime}\right]$. Hence the cones $\operatorname{Nef}(\mathcal{Y} / S)$, which we can think of as $C\left(\operatorname{id}_{\mathcal{Y} / S}\right)$, and $C(\phi)$ in the Mori fan meet in codimension 1 , compare [Mat02, Proposition 12.2.2].

Conversely, we now assume that $\sigma, \sigma^{\prime}$ are two maximal cones of $\operatorname{MF}(\mathcal{Y} / S)$ with $\sigma \cap \sigma^{\prime}=\tau$ a codimension 1 face. Without loss of generality we can assume $\sigma=\operatorname{Nef}(\mathcal{Y})$. By the definition of the Mori fan there is a small modification $f: \mathcal{Y} \rightarrow \mathcal{Y}^{\prime}$ of projective minimal models with $f^{*}\left(\operatorname{Nef}\left(\mathcal{Y}^{\prime} / S\right)\right)=\sigma^{\prime}$. Let $\psi: \mathcal{Y} \rightarrow \mathcal{Z}$ be the contraction defined by the linear system of a suitable multiple of a very general effective divisor $L$ in $\tau$. Note that this is indeed a morphism as $L$ is semi-ample by abundance, see Remark 3.6. Then $\tau=\psi^{*}(\operatorname{Nef}(\mathcal{Z}))$. The facet $\tau$ defines an extremal ray $R$ in $\mathrm{N}^{1}(\mathcal{Y} / S)$ that is cut out by $L$, i.e. $\psi=\operatorname{contr}_{R}$. Also, any divisor in the interior of $\sigma^{\prime}$ is negative on $R$. Let $F \in \operatorname{MF}(\mathcal{Y} / S)$ be such a divisor. Then $\psi$ is an $F$-flopping contraction. Thus the diagram

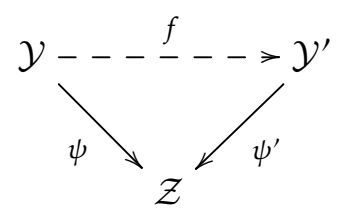

exhibits $f$ as an $F$-flop.

Definition 3.8. We say that a codimension 1 cone $\tau$ of $\operatorname{MF}(\mathcal{Y} / S)$ is an interior facet if there are two marked models $\left(\mathcal{Y}^{\prime}, f\right),\left(\mathcal{Y}^{\prime \prime}, g\right)$ of the DNV family such that $C(f) \cap C(g)=\tau$.

The above discussion can now be summarized as follows:

Proposition 3.9. Every facet of $\operatorname{Nef}(\mathcal{Y} / S) \in \operatorname{MF}(\mathcal{Y} / S)$ that is an interior facet of $\operatorname{MF}(\mathcal{Y} / S)$ defines a flop $\mathcal{Y} \rightarrow \mathcal{Y}^{\prime}$ and conversely any such flop is defined by an interior facet.

3.2.2. Action of $\operatorname{Bir}(\mathcal{Y} / S)$.- The birational $S$-automorphisms $\operatorname{Bir}(\mathcal{Y} / S)$ do not contract divisors, as $\mathcal{Y} \rightarrow S$ is a minimal model. Hence we can define a representation

$$
\sigma: \operatorname{Bir}(\mathcal{Y} / S) \rightarrow \operatorname{GL}\left(\mathrm{N}^{1}(\mathcal{Y} / S)\right)
$$

by $\sigma(\theta)(D)=\theta_{*}(D)$ for $\theta \in \operatorname{Bir}(\mathcal{Y} / S)$. Since $\theta$ is a small modification, this defines a permutation action on the set of maximal cones of $\operatorname{MF}(\mathcal{Y} / S)$. The following result, which will be crucial for us, follows directly as in [Kaw97, Lemma 1.5]. 
Proposition 3.10. Let $f_{i}: \mathcal{Y} \rightarrow \mathcal{Y}_{i}, i=1,2$ be small modifications of $\mathcal{Y}$ over $S$, with $\mathcal{Y}_{1}$ and $\mathcal{Y}_{2}$ models of the DNV family (of a given degree). Suppose that the associated Mori cones are in the same orbit under the action of $\operatorname{Bir}(\mathcal{Y} / S)$, i.e. that there is a birational automorphism $\theta \in \operatorname{Bir}(\mathcal{Y} / S)$ such that

$$
C\left(f_{2}\right)=\theta^{*} C\left(f_{1}\right) \text {. }
$$

Then there is an isomorphism $\beta: \mathcal{Y}_{1} \rightarrow \mathcal{Y}_{2}$ such that $\beta \circ f_{1}=f_{2}$.

\section{4. (-1)-Curves and Curve Structures}

In this section we will analyse the (-1)-curves in the components of a central fibre of a Kulikov model. This will lead to the definition of the concept of a curve structure for which we introduce several notions. Curve structures and their properties will be an essential tool in our analysis of the Mori fan.

From this section on, $Y_{c}$ will denote a $d$-semistable $K 3$ surface of type III while $Y$ will denote a component of $Y_{c}$. For any degree $2 d$, let $\mathrm{DNV}_{2 d}$ be the set of $d$-semistable $K 3$ surfaces of type III in $(-1)$-form with $t=2 d$ triple points. For $d=1$, these are the surfaces $Y_{\mathscr{P}}$ and $Y_{\mathscr{T}}$ described in Example 2.30. Let $\operatorname{Mod}_{2 d}$ denote the set of surfaces $Y_{c}$ such that there is $Y_{0} \in \mathrm{DNV}_{2 d}$ and a sequence of elementary modifications of type I

$$
Y_{0} \rightarrow Y_{1} \rightarrow \cdots \rightarrow Y_{i} \rightarrow \cdots \rightarrow Y_{n}=Y_{c} .
$$

This also defines an anticanonical divisor on each component of $Y_{c}$. We denote by $\operatorname{PMod}_{2 d}$ the subset of projective surfaces in $\operatorname{Mod}_{2 d}$. Each of these surfaces determines a model of the Dolgachev family of degree $2 d$. In general, one does not obtain the full set of models in this way as, usually, a central fibre of a model of the DNV family of degree $2 d$ cannot be obtained by type I modifications alone. However, we will later see that for $d=1$ the set $\mathrm{PMod}_{2}$ parametrizes the isomorphism classes of models of the Dolgachev-Nikulin-Voisin family of degree 2, see Corollary 6.33. We shall also see in Section 7 that $\operatorname{Mod}_{2}$ is finite, but we do not expect $\operatorname{Mod}_{2 d}$ to be finite for higher degree, see also Remark 3.7.

Definition 4.1. Let $Y_{c} \in \operatorname{Mod}_{2}$. We say $Y_{c}$ is of class $\mathscr{T}$ (resp. $\mathscr{P}$ ) if the dual intersection graph of $Y_{c}$ is given by $\mathscr{T}$ (resp. $\mathscr{P})$.

We denote the set of $Y_{c} \in \operatorname{Mod}_{2}$ of class $\mathscr{T}($ resp. $\mathscr{P})$ by $\operatorname{Mod}_{2}(\mathscr{T})\left(\operatorname{resp} \cdot \operatorname{Mod}_{2}(\mathscr{P})\right)$, and similarly we define $\operatorname{PMod}_{2}(\mathscr{T})$ and $\operatorname{PMod}_{2}(\mathscr{P})$. Note that this defines decompositions into disjoint unions

$$
\operatorname{Mod}_{2}=\operatorname{Mod}_{2}(\mathscr{T}) \sqcup \operatorname{Mod}_{2}(\mathscr{P}) \text { and } \operatorname{PMod}_{2}=\operatorname{PMod}_{2}(\mathscr{T}) \sqcup \operatorname{PMod}_{2}(\mathscr{P}) \text { respectively. }
$$

We recall the following elementary facts about anticanonical pairs, i.e. pairs $(Y, D)$ with $Y$ a smooth rational surface and $D$ an effective anticanonical cycle. We will use these without further mention.

Proposition 4.2. Let $(Y, D)$ be an anticanonical pair. Let $C$ be an irreducible curve that is not a component of D.

(i) If $C^{2}=-1$, then $C \cdot D=1$ and $C$ is smooth rational.

(ii) If $C^{2}=0$, then $C \cdot D=2$ or $C \cdot D=0$. In the first case, $C$ is smooth rational, in the second case $p_{a}(C)=1$.

(iii) If $C^{2}=-2$, then $C$ is smooth rational and $C \cdot D=0$.

\section{1. (-1)-Curves}

In order to control flops, we need to control exceptional curves of the first kind on the components of the central fibres. We show that the set of possible elementary modifications of a surface in $\operatorname{Mod}_{2}$ is quite small. Recall the following terminology. A cycle is a graph whose vertices and edges can be ordered $C_{1}, \ldots, C_{n}$ and $e_{1}, \ldots, e_{n}$ such that $e_{i}$ connects $C_{i}$ and $C_{i+1}$ (where the indices have to be read cyclically). A tree is a connected graph not containing a subgraph that is a cycle. A vertex $v$ of a graph is called a fork if there are 


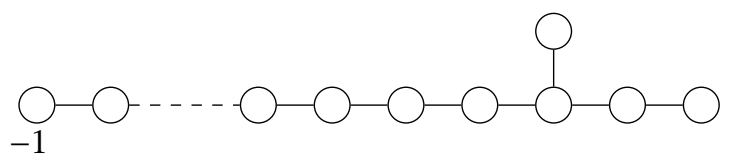

Figure 10. The intersection graph $\Gamma_{1}^{\mathrm{n}}$. All vertices with label not displayed are labelled with -2 .

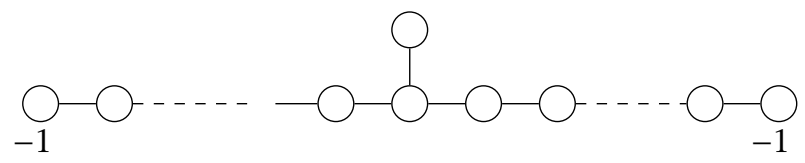

Figure 11. The intersection graph $\Gamma_{2}^{\mathbf{n}}$. All vertices with label not displayed are labelled with -2 .

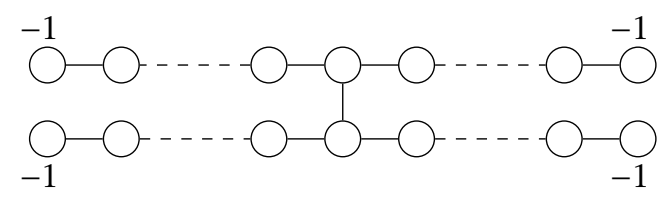

Figure 12. The intersection graph $\Gamma_{4}^{\mathrm{n}}$. All vertices with label not displayed are labelled with -2 .

at least three edges from $v$. If $G$ is a tree with a unique fork $v \in G$, the connected components of $G \backslash\{v\}$ are the branches of $v$.

Construction 4.3. Let $\Gamma_{1}^{0}$ be the graph underlying the extended Dynkin diagram $\tilde{E}_{8}$, i.e. a tree with a unique fork $v$ and 3 branches $B_{i}, i=1,2,3$, consisting of 1,2 and 5 vertices. Let $\mathbf{n}=n_{1} \geq 0$. Let $\Gamma_{1}^{\mathbf{n}}$ be the tree where we replace the leg $B_{3}$ with 5 vertices by a leg with $5+n_{1}$ vertices. We label the end of this branch with -1 , all other vertices are labelled with -2 , see Figure 10. (We shall later interpret these labels as intersection numbers.)

Let $\Gamma_{2}^{0}$ be the graph underlying the extended Dynkin diagram $\tilde{E}_{7}$ diagram, i.e. the graph with a unique fork $f$ and 3 branches $B_{i}, i=1,2,3$, consisting of 1,3 and 3 vertices. Let $\mathbf{n}=\left(n_{1}, n_{2}\right)$. We define $\Gamma_{2}^{\mathbf{n}}$ to be the tree where we replace the two branches $B_{2}, B_{3}$ of length 3 by branches of length $3+n_{1}$ and $3+n_{2}$ respectively. We label the vertices at the end of these branches with -1 and all other vertices with -2 , see Figure 11.

Finally, let $\Gamma_{4}^{0}$ be the graph underlying the extended Dynkin diagram $\tilde{D}_{5}$, i.e. the unique tree with 2 forks and 6 vertices. Label the vertices that are not forks from 1 to 4 such that one fork is connected to the vertices labelled 1 and 2 . Let $\mathbf{n}=\left(n_{1}, n_{2}, n_{3}, n_{4}\right)$ and define $\Gamma_{4}^{\mathbf{n}}$ to be the graph where we replace the vertex labelled $i \in\{1,2,3,4\}$ by a leg of length $n_{i}+1$. Again, we label the ends of the branches with -1 and all other vertices with -2 , see Figure 12 .

The following lemma is a helpful technical tool, which we will employ repeatedly. Note that we make no assumption on $C_{n}^{2}$ in this lemma.

Lemma 4.4. Let $n \in \mathbb{N}, n \geq 2$. Let $C_{i}, i=1, \ldots, n$ be a collection of curves on a smooth surface $Y$. Suppose the dual graph is $A_{n}$, with labelling of the vertices $v_{i}$ as in Figure 13. Assume the labelling is such that $v_{i}$ corresponds to the curve $C_{i}$. Moreover, assume $C_{i}^{2}=-1$ if $i=1$ and -2 if $1<i<n$. Let $\mathcal{H}$ be a set of curves on $Y$ such for all $h \in \mathcal{H}, h \cdot C_{i}=0$ for $i=1, \ldots, n-1$. Set $A=\sum_{i=1}^{n} a_{i} C_{i}+\sum_{h \in \mathcal{H}} \gamma_{h} h$ for $a_{i}, \gamma_{h} \in \mathbb{Q}$ and assume $A \cdot C_{i} \geq 0$ for $i=1, \ldots, n-1$ and $a_{1} \geq 0$. Then $a_{i+1} \geq a_{i}$. If $A \cdot C_{i}>0$ for $i=1, \ldots, n-1$ and $a_{1} \geq 0$, it followes $a_{i+1}>a_{i}$.

Proof. We have $0 \leq A \cdot C_{1}=-a_{1}+a_{2}$, so $a_{2} \geq a_{1}$. Suppose $a_{i} \geq a_{i-1}$ for some $1<i<n-1$. Then $0 \leq A \cdot C_{i}=a_{i+1}-2 a_{i}+a_{i-1}$, so $a_{i+1} \geq 2 a_{i}-a_{i-1} \geq a_{i}$. The claim follows. Replacing weak by strict inequalites shows the second claim. 


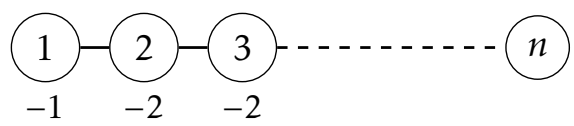

Figure 13. The numbering of the vertices of the $A_{n}$ graph in Lemma 4.4.

Let $\bigcup_{i}$ be as in Construction 2.25, with anticanonical cycle $D$, and let $p$ be a special point. We recall that $p$ is an interior special point if $p$ is a smooth point of $D$.

Proposition 4.5. Consider $\bigcup_{i}$ for $i=1,2$ or 4 , with anticanonical divisor D. Let $\left(p_{1}, \ldots, p_{i}\right)$ denote the tuple of interior special points. Let $Y$ denote the $\mathbf{n}=\left(n_{1}, \ldots, n_{i}\right)$ blow-up of $\bigsqcup_{i}$ in $\left(p_{1}, \ldots, p_{i}\right)$.

Then there are exactly $i$ exceptional curves of the first kind $C_{k} \subset Y($ for $k=1, \ldots, i)$ such that $C_{k}$ is not a component of D. More precisely, the intersection graph of the negative curves that are not components of $D$ is given by the graph $\Gamma_{i}^{\mathrm{n}}$.

Proof. Let $\psi: Y \rightarrow \bigcup_{i}$ be the $\left(n_{1}, \ldots, n_{i}\right)$ blow-up of $\bigcup_{i}$ in the interior special points $\left(p_{1}, \ldots, p_{i}\right)$.

Let $C(Y)$ be the set consisting of strict transforms of the $(-1)$ curves $E_{i}$ from the construction of the $\bigcup_{i}$ in Construction 2.25 and the (-2)-curves of $y_{i}$ together with the irreducible components of the exceptional locus of $\psi$, see also Figures 6 and 7. By the results of [Loo81, Section 2], the set $C(Y)$ defines a $\mathbb{Q}$-basis of $\operatorname{Pic}(Y)$, with intersection graph $\Gamma_{i}^{\mathbf{n}}$ and $\mathbf{n}=\left(n_{1}, \ldots, n_{i}\right)$. We can assume $i=4$ as it is straightforward to check, using the construction of the $\bigcup_{i}$, that the result for $i=4$ implies the result for $i=1,2$.

Let $C_{k}, k=1, \ldots, 4$ denote the integral $(-1)$-curves in $\operatorname{Ex}(\psi)$, corresponding to the vertices labelled with $(-1)$ in the diagrams. Let $D_{1}, \ldots, D_{4}$ denote the components of the anticanonical divisor $D$ given as birational transforms of the anticanonical cycle from the construction of $\bigsqcup_{4}$. Suppose there is an integral curve $C$ with $C^{2}<0$ not in $\mathcal{B}:=\left\{D_{1}, \ldots, D_{4}\right\} \cup C(Y)$. We have $C \cdot B \geq 0$ for all curves $B$ in $\mathcal{B}$. Write

$$
m C=\sum_{B \in C(Y)} \beta_{B} B
$$

with $m, \beta_{B} \in \mathbb{Z}$ and $m>0$. We can use Lemma 4.4 to show that all coefficients $\beta_{B}$ are non-negative. To see that we are in a position to use Lemma 4.4 we intersect with the curves $D_{i}$. Indeed, given a curve $D_{i}$ there is a unique (-1)-curve $E$ among the $C_{k}$ with $E \cdot D_{i} \neq 0$, in fact $E \cdot D_{i}=1$. Also $D_{i} \cdot H=0$ for all (-2)-curves. Hence $\beta_{E}=\beta_{E} E \cdot D_{i}=\sum_{\beta} \beta_{B} B \cdot D_{i}=m C \cdot D_{i} \geq 0$.

It now follows that

$$
0>m C^{2}=\sum_{B \in C(Y)} \beta_{B}(C \cdot B) \geq 0,
$$

where the last inquality follows since $\beta_{B} \geq 0$ and $C \cdot B \geq 0$. Hence a curve such as $C$ cannot exist.

Remark 4.6. The same proof works for $i=3$, the case $i=5$ also holds. We restrict to $i=1,2,4$ as these are the relevant surfaces in the $d=1$ case.

We have the following result.

Corollary 4.7. Let $Y_{c}=Y_{1} \cup Y_{2} \cup Y_{3}$ be in $\operatorname{Mod}_{2}$. Then the cones of curves $\operatorname{NE}\left(Y_{i}^{v}\right)$ on the normalisations of the components are finitely generated. If the Picard rank of $Y_{i}$ is at least 3 , a generating set is given by the curves $C$ with $C^{2}<0$. For smaller Picard rank, either $Y_{i}$ is a Hirzebruch surface or $Y_{i} \cong \mathbb{P}^{2}$.

Proof. The only thing that remains to show is the statement on the generators of cones of curves. By Proposition 4.5, there are finitely many curves $C$ on $Y_{i}$ with $C^{2}<0$, so, if the Picard rank is at least 3, the corollary follows from [AL11]. 


\subsection{Curve Structures}

Let $Y$ be a component of a surface $Y_{c} \in \operatorname{Mod}_{2}$. By definition, there is a sequence of type I flops $Y_{\mathscr{G}} \rightarrow Y_{c}$ whose inverse connects $Y$ to a component of a model $Y_{\mathscr{G}} \in \mathrm{DNV}_{2}$ in (-1)-form. Using this sequence, we will recursively define a set of curves $C(Y)$ on the normalisation $Y^{v}$. Note that there is an induced sequence of blow-ups and blow-downs $\psi: Y^{v} \rightarrow \bigsqcup_{i}$ for some $i \in\{1,2,4\}$.

Construction 4.8. Let $D$ denote the anticanonical divisor of $\biguplus_{i}$ as discussed in Construction 2.25. Set

$$
C\left(\mathfrak{y}_{i}\right):=\left\{C \subset \bigcup_{i} \mid C \text { is an integral curve with } C^{2}<0, C \not \subset D\right\} .
$$

To start the induction, we factor $\psi$ as $Y^{v} \rightarrow W \rightarrow \bigcup_{i}$ with $Y^{v} \rightarrow W^{v}$ corresponding to an elementary modification of type I. Suppose we have defined $C(W)$. If $Y^{v}=\mathrm{Bl}_{p}\left(W^{v}\right)$ for some $p$, we take $C(Y)$ to be the strict transforms of curves in $C(W)$ together with the exceptional curve of $Y^{v} \rightarrow W^{v}$. If $Y^{v} \rightarrow W^{v}$ is the inverse of $\pi: W^{v}=\operatorname{Bl}_{p}\left(Y^{v}\right) \rightarrow Y^{v}$, we set

$$
C(Y):=\left\{C \subset Y^{v} \mid C=\pi\left(C^{\prime}\right) \text { for } C^{\prime} \in C(W)\right\} .
$$

Because the normalisation morphism $Y^{v} \rightarrow Y$ identifies components of the singular locus of $Y_{c}$, which are part of the anticanonical cycle, and thus never curves in $C(Y)$, we can also interpret $C(Y)$ as a collection of curves on $Y$. We then proceed by induction.

The sequence $Y_{\mathscr{G}} \rightarrow Y_{C}$ is not uniquely defined, but the set $C(Y)$ and its intersection graph is independent of the choice of such a sequence, as the following lemma shows.

Lemma 4.9. Let $Y$ be a component of $Y_{c} \in \operatorname{Mod}_{2}$. The collection $C(Y)$ and the intersection graph are independent of the sequence $Y_{\mathscr{G}} \rightarrow Y_{c}$.

Proof. First, the surface $\bigcup_{i}$ is uniquely determined by the number of components of the double locus $D$ of $Y$. Let $D=D_{1}+\cdots+D_{i}$ and $p_{1}, \ldots, p_{i}$ be the interior special points. Let $\bar{D}=\bar{D}_{1}+\cdots+\bar{D}_{i}$ be the anticanonical cycle on $\bigsqcup_{i}$.

It follows by induction on the number of elementary modifications that we can write the induced sequence $Y^{v} \rightarrow \bigsqcup_{i}$ as

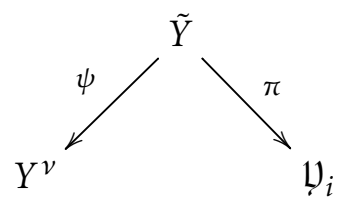

with $\pi$ the blow-up in the interior special points on components $\bar{D}_{i}$ of $\bar{D}$ such that their strict transform $D_{i}$ under $Y \rightarrow \bigcup_{i}$ has $D_{i}^{2}<-1$. Up to trivial modifications, i.e. blowing up a special point and then blowing down the resulting exceptional curve, this morphism is uniquely defined by the self-intersection numbers of $Y^{v}$. Let $J=\left\{j_{1}, \ldots, j_{k}\right\}$ be the set of indices of components $D_{j}$ of $D$ with $D_{j}^{2}>-1$, where we calculate intersection numbers on the normalisation. Then $\psi: \tilde{Y} \rightarrow Y^{v}$ is the $\left(n_{j_{1}}, \ldots, n_{j_{k}}\right)$-blow-up of $Y^{v}$ in $\left(p_{j_{1}}, \ldots, p_{j_{k}}\right)$. This uniquely defines the curves that are contracted.

Definition 4.10. Let $Y$ be as above. We define $\Gamma_{Y}$ to be the dual graph of $C(Y)$ and label its vertices with the self-intersection numbers. We call $\Gamma_{Y}$ the curve structure of $Y$. We say that a curve structure has type $d_{i}$ if $Y$ maps to $\bigcup_{i}$ under a sequence of type I flops. This is well defined as the number of components of $D$ is fixed under type I flops. Note that the chosen anticanonical divisor of $\varliminf_{i}$ has $i$ components.

Remark 4.11. We will usually consider $C(Y)$ as a set of curves on $Y^{v}$. In particular, intersection numbers will always be calculated on $Y^{v}$.

Remark 4.12. Note that for surfaces $Y$ which are $(n, m)$-blow-ups of some $\bigcup_{i}$ this coincides with the set $C(Y)$ which we have used in the proof of Proposition 4.5. 


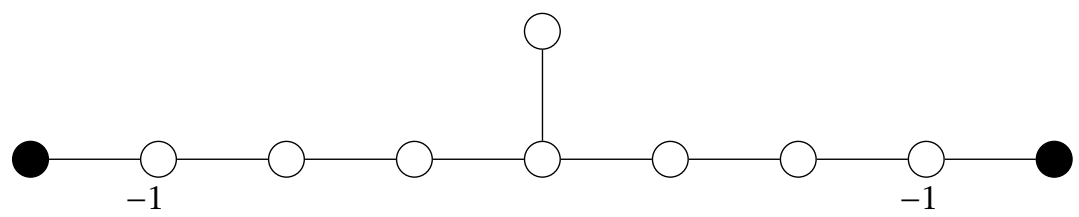

Figure 14. The augmented curve structure of a component $Y$ of $Y_{\mathscr{P}}$. The black vertices correspond to the components of the anticanonical divisor.

We will often interpret $\Gamma_{Y}$ as the underlying set of vertices. Also, for any vertex $v \in \Gamma_{Y}$, we will denote the underlying curve in $C(Y)$ by $C_{v}$, but abusing notation, we will often just write $v$. Note that if $v, w$ are two distinct vertices of $\Gamma_{Y}$, the number of edges between $v$ and $w$ is either 1 or 0 and this is the same as the intersection number $C_{w} \cdot C_{v}$. Two vertices $v, w$ with $v \cdot w=1$ for the underlying curves are called adjacent.

Given $Y$ with normalisation $Y^{v}$ and with curve structure $\Gamma_{Y}$, let $D=\sum D_{i}$ be the anticanonical divisor of $Y^{v}$. For each $D_{i}$, append a vertex $v_{D_{i}}$ to $\Gamma_{Y}$ and for each $v \in \Gamma_{Y}$ such that $C_{v}$ is not a component of $D$, add an edge joining $v_{D_{i}}$ and $v$ if $D_{i} \cdot C_{v} \neq 0$. This defines the augmented curve structure $\Gamma_{Y}^{a}$. See Figure 14 for an example.

Note that the existence of an edge between a vertex $v \in \Gamma_{Y}$ and a vertex corresponding to some $D_{i}$ does not necessarily imply that the intersection number of the corresponding curves is exactly 1 , it simply means that the intersection is non-empty.

If $\Gamma_{Y}$ and $\Gamma_{Y^{\prime}}$ are two curve structures, we then have an obvious notion of an isomorphism $\Gamma_{Y} \rightarrow \Gamma_{Y^{\prime}}$ : a bijection of graphs $\Gamma_{Y} \rightarrow \Gamma_{Y}$, preserving the intersection numbers that extends to a bijection of graphs $\Gamma_{Y}^{a} \rightarrow \Gamma_{Y^{\prime}}^{a}$.

Definition 4.13. Let $v_{e} \in \Gamma_{Y}$ be a vertex with $v_{e}^{2}=-1$. Let $D_{0}$ be the unique component of the anticanonical cycle met by $v_{e}$. The vertex $v_{e}$ is called exceptional if

$$
\left\{v \in \Gamma_{Y} \mid v \cdot D_{0} \neq 0\right\}=\left\{v_{e}\right\} \text { and }\left|\left\{v \in \Gamma_{Y} \mid v \cdot v_{e}=1\right\}\right|=1 .
$$

Let $Y$ be a component of a surface $Y_{c} \in \operatorname{Mod}_{2}$ with curve structure $\Gamma_{Y}$. We will also denote the preimage of the double locus of $Y_{c}$ on $Y$ under the normalisation map $Y^{v} \rightarrow Y$ by $D$. Let $v_{e} \in \Gamma_{Y}$ be an exceptional vertex. Starting with this exceptional vertex we now define a subgraph of $\Gamma_{Y}$ inductively. Since $v_{e}$ is exceptional, there is a unique vertex $v_{1}$ with $v_{1} \cdot v_{e}=1$. Set $L_{1}\left(v_{e}\right):=\left(v_{e}, v_{1}\right)$. Now suppose that we have already defined the ordered tuple of vertices $L_{n}\left(v_{e}\right)=\left(v_{e}, v_{1}, \ldots, v_{n}\right)$. If there is a unique vertex $v \in \Gamma_{Y} \backslash\left\{v_{e}, \ldots v_{n}\right\}$ adjacent to $v_{n}$ and $v_{n} \cdot D=0$, set $v_{n+1}:=v$ and $L_{n+1}\left(v_{e}\right)=L_{n}\left(v_{e}\right) \cup\left\{v_{n+1}\right\}$. Else set $L\left(v_{e}\right):=L_{n}\left(v_{e}\right)$. There is a unique connected subgraph of $\Gamma_{Y}$ whose vertices are given by the vertices in $L\left(v_{e}\right)$. By abuse of notation we consider $L\left(v_{e}\right)$ as this subgraph.

Definition 4.14. Let $v_{e}$ be an exceptional vertex. The graph $L\left(v_{e}\right)$ is the leg defined by $v_{e}$. The unique vertex $v$ of $L\left(v_{e}\right)$ not equal to $v_{e}$ meeting precisely one vertex of $L\left(v_{e}\right)$ is the end of the leg. In this situation we also say that $L\left(v_{e}\right)$ ends in $v$.

Definition 4.15. A curve structure $\Gamma_{Y}$ is called degenerate if there is no exceptional vertex, or if for some exceptional vertex $v_{e}$ the leg $L\left(v_{e}\right)$ ends in a vertex $v$ with $v \cdot D_{i} \geq 1$ for some component $D_{i} \subset D$ such that $D_{i}$ is in the preimage of a smooth component of the restriction of the double locus of $Y_{c}$ to $Y$. Curve structures that are not degenerate will be called non-degenerate.

The degenerate curve structures of type $d_{2}$ without exceptional vertices are displayed in Figure 15 .

Example 4.16. A non-degenerate curve structure $\Gamma_{Y}$ has at least one exceptional vertex. If $\Gamma_{Y}$ is of type $d_{2}$, then $Y$ is obtained from $\bigcup_{2}$ by a sequence of blow-ups and blow-downs. It follows from the definitions that $\Gamma_{Y}$ has two exceptional vertices. More precisely, $\Gamma_{Y}$ has two legs that both end on the same vertex $v$. 

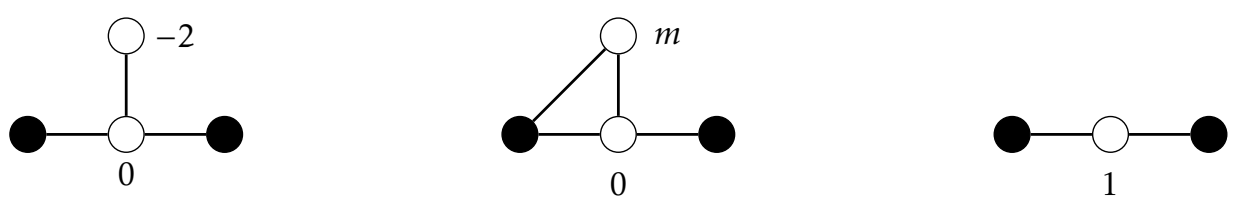

Figure 15. The possible (augmented) degenerate curve structures of type $d_{2}$ without exceptional vertex. The numbers give the self intersection of the underlying curve, with $m \geq-1$.

Moreover, there is a unique vertex $v^{\prime}$ that is on none of the legs and also meets $v$. In particular, we have $D_{1}^{2} \leq 1$ and $D_{2}^{2} \leq 1$ for the two components $D_{1}$ and $D_{2}$ of the anti-canonical divisor.

Proposition 4.17. Let $\Gamma_{Y}$ be a curve structure of type $d_{i}$ with $i \in\{1,2,4\}$. Then

$$
\left\{C_{v} \mid v \in \Gamma_{Y}\right\}
$$

is a $\mathbb{Q}$-basis of $\operatorname{Pic}\left(Y^{v}\right)$.

Proof. The statement is true for $\bigcup_{i}$ : as mentioned earlier, by the results in [Loo81, Section 2], one obtains a $\mathbb{Q}$-basis of $\operatorname{Pic}\left(\bigcup_{i}\right)$. In the general case, the curve structures in question are obtained via blow-ups and blow-downs from the $\bigcup_{i}, i \in\{1,2,4\}$. Arguing inductively, we need to show that given a curve structure $C\left(Y_{0}\right)$, the structure $C(Y)$ obtained on $Y$ under one of these operations is also a $\mathbb{Q}$-basis. Obviously, a blow-up $Y^{v} \rightarrow Y_{0}^{v}$ induces a curve structure on $Y^{v}$ that is also a $\mathbb{Q}$-basis, as it adds the exceptional curve. So let $g: Y_{0}^{v} \rightarrow Y^{v}$ be the blow-down in an exceptional curve corresponding to a type I flop, i.e. the contraction of an extremal ray spanned by a curve $E$. We have the exact sequence

$$
0 \rightarrow \operatorname{Pic}\left(Y^{v}\right) \rightarrow \operatorname{Pic}\left(Y_{0}^{v}\right) \rightarrow \mathbb{Z} \rightarrow 0
$$

with first map pullback via $g$ and second map evaluation on $E$. Let $A=\left\{H_{i}\right\}$ denote the collection of elements of $C\left(Y_{0}\right)$ disjoint from $E$. They induce a collection $\left\{L_{i}\right\}$ with $H_{i}=g^{*} L_{i}$. If $C_{j}$ is in $C\left(Y_{0}\right)$ but not in $A$ and not equal to $E$, then $C_{j} \cdot E=1$. Hence $C_{j}+E=g^{*}\left(F_{j}\right)$ for some $F_{j}$. The collection $\left\{L_{i}, F_{j}\right\}$ is linearly independent as $C\left(Y_{0}\right)=\left\{H_{i}, C_{i}+E, E\right\}$ is a spanning set of $\operatorname{Pic}\left(Y_{0}^{v}\right)_{\mathbb{Q}}$ with $\operatorname{rank} \operatorname{Pic}\left(Y_{0}^{v}\right)$ elements, and thus linearly independent. Also, note that $g_{*} H_{i}=g_{*} g^{*} L_{i}=L_{i}$ and $g_{*}\left(C_{j}\right)=g_{*}\left(C_{j}+E\right)=g_{*} g^{*}\left(F_{j}\right)=F_{j}$.

We now specialise the discussion to curve structures of type $d_{2}$. These are the ones appearing on components $Y$ of surfaces $Y_{c}$ in $\operatorname{Mod}_{2}(\mathscr{P})$. Note that in this case all components of $Y_{c}$ are smooth. Our goal in the remainder of this section is to express properties of the ample cone of such $Y$ in terms of curve structures. In the next section, we will see how these properties give criteria for the projectivity of $Y_{c}$. Note that all such $Y$ are smooth rational surfaces and the anticanonical divisor $D$ is the restriction of the double locus of $Y_{c}$ to $Y$.

Definition 4.18. Let $\Gamma_{Y}$ be a curve structure of type $d_{2}$. We say $\Gamma_{Y}$ is regular if $\left|\Gamma_{Y}\right|>1$ and no leg $L(e)$ of an exceptional vertex $e$ ends in a vertex $v$ with $v^{2}=0$. A curve structure which is not regular is called very degenerate and a curve structure which is regular and degenerate is called tamely degenerate.

In particular a non-degenerate curve structure is regular and a very degenerate curve structure is degenerate.

We have the following result.

Proposition 4.19. Let $Y$ be a component of $Y_{c} \in \operatorname{Mod}_{2}$. Suppose $\Gamma_{Y}$ is of type $d_{2}$ and write $D=D_{1}+D_{2}$.

(i) The intersection numbers $D_{1}^{2}$ and $D_{2}^{2}$, together with the type of the curve structure (regular or very degenerate), determine $Y$.

(ii) $\Gamma_{Y}$ is degenerate if and only if $D_{i}^{2} \geq 2$ for some $i \in\{1,2\}$.

(iii) If $\Gamma_{Y}$ is very degenerate, then after possibly relabelling the $D_{i}$ we have $D_{1}^{2}=4$ and $D_{2}^{2} \leq 1$. 


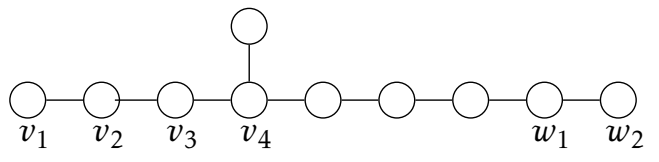

Figure 16. The intersection graph $\Gamma_{2}^{(0,2)}$. Self-intersection numbers are -1 for $v_{1}$ and $w_{2}$ and -2 for all remaining vertices. The $w_{i}$ are the exceptional locus of $\pi$ and the $v_{i}$ are the exceptional locus of $\psi$.

(iv) $\Gamma_{Y}$ is non-degenerate if and only if $D_{i}^{2} \leq 1$ for $i \in\{1,2\}$.

Proof. There is a sequence of elementary modifications $Y_{\mathscr{P}} \rightarrow Y$. Let $p_{1}, p_{2}$ be the interior special points. As in Lemma 4.9, there is a diagram

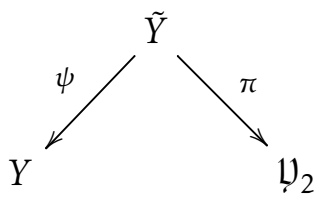

with $\pi$ the $(\mathrm{n}, \mathrm{m})$-blow-up in interior special points of $\bigcup_{2}$. Write $\bar{D}_{1}+\bar{D}_{2}$ for the anticanonical cycle on $\bigsqcup_{2}$ constructed in 2.25. Let $q_{1}, q_{2}$ be the interior special points on $\tilde{Y}$, i.e. the points where the birational transform of $D_{i}^{\prime}$ of $\bar{D}_{i}$ are met by an interior (-1)-curve. We can assume that there is no $q_{i}$ in $\operatorname{Ex}(\psi) \cap \operatorname{Ex}(\pi)$, as such points correspond to trivial modifications, i.e. blowing up and then blowing down the resulting curve. Let $I \subset\{1,2\}$ be the set of indices such that $q_{i}$ is not in $\operatorname{Ex}(\pi)$. Then $\psi$ is the blow down of disjoint trees of square negative curves $E_{1}+E_{2}+\cdots+E_{k}$ such that $E_{1}^{2}=-1, E_{i}^{2}=-2, i \geq 2$ and $E_{1} \cap D_{i}^{\prime}=\left\{q_{i}\right\}$ for an $i \in I$. This implies the proposition.

Remark 4.20. Note that (iii) in Proposition 4.19, if $D_{1}^{2}=4$ and $D_{2}^{2}=1$ then $Y \stackrel{\sim}{\rightarrow} \mathbb{P}^{2}$. The anti-canonical divisor then consists of a conic and a line.

For later use we record the following corollary.

Corollary 4.21. Let $Y_{c}=Y_{1} \cup Y_{2} \cup Y_{3} \in \operatorname{Mod}_{2}$ be such that $\Gamma_{Y_{i}}$ is of type $d_{2}$ and non-degenerate for $i=1,2$. Then $D_{12}^{2}, D_{21}^{2} \in[-3,1]$.

We illustrate Proposition 4.19 in the following example.

Example 4.22. Let $Y$ be a component of $Y_{c} \in \operatorname{Mod}_{2}$. Suppose $\Gamma_{Y}$ is of type $d_{2}$. Write $D=D_{1}+D_{2}$ and let $p_{1}, p_{2}$ be the corresponding interior special points. Suppose $D_{1}^{2}=3$ and $D_{2}^{2}=-3$. Write

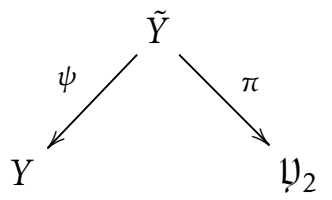

as above. Here, $\pi$ is the $(n, m)$-blow up of $\bigcup_{2}$ in $\left(\bar{p}_{1}, \bar{p}_{2}\right)$. Choose indices such that $\bar{D}_{i}$ has birational transform $D_{i}$. Let $\bar{p}_{i}$ be the corresponding interior special points. Then, by construction, the intersection graph of the interior square negative curves is $\Gamma_{2}^{(n, m)}$. As $D_{2}^{2}=3, m \geq 2$. If $m>2, \psi$ factors through the blow down of a tree of $(m-2)$ curves in $\operatorname{Ex}(\pi)$ with center $p_{2}$, as $D_{2}^{2}=3$. This tree is uniquely given by definition of $\Gamma_{2}^{(n, m)}$. So we can assume $m=2$. Similarly, $n=0$. Thus, the graph given by interiror square negative curves on $\tilde{Y}$ is given by $\Gamma_{2}^{(0,2)}$, see Figure 16 . Similarly $\psi$ is the $(4,0)$ blow-up of $Y$ in $\left(p_{1}, p_{2}\right)$. The augmented curve structure $\Gamma_{Y}$ is given in Figure 17. Note that $\Gamma_{Y}$ is tamely degenerate. 


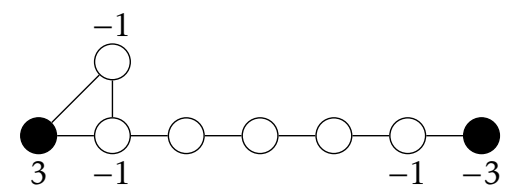

Figure 17. The augmented curve structure $\Gamma_{Y}^{a}$ of Example 4.22. All unlabelled vertices in $\Gamma_{Y}$ have self-intersection -2 .

Lemma 4.23. Let $Y$ be a component of $Y_{c} \in \operatorname{Mod}_{2}$. Suppose $\Gamma_{Y}$ is of type $d_{2}$ and very degenerate. Write $D=D_{1}+D_{2}$. Then for each component $D_{i}$ of $D$ there is a unique vertex $v_{D_{i}}$ of $\Gamma_{Y}$ with $D_{i} \cdot v_{D_{i}}>0$.

Proof. The claim is trivial if $\left|\Gamma_{Y}\right|=1$. If $\left|\Gamma_{Y}\right|>1$, we find morphisms $\pi: \tilde{Y} \rightarrow \bigcup_{2}$ and $\psi: \tilde{Y} \rightarrow Y$ as in the proof of Proposition 4.19. By construction of $\tilde{Y}$, the intersection graph $\Gamma_{2}^{\mathrm{n}}$ of $\tilde{Y}$ has precisely one branch consisting of a single vertex $w$. It follows easily from the definition of regularity that $\pi$ contracts $w$. The claim follows.

Let $\Gamma_{Y}$ be a curve structure. Consider a map

$$
f: \Gamma_{Y} \rightarrow \mathbb{Z}
$$

By abuse of notation we mean here that the map is defined on the underlying set of $\Gamma_{Y}$, i.e. its vertices.

This defines a divisor

$$
\Gamma_{f}=\sum_{v \in \Gamma_{Y}} f(v) C_{v}
$$

Rephrasing Lemma 4.4, we obtain the following lemma.

Lemma 4.24. Let $\Gamma_{f} \cdot v>0$ for all $v \in \Gamma_{Y}$. Suppose there is an exceptional vertex $v_{0} \in \Gamma_{Y}$ and suppose $f\left(v_{0}\right)>0$. Assume the leg $L\left(v_{0}\right)$ defined by $v_{0}$ is given by the graph $\left(v_{0}, v_{1}, v_{2}, \ldots, v_{n}\right)$. Then $f\left(v_{i+1}\right)>f\left(v_{i}\right)$ for $i=0, \ldots, n-1$.

Lemma 4.25. Let $\Gamma_{Y}$ be of type $d_{2}$. Let $\Gamma_{f}$ be a divisor defined by a map $f: \Gamma_{Y} \rightarrow \mathbb{Z}$. Suppose $\Gamma_{f} \cdot v>0$ for all $v \in \Gamma_{Y}$ and assume that $f\left(v_{e}\right)>0$ for all exceptional vertices $v_{e}$ of $\Gamma_{Y}$ or, if there is no exceptional vertex, $f(c)>0$ for the unique vertex meeting both components of $D$. Then $\Gamma_{f}$ is an ample divisor.

Proof. If $\Gamma_{Y}$ does not have an exceptional vertex, it is degenerate. It follows from the description of possible degenerate curve structures without exceptional vertices, compare Figure 15, that all $f(v)$ are strictly positive. The same statement holds if $Y$ has an exceptional vertex. This follows from Lemma 4.24. We next claim that for any curve $C$ not in $C(Y)$ there exists some $C_{v}, v \in \Gamma_{Y}$ such that $C \cdot C_{v}>0$. To see this, let $A$ be an ample divisor on $Y$. Then $A \cdot C>0$. As $C(Y)$ is a basis of $\operatorname{Pic}(Y)_{\mathbb{Q}}$, we can, replacing $A$ by a multiple if necessary, write $A=\sum_{C_{v} \in C(Y)} \beta_{v} C_{v}$ with $\beta_{v} \in \mathbb{Z}$. Then $C \cdot C_{v}>0$ for some $v \in C(Y)$, as else $A \cdot C=0$. It follows that $\Gamma_{f} \cdot C>0$ for all integral curves, so $\Gamma_{f}$ is positive on $\mathrm{NE}(Y)$, as the latter is finitely polyhedral by Corollary 4.7. By Kleiman's criterion, $\Gamma_{f}$ is ample.

Proposition 4.26. Let $Y$ be a component of a surface $Y_{c} \in \operatorname{Mod}_{2}(\mathscr{P})$. Suppose $\Gamma_{Y}$ is non-degenerate. Let $f_{0}, g_{0}$ be the exceptional vertices and let $k_{1}, k_{2}$ be non-negative integers. Then, there is a positive integer $\triangle$ such that for any e $>\Delta$, there is a map $f: \Gamma_{Y} \rightarrow \mathbb{Z}$ such that $\Gamma_{f}$ is an ample divisor with $f\left(f_{0}\right)=e+k_{1}$ and $f\left(g_{0}\right)=e+k_{2}$.

Proof. It follows from the discussion of Example 4.16 that $\Gamma_{Y}$ has a unique fork $c$. Let $\left(f_{0}, \ldots, f_{n}, c\right)$ and $\left(g_{0}, \ldots, g_{m}, c\right)$ denote the legs associated to the exceptional vertices. Let $y$ denote the unique vertex not appearing on any of the legs. We can assume

$$
k_{1}+\frac{1}{2} n(n+1) \geq k_{2}+\frac{1}{2} m(m+1) .
$$

Set $\Delta^{\prime}:=k_{1}+\frac{1}{2} n(n+1)-k_{2}-\frac{1}{2} m(m+1)+2 \max \{n, m\}+2$. Let $e$ be any integer such that $e>\Delta:=2 \Delta^{\prime}+2$. 
Define a map $f: \Gamma_{Y} \rightarrow \mathbb{Z}$ by

$$
\begin{aligned}
& f\left(f_{i}\right)=e+k_{1}+\frac{1}{2} i(i+1) \\
& f\left(g_{i}\right)=e+k_{2}+\frac{1}{2} i(i+1) \\
& f(c)=\max \left\{f\left(f_{n}\right), f\left(g_{m}\right)\right\}+\max \{n, m\}+1 \\
& f(y)=\left\lceil\frac{1}{2} e\right\rceil-1 .
\end{aligned}
$$

Note that by assumption $f\left(f_{n}\right) \geq f\left(g_{m}\right)$. Also, we have $f\left(f_{1}\right)-f\left(f_{0}\right)=1$ and

$$
f\left(f_{i-1}\right)-2 f\left(f_{i}\right)+f\left(f_{i+1}\right)=1
$$

for $1 \leq 1 \leq n-1$. Also, $f\left(f_{i}\right)-f\left(f_{i-1}\right)=i$, so

$$
-2 f\left(f_{n}\right)+f(c)+f\left(f_{n-1}\right)=\max \{n, m\}+1-n>0 .
$$

Analoguously, we find $f\left(g_{1}\right)-f\left(g_{0}\right)=1, f\left(g_{i-1}\right)-2 f\left(g_{i}\right)+f\left(g_{i+1}\right)=1$ for $1 \leq 1 \leq m-1$ and

$$
-2 f\left(g_{m}\right)+f(c)+f\left(g_{m-1}\right) \geq \max \{n, m\}+1-m>0 .
$$

We also have

$$
\begin{aligned}
-2 f(c)+f\left(f_{n}\right)+f\left(g_{m}\right)+f(y) & =-2 \max \left\{f_{n}, g_{m}\right\}-2 \max \{n, m\}-2+f\left(f_{n}\right)+f\left(g_{m}\right)+f(y) \\
& =-f\left(f_{n}\right)+f\left(g_{m}\right)+f(y)-2 \max \{n, m\}-2 \\
& =f(y)-\Delta^{\prime}>0 .
\end{aligned}
$$

Finally, $\max \left\{f\left(f_{n}\right), g\left(g_{m}\right)\right\} \geq e$, so $-2 f(y)+f(c)=-e+2+\max \left\{f_{n}, g_{m}\right\}+\max \{n, m\}+1>0$. By Lemma 4.25 , $\Gamma_{f}$ is an ample divisor with properties as desired.

Proposition 4.27. Let $Y$ be a component of a surface $Y_{c} \in \operatorname{Mod}_{2}(\mathscr{P})$. Suppose $\Gamma_{Y}$ is non-degenerate. Write $D=D_{1}+D_{2}$ for the anticanonical cycle. Assume $D_{1}^{2} \leq 0$. Let $k_{1}, k_{2}$ and $\gamma$ be integers with $k_{1}, k_{2} \geq 0$ and $\gamma>1$. Then there are positive integers $\Delta$ and $\alpha$ such that for any $e>\triangle$ divisible by $\alpha$, there is an ample divisor $A$ with $A \cdot D_{1}=e+k_{1}$ and $A \cdot D_{2}=\gamma e+k_{2}$.

Proof. Assume first that $D_{1}^{2}<0$. Take an ample divisor $L$ on $Y$ and set $M=-D_{1}^{2} L+\left(L \cdot D_{1}\right) D_{1}$. Let $C$ be an integral curve on $Y$. If $C$ is distinct form $D_{1}$ then $M \cdot C=\left(-D_{1}^{2}\right) L \cdot C+\left(L \cdot D_{1}\right) D_{1} \cdot C>0$. Also, $M \cdot D_{1}=-D_{1}^{2} L \cdot D_{1}+\left(L \cdot D_{1}\right) D_{1}^{2}=0$. If $D_{1}^{2}=0$, then set $M=D_{1}$. In any case there is a nef divisor $M$ with $M \cdot D_{2}=\alpha>0$. and $M \cdot D_{1}=0$. Now, by Proposition 4.26, there is an integer $\Delta$ and such that for any integer $e>\Delta$ there is an ample divisor $\Gamma_{f}$ such that $\Gamma_{f} \cdot D_{1}=e+k_{1}$ and $\Gamma_{f} \cdot D_{2}=e+k_{2}$. Fix $e=\alpha r$ for some integer $r>0$ such that $e>\Delta$. Then $A:=\Gamma_{f}+(\gamma-1) r M$ is ample. Also, $A \cdot D_{1}=e+k_{1}$ and $A \cdot D_{2}=k_{2}+\gamma e$, as desired.

Example 4.28. Proposition 4.27 fails if $D_{1}^{2}>0$, as the legs of the curve structure are then too short: let $D^{\prime}=D_{1}^{\prime}+D_{2}^{\prime}$ be the anticanonical divisor of $\bigsqcup_{2}, p_{i} \in D_{i}^{\prime}, i=1,2$ be the interior special points of $y_{2}$. Assume that $C$ is the (-1)-curve meeting $D_{1}^{\prime}$ and $F$ the (-2)-curve with $C \cdot F=1$. For $n \in \mathbb{N}$ let $Y(n)$ be the surface obtained from $\bigcup_{2}$ by blowing up $n$ times in $p_{2}$ and contracting $C+F$. Note that $\Gamma_{Y(n)}$ is non-degenerate of type $d_{2}$. Let $k$ and $e$ be positive integers. Suppose $A$ is an ample divisor with $A \cdot D_{1}=e$ and $A \cdot D_{2}=\gamma e+k$ with some integer $\gamma>1$, and define $f: \Gamma_{Y(n)} \rightarrow Z$ to be the map with $A=\Gamma_{f}$. Let $v_{e_{i}}$ be the exceptional vertex meeting $D_{i}$. We have $L\left(v_{e_{1}}\right)=\left(e_{1}, c\right)$ with $c$ the fork of $\Gamma_{Y(n)}$. Also, write $L\left(v_{e_{2}}\right)=\left(v_{e_{2}}, \ldots, w, c\right)$ and let $y$ be the unique vertex not on any leg. We have $f(c)=\gamma e+\alpha+\beta$ and $f(w)=\gamma e+\beta$ with $\alpha, \beta>0$ by Lemma 4.24. By ampleness $A \cdot c>0$, so $f\left(e_{1}\right)+f(w)+f(y)>2 f(c)$ and using that $A \cdot D_{1}=f\left(e_{1}\right)=e$ we obtain

$$
f(y)>(\gamma-1) e+2 \alpha+\beta .
$$


Intersecting $A$ with $y$ gives $-2 f(y)+f(c)>0$. So $0>2 f(y)-f(c)>2(\gamma-1) e+4 \alpha+2 \beta-f(c)$, implying $0>(\gamma-2) e+3 \alpha+\beta>0$, a contradiction. Hence a divisor such as $A$ cannot exist.

We note the following variant of Proposition 4.26.

Proposition 4.29. Let $Y$ be a component of a surface $Y_{c}$ in $\operatorname{Mod}_{2}(\mathscr{P})$, with $\Gamma_{Y}$ non-degenerate. Let $D=D_{1}+D_{2}$ be the anticanonical divisor. Assume $D_{1}^{2}=1$. Let $k \geq 0$ be an integer. Then there is an integer $\triangle>0$ such that for any even integer $e>\triangle$ an ample divisor $A$ on $Y$ exists with $A \cdot D_{1}=e$ and $A \cdot D_{2}=\frac{3}{2} e+k$.

Proof. Let $c$ be the fork of $\Gamma_{Y}$ and let $v_{e_{i}}$ denote the exceptional vertex with $D_{i} \cdot v_{e_{i}}=1$. Let us consider $L\left(v_{e_{2}}\right)=\left(f_{0}, \ldots, f_{n}, c\right)$. Set $\Delta=n(n+1)+6 n+2 k+10$ and denote by $y$ the unique vertex not appearing on any of the legs.

Let $e$ be any even integer such that $e>\Delta$. Define a map $f: \Gamma_{Y} \rightarrow \mathbb{Z}$ by

$$
\begin{aligned}
& f\left(v_{e_{1}}\right)=e \\
& f\left(f_{i}\right)=\frac{3}{2} e+k+\frac{1}{2} i(i+1) \\
& f(c)=f\left(f_{n}\right)+n+1 \\
& f(y)=\frac{1}{2} e+\frac{1}{2} n(n+1)+2 n+3+k .
\end{aligned}
$$

Then $-f\left(v_{e_{1}}\right)+f(c)=f\left(f_{n}\right)+n+1-e>0$. As above, we have $f\left(f_{1}\right)-f\left(f_{0}\right)=1$ and

$$
f\left(f_{i-1}\right)-2 f\left(f_{i}\right)+f\left(f_{i+1}\right)=1
$$

for $1 \leq i \leq n-1$. Moreover, $f\left(f_{i}\right)-f\left(f_{i-1}\right)=i$ for $1 \leq i \leq n-1$, so

$$
-2 f\left(f_{n}\right)+f(c)+f\left(f_{n-1}\right)=1
$$

We also have

$$
\begin{aligned}
-2 f(c)+f\left(f_{n}\right)+f\left(v_{e_{1}}\right)+f(y) & =-2 f\left(f_{n}\right)-2 n-2+f\left(f_{n}\right)+f\left(v_{e_{1}}\right)+f(y) \\
& =-f\left(f_{n}\right)+f\left(v_{e_{1}}\right)+f(y)-2 n-2 \\
& =-\frac{3}{2} e-k-\frac{1}{2} n(n+1)-2 n-2+e+f(y) \\
& =1 .
\end{aligned}
$$

Finally

$$
\begin{aligned}
-2 f(y)+f(c) & =-e-n(n+1)-4 n-6-2 k+\frac{3}{2} e+k+\frac{1}{2} n(n+1)+n+1 \\
& =\frac{1}{2} e-\frac{1}{2} n(n+1)-3 n-5-k \\
& =\frac{1}{2}(e-\Delta)>0 .
\end{aligned}
$$

Hence $\Gamma_{f}$ is an ample divisor with properties as desired.

We will also need to construct ample divisors on surfaces with degenerate curve structures. For better readability, we will treat the different cases separately.

Proposition 4.30. Let $Y$ be a component of a surface $Y_{c} \in \operatorname{Mod}_{2}(\mathscr{P})$. Suppose $\Gamma_{Y}$ is of type $d_{2}$ and tamely degenerate. Suppose there is an exceptional vertex $v_{0}$ and that e is an integer, $e>3$. Write $D=D_{1}+D_{2}$ with $D_{1}$ denoting the component of $D$ with $D_{1} \cdot v_{0}=1$. Then there is a map $f: \Gamma_{Y} \rightarrow \mathbb{Z}$ such that $\Gamma_{f}$ is an ample divisor with degree $e+m$ on $D_{2}$ and degree e on $D_{1}$ for some integer $m \geq 0$ independent of e. If $\Gamma_{Y}$ does not have an exceptional vertex, then there is a map $f: \Gamma_{Y} \rightarrow \mathbb{Z}$ such that $\Gamma_{f}$ is an ample divisor with degree e on all components of $D$ that are met by a unique element of $\Gamma_{Y}$ and $e+m$ on the remaining components for some $m \geq 0$ independent of $e$. 
Proof. Write $L\left(v_{0}\right)=\left(v_{0}, \ldots, v_{n}\right)$. Let $w$ be the unique element in $\Gamma_{Y} \backslash L\left(v_{0}\right)$ which exists by regularity. Let $e \geq 2$. Define $f: \Gamma_{Y} \rightarrow \mathbb{Z}$ by setting $f\left(v_{i}\right)=e+\frac{1}{2} i(i+1)$ and $f(w)=n+1$. Then $\Gamma_{f} \cdot v_{i}=1$ for all $i$ and also $\Gamma_{f} \cdot w>0$. It follows from Lemma 4.25 that $\Gamma_{f}$ is an ample divisor with degree $e$ on $D_{1}$ and degree $e+\left(D_{2} \cdot w\right)(n+1)+\frac{1}{2} n(n+1)$ on $D_{2}$. Since $D_{2} \cdot w>0$ we obtain the claim by setting $m=\left(D_{2} \cdot w\right)(n+1)+\frac{1}{2} n(n+1)$. In the case without exceptional vertex, $\Gamma_{Y}$ is given by $\left\{v_{0}, v_{1}\right\}$ with $v_{0}^{2}=0$, $v_{1}^{2} \geq-2$. The divisor $3 v_{0}+v_{1}$ has the required properties.

Let $\Gamma_{Y}$ be of type $d_{2}$, very degenerate and with no exceptional vertex. Then $\Gamma_{Y}$ is a singleton given by a vertex $v$. Conversely, if $Y$ is a component of a surface $Y_{c} \in \operatorname{Mod}_{2}(\mathscr{P})$ with $\Gamma_{Y}$ a singleton, then $\Gamma_{Y}$ is of type $d_{2}$, very degenerate and with no exceptional vertex. For later use we note the following obvious statement.

Proposition 4.31. Let $Y$ be a component of a surface $Y_{c} \in \operatorname{Mod}_{2}(\mathscr{P})$. Suppose $\Gamma_{Y}$ is a singleton given by a vertex $v$. Then, after relabelling $D$, we have $D_{1} \cdot v=1$ and $D_{2} \cdot v=2$. Then, for any $e>0, v \mapsto e$ defines an ample divisor with degree e on $D_{1}$ and $2 e$ on $D_{2}$ and any ample divisor is of this form.

Proposition 4.32. Let $Y$ be a component of a surface $Y_{c} \in \operatorname{Mod}_{2}(\mathscr{P})$. Suppose $\Gamma_{Y}$ is very degenerate. Suppose there is an exceptional vertex $v_{0}$ and let $e>2$ be an integer. Write $D=D_{1}+D_{2}$ with $D_{1}$ denoting the component of $D$ with $D_{1} \cdot v_{0}=1$. Then there is a map $f: \Gamma_{Y} \rightarrow \mathbb{Z}$ such that $\Gamma_{f}$ is an ample divisor with degree $2 e+m$ on $D_{2}$ and degree e on $D_{1}$ for some integer $m>0$ independent of $e$.

Proof. As $\Gamma_{Y}$ is very degenerate and hence degenerate, $L\left(v_{0}\right)=\Gamma_{Y}$. Write $L\left(v_{0}\right)=\left(v_{0}, v_{1}, \ldots, v_{n}\right)$ and setting $f\left(v_{i}\right)=e+\frac{1}{2} i(i+1)$ defines an ample divisor (note that $\left.v_{n}^{2}=0\right)$ with degree $2\left(e+\frac{1}{2} n(n+1)\right)$ on $D_{2}$ and $e$ on $D_{1}$.

\section{Projective Models}

In this section we analyse the elements in the set $\mathrm{PMod}_{2}$ in terms of curve structures. This analysis will be divided into two cases, namely models of type $\mathscr{P}$ and of type $\mathscr{T}$. We shall, in particular, prove projectivity criteria.

\subsection{Models of class $\mathscr{T}$}

Recall from Example 2.30 that $Y_{\mathscr{T}}$ has a component with normalisation $\bigcup_{4}$. Denote this component by $\left(Y_{\mathscr{T}}\right)_{\omega}$. Let $Y_{c} \in \operatorname{Mod}_{2}(\mathscr{T})$. There is a sequence of type I flops to $Y_{\mathscr{T}}$. Let $Y_{\omega}$ be the image of $\left(Y_{\mathscr{T}}\right)_{\omega}$ under the induced birational map. It is independent of the chosen sequence. We call $Y_{\omega}$ the special component of $Y_{c}$.

Proposition 5.1. Let $Y_{c} \in \operatorname{Mod}_{2}(\mathscr{T}), Y_{\omega}$ the special component. Let $D_{\omega}$ be the smooth component of the boundary curve of $Y_{\omega}$. Let $D_{1}$ and $D_{2}$ be the disjoint curves in the preimage of $D_{\omega}$ under the normalisation $\pi: Y_{\omega}^{v} \rightarrow Y_{\omega}$. Then $Y_{c}$ is projective if and only if $D_{1}^{2}=D_{2}^{2}=-1$.

Proof. Let $Y_{c}=Y_{1} \cup Y_{2} \cup Y_{3}$ and suppose $Y_{\omega}=Y_{2}$. We begin by showing that $Y_{c} \in \operatorname{PMod}_{2}(\mathscr{T})$ if and only if $Y_{\omega}$ is projective. Projectivity of $Y_{c}$ clearly implies projectivity of $Y_{w}$, so we only need to show the opposite implication. So suppose $Y_{\omega}$ is projective. Because $Y_{c}$ is in $\operatorname{Mod}_{2}$ and has dual intersection complex $\mathscr{T}$, the remaining two components of $Y_{c}$ are obtained by blow-ups and blow-downs of $\bigcup_{1}$ in the interior special point, and thus are projective. Hence there are ample line bundle $A_{i}$ on $Y_{i}$ for $i=1,2,3$. The components $Y_{1}$ and $Y_{2}$ are glued by identifying a curve $D_{12} \subset Y_{1}$ with a curve $D_{21} \subset Y_{2}$. Similarly, $Y_{3}$ and $Y_{2}$ are glued by identifying a curve $D_{32} \subset Y_{3}$ with a curve $D_{23} \subset Y_{2}$. Replacing the $A_{i}$ by suitable multiples, we can assume that $A_{1} \cdot D_{12}=A_{2} \cdot D_{21}$ and $A_{2} \cdot D_{23}=A_{3} \cdot D_{32}$. Because $Y_{c}$ has trivial Carlson extension, one obtains an ample bundle on $Y_{c}$ by Lemma 2.10 and [Laz04, Proposition 1.2.16], implying that $Y_{c}$ is projective.

We now show that projectivity of $Y_{w}$ is equivalent to the numerical condition stated in the proposition. Suppose first that $D_{1}^{2}=D_{2}^{2}=-1$. One can, similar as in the proof of Proposition 2.28, find an ample divisor 
on $Y_{\omega}$. Indeed, take an ample divisor $L^{\prime}=L_{\omega}^{\prime}$ on the normalisation $Y_{\omega}^{v}$. Suppose without loss of generality $L^{\prime} \cdot D_{1} \geq L^{\prime} \cdot D_{2}$. Set $L^{\prime \prime}=\left(L^{\prime} \cdot D_{2}\right) L^{\prime}$. Set $M=L^{\prime}+\left(L^{\prime} \cdot D_{1}\right) D_{1}$. Then $M \cdot C \geq 0$ for all curves $C$ on $Y_{\omega}^{v}$ with equality if and only if $C=D_{1}$. Write $n=L^{\prime} \cdot D_{1}-L^{\prime} \cdot D_{2}$. Note that $D_{1} \cdot D_{2}=0$. Then $L_{\omega}=L^{\prime \prime}+n M$ is ample by the Nakai-Moishezon criterion and

$$
\begin{aligned}
L_{\omega} \cdot D_{2} & =L^{\prime \prime} \cdot D_{2}+n M \cdot D_{2} \\
& =\left(L^{\prime} \cdot D_{2}\right)\left(L^{\prime} \cdot D_{2}\right)+\left(L^{\prime} \cdot D_{1}\right)\left(L^{\prime} \cdot D_{2}\right)-\left(L^{\prime} \cdot D_{2}\right)\left(L^{\prime} \cdot D_{2}\right) \\
& =L^{\prime \prime} \cdot D_{1}+n M \cdot D_{1} \\
& =L_{\omega} \cdot D_{1} .
\end{aligned}
$$

Hence $L_{\omega}$ induces an ample divisor on $Y_{w}$.

We now show that the condition on the intersection numbers is also necessary. So suppose that $D_{1}^{2}<-1$. Note that $D_{2}^{2}=-2-D_{1}^{2}$. Suppose $L^{\prime}$ is an ample line bundle on $Y_{\omega}$. The normalisation $v: Y_{\omega}^{v} \rightarrow Y_{\omega}$ is finite, so $L=v^{*} L^{\prime}$ is ample.

By Proposition 4.17, replacing $L$ by a suitable multiple we can write

$$
L=\sum_{v \in C(Y)} a_{v} C_{v}
$$

with $a_{v} \in \mathbb{Z}$.

As $L$ is ample, the coefficients of vertices meeting the anticanonical divisor are strictly positive. This implies $a_{v}>0$ for all $v$ by Lemma 4.24, as either $v$ is contained in the leg defined by some exceptional vertex or meets a component of the boundary. By the condition on the self-intersection of $D_{1}$ the curve structure $\Gamma_{Y}$ is degenerate: there is an exceptional vertex $e$ with $e_{1} \cdot D_{1}=1$ and $L(e)$ ends in a vertex $w$ with $w \cdot D_{2}=1$. By Lemma 4.24 ,

$$
L \cdot D_{2}>a_{w}>a_{e}=L \cdot D_{1}
$$

so $Y_{\omega}$ cannot be projective.

In particular, the proposition says that $Y_{c} \in \operatorname{Mod}_{2}$ is in $\operatorname{PMod}_{2}(\mathscr{T})$ if and only if there is a sequence of type I flops

$$
Y_{c} \rightarrow \cdots \rightarrow Y_{i} \rightarrow \cdots \rightarrow Y_{\mathscr{T}},
$$

such that any flopping curve meets the nodal components of the double locus on the special component of $Y_{i}$.

\subsection{Models of class $\mathscr{P}$}

Proposition 5.2. Let $Y_{c} \in \operatorname{Mod}_{2}(\mathscr{P})$ and write $Y_{c}=Y_{1} \cup Y_{2} \cup Y_{3}$.

(i) If $\Gamma_{Y_{i}}$ is degenerate for all $i=1,2,3$, then $Y_{c}$ is not projective.

(ii) Suppose $\Gamma_{Y_{i}}$ is regular for all $i$. If there is a component $Y_{i}$ such that $\Gamma_{Y_{i}}$ is non-degenerate, then $Y_{c}$ is projective.

Proof. Suppose first that the curve structures $\Gamma_{Y_{i}}$ are all degenerate. We show that this cannot happen if $Y_{c}$ is projective. So let $A$ be an ample divisor on $Y_{c}$. Denote the restriction to $Y_{i}$ by $A_{i}$. The set of underlying curves of $\Gamma_{Y_{i}}$ is a basis of $\operatorname{Pic}\left(Y_{i}\right)_{\mathbb{Q}}$ and thus after replacing $A$ by a suitable multiple, we write $A_{i}=\Gamma_{f_{i}}$ for a suitable function $f_{i}: \Gamma_{Y_{i}} \rightarrow \mathbb{Z}$. Note that at least one of the $Y_{i}$, say $Y_{1}$, has an exceptional vertex $v_{e}$. Let the leg of $v_{e}$ be given by $L\left(v_{e}\right)=\left(v_{e}, \ldots, v_{n}\right)$. Suppose the component of the anticanonical divisor met by $v_{e}$ is $D_{13}$ while $v_{n}$ meets $D_{12}$. Necessarily $f\left(v_{e}\right)>0$, and thus we have $f\left(v_{n}\right)>f\left(v_{e}\right)$ by Lemma 4.24 . In particular, $A_{1} \cdot D_{12}>A_{1} \cdot D_{13}$. Now consider $Y_{2}$. If $\Gamma_{Y_{2}}$ has an exceptional vertex, we find $A_{2} \cdot D_{23}>A_{2} \cdot D_{21}$ by the same reasoning. If $\Gamma_{Y_{2}}$ has no exceptional vertex and $\left|\Gamma_{Y_{2}}\right|>1$, then $\Gamma_{Y_{2}}$ has two elements, say $v_{1}$ and $v_{2}$ with indices chosen such that $v_{1}^{2}=0, v_{1} \cdot v_{2}=1, D_{21} \cdot v_{2}=0$ and $D_{21} \cdot v_{1}=1$. Write $A_{2}=a_{1} v_{1}+a_{2} v_{2}$. 
Then $0<A_{2} \cdot v_{1}=a_{2}$ and $0<A_{2} \cdot D_{21}=a_{1}$. So $A_{2} \cdot D_{23} \geq A_{2} \cdot D_{21}$. If $\left|\Gamma_{Y_{2}}\right|=1$, we have $A_{2} \cdot D_{23}=2 A_{2} \cdot D_{21}$ by virtue of Proposition 4.31 .

The same reasoning applied to $Y_{3}$ yields the chain of inequalites

$$
A_{2} \cdot D_{23} \geq A_{2} \cdot D_{21}=A_{1} \cdot D_{12}>A_{1} \cdot D_{13}=A_{3} \cdot D_{31} \geq A_{3} \cdot D_{32}=A_{2} \cdot D_{23},
$$

where the equalities come from the gluing condition. Hence $Y_{c}$ is not projective.

Conversely, suppose the curve structure $\Gamma_{Y_{1}}$ is non-degenerate. For large enough $e$, there is an ample divisor $A_{2}$ on $Y_{2}$ with $\operatorname{deg} A_{2 \mid D_{21}}=e$ and $\operatorname{deg} A_{2 \mid D_{23}}=e+k_{1}$, with $k_{1} \geq 0$ and independent of $e$, by Proposition 4.26 if $\Gamma_{Y_{2}}$ is non-degenerate or Proposition 4.30 in the degenerate case. Similarly, maybe increasing $e$, one finds an ample divisor $A_{3}$ on $Y_{3}$ with $A_{3} \cdot D_{32}=e+k_{1}$ and $A_{3} \cdot D_{31}=e+k_{2}, k_{2} \geq 0$. Then, maybe again increasing $e$, there is an ample divisor $A_{1}$ on $Y_{1}$ with $A_{1} \cdot D_{13}=e+k_{2}$ and $A_{1} \cdot D_{12}=e$, as $\Gamma_{Y_{1}}$ is non-degenerate and hence Proposition 4.26 applies. Hence $Y$ carries an ample bundle and thus is projective.

Recall that if $\Gamma_{Y_{i}}$ is a curve structure of type $d_{2}$ that is very degenerate and $D_{i j}$ is a component of the anticanonical cycle, then there is a unique vertex $v_{D_{i j}} \in \Gamma_{Y_{i}}$ such that $v_{D_{i j}} \cdot D_{i j} \neq 0$, by Lemma 4.23. Note that if $Y=Y_{1} \cup Y_{2} \cup Y_{3}$ is of class $\mathscr{P}$ such that $\Gamma_{Y_{1}}$ is non-degenerate and $\Gamma_{Y_{i}}$ is very degenerate for $i=2,3$, then there exists an $i$ such that $v_{D_{i 1}} \cdot D_{i 1}=2$.

Proposition 5.3. Let $Y_{c} \in \operatorname{Mod}_{2}(\mathscr{P})$ and write $Y_{c}=Y_{1} \cup Y_{2} \cup Y_{3}$. Suppose $\Gamma_{Y_{1}}$ is non-degenerate and $\Gamma_{Y_{i}}$ is very degenerate for $i=2,3$.

(i) If $v_{D_{i 1}} \cdot D_{i 1}=2$ for $i=2,3$, then $Y_{c}$ is projective.

(ii) If $v_{D_{i 1}} \cdot D_{i 1}=1$ for exactly one $i \in\{2,3\}$, then $Y_{c}$ is projective if and only if $D_{1 i}^{2} \leq 0$.

Proof. We begin by proving the first claim. By Propositions 4.31 and 4.32, given any sufficiently large $e$, there are ample divisors $A_{2}$ and $A_{3}$ on $Y_{2}$ and $Y_{3}$ with $A_{2} \cdot D_{23}=A_{3} \cdot D_{32}=e$ and $A_{i} \cdot D_{i 1}=2 e+n_{i}, i=2$, 3, with $n_{i} \geq 0$ and $n_{i}$ independent of $e$. As $Y_{1}$ is non-degenerate, after maybe increasing $e$, by Proposition 4.26, there is an ample divisor $A_{1}$ on $Y_{1}$ with $A \cdot D_{1 i}=2 e+n_{i}$, hence the $A_{i}$ 's glue to an ample divisor on $Y_{c}$.

In the second case, we can assume $v_{D_{21}} \cdot D_{21}=1$. If $D_{12}^{2}>0$, then in fact $D_{12}^{2}=1$, as $Y_{1}$ is non-degenerate. We want to show that there is no ample bundle on $Y_{c}$. To get a contradiction, suppose $A$ is ample on $Y_{c}$. Write $A_{i}$ for the restriction to $Y_{i}$. Write $A_{2} \cdot D_{21}=e>0$. Note that $\left|\Gamma_{Y_{2}}\right|>1$. So by Proposition 4.32 $A_{2} \cdot D_{23}=2 e+n_{1}$ for some number $n_{1}>0$.

As $v_{D_{31}} \cdot D_{31}=2$, by the same results and the gluing condition, we have $A_{3} \cdot D_{32}=2 e+n_{1}$ and $A_{3} \cdot D_{31}=4 e+n_{2}$ for some $n_{2}>0$. It follows that $A_{1} \cdot D_{12}=e$ and $A_{1} \cdot D_{13}=4 e+n_{2}$ by the gluing condition. But then $A_{1}$ cannot be ample by Example 4.28.

If $D_{12}^{2} \leq 0$, we can, by Lemma 4.24 and Proposition 4.31, find an ample divisor on $A_{2}$ on $Y_{2}$ with $A_{2} \cdot D_{21}=e$ and $A_{2} \cdot D_{23}=2 e+n_{1}$ with $n_{1}$ independent of $e$ for any $e$ sufficiently large. Similarly, we find an ample $A_{3}$ on $Y_{3}$ with $A_{3} \cdot D_{32}=2 e+n_{1}$ and $A_{3} \cdot D_{31}=4 e+n_{2}, n_{2}$ independent of $e$. By Proposition 4.27, there is a ample $A_{1}$ on $Y_{1}$ with $A_{1} \cdot D_{12}=e$ and $A_{1} \cdot D_{13}=4 e+n_{2}$. By construction, the $A_{i}$ glue to an ample bundle $A$ on $Y_{c}$, so $Y_{c}$ is projective.

The following proposition is proven by the same reasoning as Proposition 5.3.

Proposition 5.4. Let $Y_{c} \in \operatorname{Mod}_{2}(\mathscr{P})$ and write $Y_{c}=Y_{1} \cup Y_{2} \cup Y_{3}$. Suppose $\Gamma_{Y_{1}}$ is non-degenerate, $\Gamma_{Y_{2}}$ is very degenerate and $\Gamma_{Y_{3}}$ is tamely degenerate.

(i) If $v_{D_{21}} \cdot D_{21}=1$ then $Y_{c}$ is projective if and only if $D_{12}^{2} \leq 0$.

(ii) If $v_{D_{21}} \cdot D_{21}=2$ then $Y_{c}$ is projective if and only if $D_{13}^{2} \leq 0$.

Proposition 5.5. Let $Y_{c} \in \operatorname{Mod}_{2}(\mathscr{P})$ and write $Y_{c}=Y_{1} \cup Y_{2} \cup Y_{3}$. Suppose $\Gamma_{Y_{1}}$ is very degenerate and $\Gamma_{Y_{i}}$ is non-degenerate for $i=2,3$. Also, assume $v_{D_{12}} \cdot D_{12}=2$. Then $Y_{c}$ is projective. 
Proof. Note that by non-degeneracy, $D_{31}^{2} \leq 1$ and $D_{23}^{2} \leq 1$. If $D_{31}^{2} \leq 0$ or $D_{23}^{2} \leq 0$ then $Y_{c}$ is projective: this follows as in the proof above. If $D_{31}^{2}=1$ and $D_{23}^{2}=1$, the intersection numbers and the conditions on degeneracy and regularity fix the components, so there is only one such surface. It follows from Propositon 4.29 that for sufficiently large $e$ there is an ample divisor $A_{3}$ on $Y_{3}$ with $A_{3} \cdot D_{31}=e$ and $A_{3} \cdot D_{32}=\frac{3}{2} e$.

We can take $e$ to be divisible by 4 . Since $D_{31}^{2}=1, \Gamma_{Y_{1}}$ has an exceptional vertex $v_{0}$. Let us consider $L\left(v_{0}\right)=\left(v_{0}, v_{1}, \ldots, v_{n}\right)$ the associated leg. Then $v_{n}^{2}=0$ as $\Gamma_{Y_{3}}$ is very degenerate. So $C_{v_{n}}$ is a nef divisor on $Y_{1}$. By Proposition 4.32, there is an ample divisor $A_{1}^{\prime}$ on $Y_{1}$ with $A_{1}^{\prime} \cdot D_{13}=e$ and $A_{1}^{\prime} \cdot D_{12}=2 e+k$, with $k$ independent of $e$. Set $A_{1}=A_{1}^{\prime}+\frac{1}{4} e C_{v_{n}}$. This is an ample divisor with $A_{1} \cdot D_{13}=e$ and $A_{1} \cdot D_{12}=\frac{9}{4} e+k$. By Proposition 4.29, there is an ample divisor $A_{2}$ on $Y_{2}$ with $A_{2} \cdot D_{23}=\frac{3}{2} e$ and $A_{2} \cdot D_{21}=\frac{9}{4} e+k$. Hence the $A_{i}$ glue to an ample divisor on $Y$.

\section{Flops, Birational Automorphisms and Orbits}

In this section we analyze possible flops of models of the DNV family of degree 2. For this we use augmented curve structures. Finally, we study the action of the birational automorphism group on the Mori fan where we determine the possible orbit lengths of maximal cones in this fan.

\subsection{Flops}

We first recapitulate Lemma 1.29 from [GHKS], whose proof is an elementary but lengthy computation.

Lemma 6.1 (cf. [GHKS]). Let $\mathcal{Y} \rightarrow S$ be a model of the Dolgachev-Nikulin-Voisin family of degree $2 d$, and let $\pi: \mathcal{Y} \rightarrow \mathcal{Z}$ be a birational contraction (over $S$ ) whose exceptional locus $\operatorname{Ex}(\pi)$ intersects $\mathcal{Y}_{c}$ in a one dimensional scheme. Let $v: \mathcal{Y}_{c}^{v} \rightarrow \mathcal{Y}_{c}$ be the normalisation. Then each connected component $C$ of $\operatorname{Ex}(\pi) \cap \mathcal{Y}_{c}$ is one of the following:

(i) A non-singular irreducible component of the double curve of $\mathcal{Y}_{c}$. Necessarily $v^{-1}(C)$ consists of two copies of $\mathbb{P}^{1}$, each with self intersection -1 in $\mathcal{Y}_{c}^{v}$.

(ii) A tree of rational curves contained in an irreducible component of $\mathcal{Y}_{c}$. This tree is of the form $v\left(C^{\prime}\right)$, where $C^{\prime}$ is a connected tree of rational curves contained in one connected component of $\mathcal{Y}_{c}^{v}$, intersecting its boundary transversally in one point.

(iii) A tree of rational curves in an irreducible component of $\mathcal{Y}_{c}$, disjoint from the double curve.

(iv) $v^{-1}(C)$ consists of two connected components, each intersecting the boundary of the connected component of $\mathcal{Y}_{c}^{v}$ containing it transversally in one point. These two intersection points are identified under $v$.

Remark 6.2. In (iv), the two connected components can lie in one or two components of $\mathcal{Y}_{c}$.

The lemma implies the following description of flopping contractions.

Proposition 6.3. Let $\mathcal{Y} \rightarrow S$ be a model of the Dolgachev-Nikulin-Voisin family of degree $2 d$, and let $\pi: \mathcal{Y} \rightarrow \mathcal{Z}$ be a birational contraction (over $S$ ) with $\operatorname{codim} \operatorname{Ex}(\pi)=2$, i.e. a flopping contraction. Let $C$ be a connected component of $\operatorname{Ex}(\pi)$. Let $v: \mathcal{Y}_{c}^{v} \rightarrow \mathcal{Y}_{c}$ be the normalisation. Then $v^{-1}(C)$ is an integral $(-1)$-curve if $C$ is not contained in the singular locus of $\mathcal{Y}_{c}$ and a disjoint union of two integral $(-1)$-curves if $C$ is contained in the singular locus.

Proof. First, note that $\operatorname{Ex}(\pi) \subset \mathcal{Y}_{c}$ and hence the connected components of $\operatorname{Ex}(\pi)$ are as in Lemma 6.1. In particular, one of the cases applies to $C$. In case (i) of Lemma 6.1 there is nothing to show. Suppose we are in case (ii) of the Lemma. Let $Y_{i}$ be the component of the central fibre containing $C$. Then there is an induced contraction $Y_{i}^{v} \rightarrow Z_{i}^{v}$ with $Z_{i}^{v}$ the normalisation of a component of the central fibre of $\mathcal{Z}$. The intersection matrix of the curves contracted by $Y_{i}^{v} \rightarrow Z_{i}^{v}$ is negative definite, see e.g. [KM98, Lemma 3.40]. In particular, if $C$ is reducible there is an irreducible component $C_{0} \subset C$ with $C_{0}^{2}=-2$. As $C_{0}$ does not meet the boundary, we can extend it trivially to a divisor $L_{c}$ on $\mathcal{Y}_{c}$ and then find, by maximality of the DNV 
family, a divisor $L$ on $\mathcal{Y}$ restricting to $L_{c}$. Let $F$ be a nef divisor inducing the flopping contraction $\pi$, with restriction $F_{c}$ to $\mathcal{Y}_{c}$. As $C$ is contracted, $F_{c} \cdot L_{c}=0$. Also, $L_{c}^{2}=-2$. Let $L_{\eta}, F_{\eta}$ denote the restrictions to the generic fibre. We note that the intersection numbers on $\mathcal{Y}_{c}$ can be calculated on the normalisation $\mathcal{Y}_{c}^{v}$, see e.g. [Bad01, Corollary 1.11]. Because $\mathcal{Y} \rightarrow S$ is flat, we have $L_{\eta}^{2}=L_{c}^{2}$ and $F_{\eta} \cdot L_{\eta}=F_{c} \cdot L_{c}$, by constancy of the Euler characteristic, see [Kol95, Proposition VI.2.9]. By a standard argument for K3 surfaces, either $L_{\eta}$ or $-L_{\eta}$ is effective, see [Huy16, Chapter 2, $\S 1.4$ ]. In any case, there is an integral curve on $Y_{\eta}$ contracted by $F_{\eta}$. But $\pi$ is a flopping contraction, so this is a contradiction. Hence $C$ is irreducible. As $C$ meets the double locus and and $C^{2}<0$, it is necessarily a $(-1)$-curve. In the situation of (iii) of the lemma, there is again a (-2)-curve on some component. In case (iv) one can lift the bundle given by the two irreducible $(-1)$-curves and conclude as above, as the self intersection is again -2 .

Remark 6.4. In our case $d=1$ the following holds: If $\phi: \mathcal{Y} \rightarrow \mathcal{Y}^{+}$is a flop defined by a flopping contraction $\pi: \mathcal{Y} \rightarrow \mathcal{Z}$, then the dual intersection complexes of $\mathcal{Y}$ and $\mathcal{Y}^{+}$are the same if and only if no component of $\operatorname{Ex}(\phi)$ is contained in the singular locus of $\mathcal{Y}_{c}$. This follows from the fact that any component of the singular locus meets any other such component in a triple point.

Remark 6.5. We include some remarks on terminology and on factorisation of maps into flops. We also fix some notation which we will use throughout the paper. Let $\phi: \mathcal{Y} \rightarrow \mathcal{Y}^{\prime}$ be a birational map between two models of the DNV family that is not an isomorphism. Then it is an isomorphism in codimension 1 . Let $F^{\prime}$ be an effective ample divisor on $\mathcal{Y}^{\prime}$ and let $F$ be its birational transform on $\mathcal{Y}$. Then there is a sequence of $F$-flops factoring $\phi$, i.e. there is a sequence

$$
\mathcal{Y} \rightarrow \mathcal{Y}_{1} \rightarrow \cdots \rightarrow \mathcal{Y}_{i} \rightarrow \cdots \rightarrow \mathcal{Y}_{n} \cong \mathcal{Y}^{\prime}
$$

such that each $\mathcal{Y}_{i} \rightarrow \mathcal{Y}_{i+1}$ is the flop defined by an $\tilde{F}$-flopping contraction where $\tilde{F}$ is the birational transform of $F$ under $\phi$, by Remark 3.5. We shall write $F_{k}$ for this birational transform of $F$ under $\mathcal{Y} \rightarrow \mathcal{Y}_{k}$ and $\phi_{k}: \mathcal{Y}_{k} \rightarrow \mathcal{Z}_{k}$ for the flopping contraction defining $\psi_{k}: \mathcal{Y}_{k} \rightarrow \mathcal{Y}_{k+1}$. We also write $C_{k}$ for the birational transform of $C$ under $\prod_{i=0}^{k} \phi_{i}$ if $C$ is not contracted under this map, and similar for components $Y_{i}$. We further employ a similar notation for the double curves $D_{i j} \subset Y_{i}$, so $\left(D_{i j}\right)_{k}$ is the birational transform of $Y_{i} \cap Y_{j}$ considered as a curve on $\left(Y_{i}\right)_{k}$. If we consider several maps at once, we will decorate the notation accordingly, i.e. here we would write $C_{k}^{\phi},\left(D_{j j}\right)_{k}^{\phi}$ etc. Also, note that if $C$ is a curve in $\mathcal{Y}_{k}$ with $F_{k} \cdot C>0$ and $C$ is disjoint from $\operatorname{Ex} \phi_{k}$, then $F_{k+1} \cdot\left(\psi_{k}\right)_{*} C>0$. Finally, given an $F$-flop

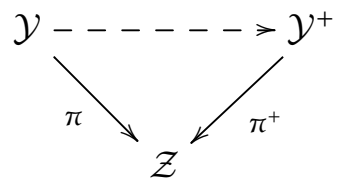

a curve in $\operatorname{Ex}(\pi)$ will be called a flopping curve and a curve in $\operatorname{Ex}\left(\pi^{+}\right)$will be called a flopped curve .

We want to classify the flopping contractions of a Kulikov model $\mathcal{Y} \rightarrow S$ of the Dolgachev-Nikulin-Voisin family of degree 2 by their exceptional loci. The next proposition shows that an elementary modification connecting central fibers of projective models lifts to a flop of the models.

Proposition 6.6. Let $\mathcal{Y}$ and $\mathcal{Y}^{\prime}$ be models of the DNV family of degree 2, with central fibres $\mathcal{Y}_{c}$ and $\mathcal{Y}_{c}^{\prime}$ respectively. Suppose that there exists a type I or type II elementary modification $Y_{c} \rightarrow Y_{c}^{\prime}$ contracting a curve $C$. Then there is a flopping contraction $\pi$ contracting precisely $C$, inducing a flop $\mathcal{Y} \rightarrow \mathcal{Y}^{\prime}$.

Proof. Let $\mathcal{X}$ and $\mathcal{X}^{\prime}$ be the maximal analytic smoothings of $\mathcal{Y}_{c}$ and $\mathcal{Y}_{c}^{\prime}$. Both are projective because the central fibres are and every line bundle of $\mathcal{Y}_{c}$ extends to $\mathcal{X}$ by maximality, and similarly for $\mathcal{Y}_{c}^{\prime}$. It follows from [KM98, Theorem 6.38] that there is a flop $\psi^{\prime}: \mathcal{X} \rightarrow \mathcal{X}^{\prime}$ given by some divisor $F$, with restriction $F_{c}$ on $\mathcal{Y}_{c}$. The curve $C$ generates an extremal ray of $\mathcal{X}$. Then $C$ also generates an extremal ray of $\mathcal{Y} \rightarrow S$ and $F_{c}$ lifts to a divisor $\mathcal{F} \in \operatorname{Pic}(\mathcal{Y} / S)$. Hence, as in the proof of Proposition 2.16, $\mathcal{F}$ defines a contraction 
$\pi: \mathcal{Y} \rightarrow \mathcal{Z}$, such that the restriction of the exceptional locus to $\mathcal{Y}_{c}$ is $C$. It is either divisorial or small. Suppose it is divisorial. Then the exceptional divisor $E$ restricts to a multiple of $C$ with multiplicity $a>0$. We shall show that the curve $C$ then leads to a contradiction of the conditions of Lemma 2.10. To see this, write $\mathcal{Y}_{c}=Y_{1} \cup Y_{2} \cup Y_{3}$. Assume first $\mathcal{Y}_{c} \in \operatorname{PMod}(\mathscr{P})$. Suppose $C$ is the exceptional locus of an elementary modification of type I. Assume the notation is such that $C \subset Y_{1}$ and $C \cdot D_{12}=1$. Write $r_{i}$ for the inclusion $Y_{i} \rightarrow \mathcal{Y}$. Then

$$
r_{1}^{*} E \cdot D_{12}=a C \cdot D_{12}=a>0 \text { and } r_{2}^{*} E \cdot D_{21}=0 .
$$

If $C$ is the exceptional locus of an elementary modification of type II, assume the normalisation of $C$ is $D_{12} \cup D_{21}$. Then

$$
r_{1}^{*} E \cdot D_{13}=a C \cdot D_{13}=2 a>0 \text { and } r_{3}^{*} E \cdot D_{31}=0 .
$$

Next assume that $\mathcal{Y}_{c} \in \operatorname{PMod}(\mathscr{T})$, and assume further that $Y_{1}$ is the special component. The proof is the same if $C$ is the exceptional locus of an elementary modification of type I. If $C$ is the exceptional locus of a type II modification, necessarily $C$ is the smooth component of the double locus on the special component $Y_{1}$. To see this, suppose this is not the case. Then we can write $D_{1 j} \cup D_{j 1}$ for the preimage of $C$ under the normalisation $v: \mathcal{Y}_{c}^{v} \rightarrow \mathcal{Y}$, with $j \in\{2,3\}$. By Proposition 6.3, $D_{1 j}^{2}=D_{j 1}^{2}=-1$. But $D_{12}^{2}+D_{21}^{2}=0$ and $D_{13}^{2}+D_{31}^{2}=0$ by Definition 2.6 and the definition of $\operatorname{PMod}(\mathscr{T})$. Hence $C$ is indeed the smooth component of the double locus on $Y_{1}$. Write $C_{1} \cup C_{2}$ for the preimage of $C$ under $v$. We have

$$
r_{1}^{*} E \cdot D_{12}=a C_{1} \cdot D_{12}+a C_{2} \cdot D_{12}=2 a>0 \text { and } r_{2}^{*} E \cdot D_{21}=0 .
$$

So $r_{c}^{*} E$ does not fullfill the degree conditions of Lemma 2.10. It follows that the extremal ray generated by $C$ defines a small contraction.

For the statement of the next corollary, recall that a surface $Y_{c} \in \operatorname{Mod}_{2}(\mathscr{T})$ has one component $Y_{\omega}$ that is not smooth, the special component.

Corollary 6.7. Let $\mathcal{Y}$ be a model of the DNV family of degree 2 with $\mathcal{Y}_{c} \in \operatorname{PMod}_{2}(\mathscr{T})$. Let $\phi: \mathcal{Y}_{c} \rightarrow Y_{c}^{+}$be an elementary modification of type I, contracting a curve $C$, such that $C$ does not meet the singular locus of the special component $\left(\mathcal{Y}_{c}\right)_{\omega}$. Then there is a flopping contraction $\mathcal{Y} \rightarrow \mathcal{Z}$, such that for the induced flop $\mathcal{Y}^{+}$, we have $\mathcal{Y}_{c}^{+}=Y_{c}^{+}$.

Proof. Let $Y_{\omega}$ be the surface obtained by applying $\phi$ to $\left(\mathcal{Y}_{c}\right)_{\omega}$. Let $D_{1} \cup D_{3}$ be the preimage of Sing $Y_{\omega}$ under the normalisation $Y_{\omega}^{v} \rightarrow Y_{\omega}$. As $C$ does not meet the singular locus of the special component, $D_{1}^{2}=D_{3}^{2}=-1$. Hence $Y_{c}^{+} \in \operatorname{PMod}_{2}(\mathscr{T})$ by Proposition 5.1.

Corollary 6.8. Let $\mathcal{Y}$ be a model of the DNV family of degree 2, with $\mathcal{Y}_{c} \in \operatorname{PMod}_{2}$. Let $\phi: \mathcal{Y}_{c} \rightarrow Y_{c}^{+}$be an elementary modification of type II, contracting a curve $C$. Then there is a flopping contraction $\mathcal{Y} \rightarrow \mathcal{Z}$, such that for the flop $\mathcal{Y}^{+}$we have $\mathcal{Y}_{c}^{+}=Y_{c}^{+}$.

Proof. If $\mathcal{Y}$ is of class $\mathscr{P}$ and we apply a type II modification, then $Y_{c}^{+}$has dual intersection graph $\mathscr{T}$, as follows from the description of elementary modifications in Section 1.1. By the definition of elementary modifications of type II, $Y_{c}^{+}$fullfills the condition of Proposition 5.1 and hence $Y_{c}^{+}$is projective. The result then follows from Proposition 6.6.

If $\mathcal{Y}$ is of class $\mathscr{T}$, then $Y_{c}^{+}$has dual intersection graph $\mathscr{P}$. By the definiton of elementary modifications of type II, $C$ is the smooth component of the restriction of the double locus of $\mathcal{Y}_{c}$ to $\left(\mathcal{Y}_{c}\right)_{\omega}$. Let $A$ be an ample line bundle on $\left(\mathcal{Y}_{c}\right)_{\omega}^{v}$ and let $C_{1}, C_{2}$ be the components of the preimage of $C$ under $\left(\mathcal{Y}_{c}\right)_{\omega}^{v} \rightarrow\left(\mathcal{Y}_{c}\right)_{\omega}$. Here, we choose $A$ such that $A \cdot C_{1}=A \cdot C_{2}$ which can be done as $\left(\mathcal{Y}_{c}\right)_{\omega}$ is projective. Note that $C_{i}^{2}=-1$. So $L^{\prime}=A+\left(A \cdot C_{i}\right) C_{i}$ has degree 0 on the $C_{i}$ and strictly positive degree on all other curves. By the usual arguments, there is a divisor $L$ on $\mathcal{Y}_{c}$ that restricts to a positive multiple of $L^{\prime}$ on $\left(\mathcal{Y}_{c}\right)_{\omega}$ and to an ample divisor on the remaining components. It follows that there is a nef divisor $\mathcal{L}$ on $\mathcal{Y}$ that has degree zero 
precisely on $C$. The birational morphism induced by $\mathcal{L}$ is a flopping contraction with flopping curve $C$. Hence there is a model $\mathcal{Y}^{+}$with properties as required.

Remark 6.9. We emphasize that in Corollaries 6.7 and 6.8, the exceptional locus is precisely the curve $C$.

If $C$ is a curve on an component $Y$ of the central fibre of a model $\mathcal{Y} \rightarrow S$, we say that $C-$ considered as a curve on $\mathcal{Y}-$ is a $(-1)$-curve if the components of the preimage $C^{v}$ under the normalisation $Y^{v} \rightarrow Y$ are $(-1)$-curves.

Definition 6.10. Let $f: \mathcal{Y} \rightarrow \mathcal{Z}$ be a flopping contraction with flopping curve $C$ an irreducible (-1)-curve. The flop $\mathcal{Y} \rightarrow \mathcal{Y}^{+}$defined by $f$ is a type I flop if $C$ is not contained in the double locus $D$ of the central fibre $\mathcal{Y}_{c}$ and a type II flop if $C \subset D$.

Note that type I and type II flops are simply global versions of type I and type II elementary modifications.

In the degree 2 case, we can give a refined version of Lemma 6.1 in case of flopping contractions on models with central fibre in $\mathrm{PMod}_{2}$.

Proposition 6.11. Let $\mathcal{Y} \rightarrow S$ be a model of the DNV family of degree 2 such that $\mathcal{Y}_{c} \in \operatorname{PMod}_{2}$. Let $\pi: \mathcal{Y} \rightarrow \mathcal{Z}$ be a flopping contraction, given be the contraction of an extremal ray $R$. Then the exceptional locus $\operatorname{Ex}(\pi)$ is an irreducible (-1)-curve.

Proof. The idea of the proof is the following: assume that $\pi: \mathcal{Y} \rightarrow \mathcal{Z}$ has reducible exceptional locus $\operatorname{Ex}(\pi)$. Then we shall show that one can find a contraction that contracts a proper subset of $\operatorname{Ex}(\pi)$. Hence the classes of the components of $\operatorname{Ex}(\pi)$ are not contained in an extremal ray $R$, giving a contradiction.

By Proposition 6.3 all connected components of the exceptional locus of a flopping contraction are $(-1)$-curves. If a component $C$ of $\operatorname{Ex}(\pi)$ is a $(-1)$-curve in the double locus, then there is a flopping contraction with exceptional locus $C$, by Corollary 6.8 .

So we can assume that all connected components $C_{i}$ of $\operatorname{Ex}(\pi)$ are given by interior $(-1)$-curves. As each component of the double locus Sing $\mathcal{Y}_{c}$ meets at most one $C_{i}$, there are at most 3 such components, $C_{1}, C_{2}, C_{3}$. Also, if $\mathcal{Y}_{c} \in \operatorname{PMod}_{2}(\mathscr{T})$, by Proposition 5.1, none of the $C_{i}$ meets the smooth component of the restriction of $\operatorname{Sing} \mathcal{Y}_{c}$ to $\left(\mathcal{Y}_{c}\right)_{\omega}$. Hence, by Corollary 6.7, we only need to show the Proposition for $\mathcal{Y}_{c} \in \operatorname{PMod}_{2}(\mathscr{P})$.

We show that in this case, $\operatorname{Ex}(\pi)$ is irreducible. Suppose there are two components $C_{1}, C_{2}$ in $\operatorname{Ex}(\pi)$. Write $\mathcal{Y}_{c}=Y_{1} \cup Y_{2} \cup Y_{3}$ and assume $C_{1} \subset Y_{1}$. Let $\xi_{i}=\sum_{j=1}^{3} D_{i j}-D_{j i}$. This defines a divisor in $\operatorname{Pic}(\mathcal{Y} / S)$, by Lemma 2.10 and maximality of the model $\mathcal{Y} \rightarrow S$. Then $\xi_{1} \cdot C_{1}=1$ and $\xi_{1} \cdot C_{2}=-1$ if $C_{2}$ is not contained in $Y_{1}$. If $C_{2}$ is contained in $Y_{1}$, then $C_{2} \cdot D_{1 k}=1$ for some $k \in\{2,3\}$. Then $\xi_{k} \cdot C_{1}=0$ and $\xi_{k} \cdot C_{2}=-1$. Hence $C_{1}$ is not numerically equivalent to a positive multiple of $C_{2}$. This is a contradiction to $\pi=\operatorname{contr}_{R}$ and we can conclude that $\operatorname{Ex}(\pi)=C_{1}$.

For later reference, we record the following immediate corollary.

Corollary 6.12. Let $\mathcal{Y} \rightarrow S$ be a model of the DNV family of degree 2. Let $\pi: \mathcal{Y} \rightarrow \mathcal{Z}$ be a flopping contraction. Then the flop $\mathcal{Y} \rightarrow \mathcal{Y}^{+}$defined by $\pi$ is a type I flop or a type II flop.

Remark 6.13. We can make Remark 6.4 more precise: if $\phi: \mathcal{Y} \rightarrow \mathcal{Y}^{+}$is a flop, then the dual intersection complexes of $\mathcal{Y}$ and $\mathcal{Y}^{\prime}$ are the same if $\phi$ is of type I and they are distinct if $\phi$ is of type II.

Lemma 6.14. Let $\mathcal{Y}$ be a model of the DNV family of degree 2 with $\mathcal{Y}_{c} \in \operatorname{PMod}_{2}(\mathscr{P})$. Assume that we have a birational map $\phi: \mathcal{Y} \rightarrow \mathcal{Y}^{\prime}$ to another model $\mathcal{Y}^{\prime}$. Let $F^{\prime}$ be an ample divisor on $\mathcal{Y}^{\prime}$ with birational transform $F$ on $\mathcal{Y}$. Consider a factorization of $\phi$ into $F$-flops:

$$
\mathcal{Y} \rightarrow \mathcal{Y}_{1} \rightarrow \cdots \rightarrow \mathcal{Y}_{i} \stackrel{\phi_{i}}{\rightarrow} \mathcal{Y}_{i+1} \rightarrow \cdots \rightarrow \mathcal{Y}_{n} \stackrel{\sim}{\rightarrow} \mathcal{Y}^{\prime}
$$

Then at most one $\phi_{i}$ is of type II. In particular, if both $\mathcal{Y}$ and $\mathcal{Y}^{\prime}$ are of class $\mathscr{P}$, all $\phi_{i}$ are type I flops. 
Proof. Write $\mathcal{Y}_{c}=Y_{1} \cup Y_{2} \cup Y_{3}$. Without loss of generality we can assume the first flop in the factorisation to be of type II with exceptional locus $Y_{1} \cap Y_{2}$. Let $Y=Y_{3}$. Let $Y^{\prime}$ be the component of $\mathcal{Y}_{c}^{\prime}$ which is the transform of $Y$. Then $Y^{\prime}$ is a surface such that the preimage of the restriction of Sing $\mathcal{Y}_{c}^{\prime}$ to $Y^{\prime}$ under the normalisation morphism has 4 components, 2 of which, say $D_{1}, D_{2}$, are (-1)-curves that are identified under the normalisation map, giving a smooth component $D_{s}$. The images of the curves in $\Gamma_{Y}$ together with the interior (-1)-curves meeting $D_{1}$ and $D_{2}$ define a system of curves that is a basis of $\operatorname{Pic}\left(\left(Y^{\prime}\right)^{v}\right)$, and as in Proposition 5.1, one shows that for all $\phi_{i}, i \geq 2, \operatorname{Ex}\left(\phi_{i}\right)$ cannot meet $D_{s}$, where we use the notation as in Remark 6.5. Hence $F_{k} \cdot\left(D_{s}\right)_{k}>0$ for all $k \geq 2$. Thus any flopping curve in the sequence is an interior $(-1)$-curve, so all other flops are of type I.

\subsection{Birational Automorphisms}

We first fix some language. See Remark 6.5 for notation.

Definition 6.15. Let $\mathcal{Y}$ be model of the DNV family of degree 2 with $\mathcal{Y}_{c} \in \operatorname{PMod}_{2}(\mathscr{P})$. Let $F \in \operatorname{Mov}(\mathcal{Y} / S)$. Let $C$ be a curve with $F \cdot C<0$ that generates an extremal ray and let $\phi_{1}: \mathcal{Y} \rightarrow \mathcal{Y}_{1}$ be the flop given by contracting $C$. Further, let $\phi^{\prime}=\prod_{i=2}^{n} \phi_{i}: \mathcal{Y}_{1} \rightarrow \mathcal{Y}^{\prime}$ be a sequence of $\left(\phi_{1}\right)_{*} F$-flops and assume all flops $\phi_{i}$ in the sequence $\phi^{\prime}$ are of type I. Set $\phi=\phi^{\prime} \circ \phi_{1}$.

(i) Let $C^{+}$be the flopped curve of $\phi_{1}$. Any curve $C^{\prime}$ that is the birational transform of $C^{+}$under a subsequence of flops from $\phi^{\prime}$ is called a $\phi$-flop of $C$.

(ii) Let $C^{\prime}$ be a $\phi$-flop of $C$ that is itself a flopping curve. Any curve birational to the flopped curve $\left(C^{\prime}\right)^{+}$ under a map $\prod_{i=k}^{m} \phi_{i}, k, m \leq n$, is also called a $\phi$-flop of $C$.

Remark 6.16. If $\phi$ is the sequence

$$
\mathcal{Y} \rightarrow \mathcal{Y}_{1} \rightarrow \cdots \rightarrow \mathcal{Y}_{i} \stackrel{\phi_{i}}{\rightarrow} \mathcal{Y}_{i+1} \rightarrow \cdots \rightarrow \mathcal{Y}_{n} \stackrel{\sim}{\rightarrow} \mathcal{Y}^{\prime}
$$

and $E$ is a curve on some $\mathcal{Y}_{k}$ in the factorisation, we have a sequence $\phi^{\prime}$ of flops defined by the tail

$$
\mathcal{Y}_{k} \rightarrow \mathcal{Y}_{k+1} \rightarrow \cdots \rightarrow \mathcal{Y}_{i} \stackrel{\phi_{i}}{\rightarrow} \mathcal{Y}_{i+1} \rightarrow \cdots \rightarrow \mathcal{Y}_{n} \stackrel{\sim}{\rightarrow} \mathcal{Y}^{\prime}
$$

In this situation, if $E^{\prime}$ is a $\phi^{\prime}$-flop of $E$ we will also call $E^{\prime}$ a $\phi$-flop of $E$.

Definition 6.17. Let $\mathcal{Y} \rightarrow S$ be a model of the DNV family in degree 2 with $\mathcal{Y}_{c} \in \operatorname{PMod}_{2}(\mathscr{P})$. Let $Y \subset \mathcal{Y}_{c}$ be a component of the central fibre and let $D_{1}$ be a component of the anticanonical cycle $D=D_{1}+D_{2}$ of $Y$. Let $C$ be an interior (-1)-curve on $Y$ meeting $D_{1}$

(i) $C$ is called alone if there is no $H$ in $\Gamma_{Y}$ with $H \cdot D_{1}=H \cdot D_{2}=1$ and also

$$
\left\{E \in \Gamma_{Y} \mid E \cdot D_{1}=1 \text { and } E^{2}=-1\right\}=\{C\} .
$$

(ii) $C$ is called an annex if $C$ is not alone and either meets a unique $v \in \Gamma_{Y}$ or is the unique interior $(-1)$-curve of $Y$ meeting $D_{1}$.

(iii) If $C$ is not alone, the curve $C^{\prime} \in \Gamma_{Y} \backslash C$ meeting $D_{1}$ is the companion of $C$.

Some illustrations of this definition can be found in Figure 18.

Definition 6.18. Let $\phi$ be a sequence of $F$-flops

$$
\mathcal{Y} \rightarrow \mathcal{Y}_{1} \rightarrow \cdots \rightarrow \mathcal{Y}_{i} \stackrel{\phi_{i}}{\rightarrow} \mathcal{Y}_{i+1} \rightarrow \cdots \rightarrow \mathcal{Y}_{n} \stackrel{\sim}{\rightarrow} \mathcal{Y}^{\prime}
$$

and $F$ be a divisor on $\mathcal{Y}$. Assume all flops $\phi_{i}$ are of type I. Let $I=\{0, \ldots, n\}$ be the index set, where we assign the index 0 to $\mathcal{Y}$. Let $E$ be a curve on some $\mathcal{Y}_{k}$ that is the flopped curve of $\phi_{k}$.

(i) An E-sequence is a collection of indices $K_{E}^{\phi} \subset I$ such that for all $k \in K_{E}^{\phi}$, the flopping curve of $\phi_{k}: \mathcal{Y}_{k} \rightarrow \mathcal{Y}_{k+1}$ is either $E$ or a $\phi$-flop of $E$. 

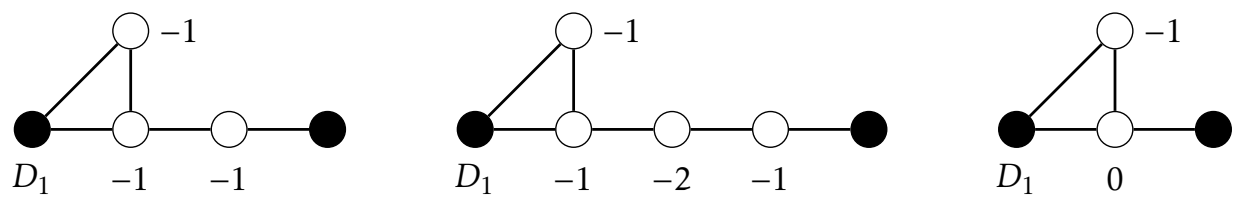

Figure 18. Some examples of (augmented) curve structures where none of the (-1)-curves meeting $D_{1}$ is alone. In all examples, the $(-1)$ curve $v$ meeting $D_{1}$ and exactly one curve in $\Gamma_{Y} \backslash\{v\}$ is an annex. Also, both curves meeting $D_{1}$ are companions (of each other).

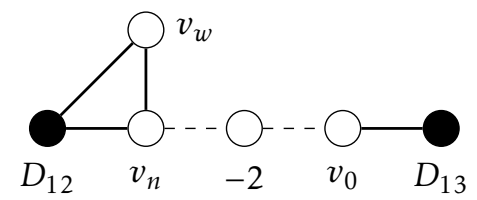

Figure 19. The curve structure in Example 6.20

(ii) Set $m_{1}\left(K_{E}^{\phi}\right)=\min _{K_{E}^{\phi}} k$ and $m_{2}\left(K_{E}^{\phi}\right)=\max _{K_{E}^{\phi}} k$. An $E$-sequence $K_{E}^{\phi}$ is complete if for any $i$ such that the flopping curve of $\phi_{i}$ is $E$ or a $\phi$-flop of $E, i \notin K_{E}^{\phi}$ implies $i \notin\left[m_{1}\left(K_{E}^{\phi}\right), m_{2}\left(K_{E}^{\phi}\right)\right]$.

(iii) For any $k \in K_{E}^{\phi}$, let $E(k)$ denote the flopped curve of $\phi_{k}$. A complete $E$-sequence $K_{E}^{\phi}$ is directed if either $\left(D_{i j}\right)_{k} \cdot E(k)=1$ implies $i<j$ for all $k \in K_{E}^{\phi}$ or $\left(D_{i j}\right)_{k} \cdot E(k)=1$ implies $i>j$ for all $k \in K_{E}^{\phi}$.

(iv) A directed $E$-sequence $K_{E}^{\phi}$ is called initial if for any $i$ such that the flopping curve of $\phi_{i}$ is $E$ or a $\phi$-flop of $E, i \notin K_{E}^{\phi}$ implies $i>m_{2}\left(K_{E}^{\phi}\right)$.

(v) An initial $E$-sequence $K_{E}^{\phi}$ is called exhaustive if for any $i$ such that the flopping curve of $\phi_{i}$ is $E$ or a $\phi$-flop of $E, i \in K_{E}^{\phi}$.

Definition 6.19. Let $\mathcal{Y}$ be a model of the DNV family of degree 2 with $\mathcal{Y}_{c} \in \operatorname{PMod}_{2}(\mathscr{P})$. Let us denote $\mathcal{Y}_{c}=Y_{1} \cup Y_{2} \cup Y_{3}$ the central fibre. Let $F \in \operatorname{Mov}(\mathcal{Y})$. Let $C$ be an interior $(-1)$-curve on $Y_{1}$ with $F \cdot C<0$ that generates an extremal ray and let $\phi_{1}: \mathcal{Y} \rightarrow \mathcal{Y}_{1}$ be the flop given by contracting $C$. Assume the numbering is such that $D=D_{12}$ is the component of the anticanonical divisor met by $C$. Let $C^{+}$be the flopped curve of $\phi_{1} . C$ is good for $D$ or $D$-good if for any sequence of $F$-flops $\phi=\phi^{\prime} \circ \phi_{1}$ as in Definition 6.15, the following holds:

(i) if $C^{\prime}$ is the flopped curve of $\mathcal{Y}_{k} \rightarrow \mathcal{Y}_{k+1}$ and if there is an initial $C$-sequence $K_{C}^{\phi}$ with $k \in K_{C}^{\phi}$, then $C^{\prime}$ is alone.

(ii) Let $E$ be the curve in $\Gamma_{\left(Y_{2}\right)_{1}}$ with $E^{2}<0$ and $E \cdot C^{+}=1$. Suppose there is a birational transform $E_{r}$ of $E$ that is the flopping curve of some $\mathcal{Y}_{r} \rightarrow \mathcal{Y}_{r+1}$. If $E^{\prime}$ is the flopped curve of $\mathcal{Y}_{k} \rightarrow \mathcal{Y}_{k+1}$ and there is an initial $E_{r}$-sequence $K_{E}^{\phi}$, with $k \in K_{E_{r}}^{\phi}$, then $E^{\prime}$ is alone.

The following example is a model for our future considerations.

Example 6.20. We give an example of $D$-good curves and an exhaustive sequence. Let $\mathcal{Y}$ be a model with central fibre $\mathcal{Y}_{c} \in \operatorname{PMod}_{2}(\mathscr{P})$. Write $\mathcal{Y}_{c}=Y_{1} \cup Y_{2} \cup Y_{3}$ and suppose that $\Gamma_{Y_{1}}$ is tamely degenerate and has an exceptional vertex $v_{0}$. In particular, $v_{0}^{2}=-1$. Assume moreover that for the unique vertex $v_{w} \in \Gamma_{Y_{1}} \backslash\left\{L\left(v_{0}\right)\right\}$, $v_{w}^{2}=-1$. Let $v_{n}$ be the vertex on which $L\left(v_{0}\right)$ ends. We have $v_{n}^{2}=-1$. Let $D_{12}$ be the component of the anticanonical divisor met by $v_{w}$, see Figure 19 .

We show that $C_{v_{w}}$ is $D_{12}$-good. Note that $D_{12}^{2}=3$. Let $\phi$ be a sequence of $F$-flops, such that $\mathcal{Y} \rightarrow \mathcal{Y}_{1}$ has flopping curve $C:=C_{v_{w}}$ and assume all flops $\phi_{i}$ in $\phi$ to be of type I. Let $E$ be the unique curve of $\Gamma_{\left(Y_{2}\right)_{1}}$ met by $C^{+}$. We have $\left(C_{v_{n}}\right)_{1}^{2}=0$. Assume no birational transform $E_{r}$ of $E$ is a flopping curve of $\phi_{r}$. Then no birational transform of $\left(C_{v_{w}}\right)$ meets a birational transform of $D_{23}$. We first assume that there exists some 
$p \geq 2$ such that $\operatorname{Ex}\left(\phi_{p}\right)$ meets $\left(D_{12}\right)_{p}$ or $\left(D_{21}\right)_{p}$. Choose the minimal such $p$. It then follows by minimality that $\operatorname{Ex}\left(\phi_{p}\right) \cdot\left(D_{12}\right)_{p}=1$. But then $\operatorname{Ex}\left(\phi_{p}\right)=\left(C_{v_{n}}\right)_{p}$, which is impossible as $\left(C_{v_{n}}\right)_{1}^{2} \geq 0$. Therefore $C_{v_{w}}$ is $D_{12 \text {-good. }}$

Now, assume $E_{t}$ is the flopping curve of $\phi_{t}$, where $t$ is chosen minimal. Necessarily, $\operatorname{Ex}\left(\phi_{t}\right) \cdot\left(D_{23}\right)_{t}=1$. Let $E^{+}$be the flopped curve of $\phi_{t}$. It is straightforward to calculate $\left(D_{32}\right)_{t+1}^{2}=-12$ : $\left(Y_{2}\right)_{t}$ is obtained from $Y_{\mathscr{P}}$ by blowing up one interior special point 4 times and blowing down 10 times in the other interior special point. Flopping $E_{t}$ corresponds to a further blow down, giving $\left(D_{32}\right)_{t+1}^{2}=-12$. So $E^{+}$is the only curve in $\Gamma_{\left(Y_{3}\right)_{t+1}}$ meeting $\left(D_{32}\right)_{t+1}$. In particular, $E^{+}$is alone. No birational transform of $E^{+}$can be a flopping curve: such a curve $E_{s}^{+}$would be $F_{s}$-negative, implying that there is a minimal index $k$ such that $E_{k}^{+}$meets $\left(D_{23}\right)_{k}$. But this implies that all curve structures of the components of the central fibre of $\mathcal{Y}_{k}$ are degenerate, a contradiction to Proposition 5.2. Hence $C$ is $D_{12}$-good and there is an exhaustive $C$-sequence.

Similar arguments show that $C_{v_{n}}$ is $D_{12}$-good.

In order to make the statements of the following lemmas lighter, we introduce the following setup.

Setting 6.21. Let $\mathcal{Y}$ and $\mathcal{Y}^{\prime}$ be models of the DNV family of degree 2, with $\mathcal{Y}_{c}, \mathcal{Y}_{c}^{\prime} \in \operatorname{PMod}_{2}(\mathscr{P})$. Write $\mathcal{Y}_{c}=Y_{1} \cup Y_{2} \cup Y_{3}$. Let $\phi: \mathcal{Y} \rightarrow \mathcal{Y}^{\prime}$ be a birational map and let $C$ be an interior (-1)-curve on the component $Y_{1}$ meeting $Y_{1} \cap Y_{2}$. Let $F^{\prime}$ be ample on $\mathcal{Y}^{\prime}$ and denote its pullback under $\phi$ by $F$. Let

$$
\mathcal{Y} \rightarrow \mathcal{Y}_{1} \rightarrow \cdots \rightarrow \mathcal{Y}_{i} \stackrel{\phi_{i}}{\rightarrow} \mathcal{Y}_{i+1} \rightarrow \cdots \rightarrow \mathcal{Y}_{n} \stackrel{\sim}{\rightarrow} \mathcal{Y}^{\prime}
$$

be a factorisation into $F$-flops.

We will assume this setting in Lemmas 6.22, 6.25, 6.26, 6.28, 6.30, 6.31, 6.35 and in Corollary 6.27.

Lemma 6.22. Assume Setting 6.21. Suppose that the curve $C$ is not an annex and $F \cdot C \geq 0$. If there is a $p$ such that $\operatorname{Ex}\left(\phi_{p}\right)=C_{p}$ and $C_{p} \cdot\left(D_{12}\right)_{p}=1$, then there exists $q<p$ with $C_{q} \cdot\left(D_{13}\right)_{q}=1$.

Proof. Suppose there is a $p$ such that $\operatorname{Ex} \phi_{p}=C_{p}$ but $C_{q} \cdot\left(D_{13}\right)_{q}=0$ for all $q \leq p$. We have $F_{p} \cdot C_{p}<0$, hence there must be some minimal $k<p$ such that $C_{k}$ meets the flopping curve $H$ or the flopped curve $H^{+}$of $\phi_{k}$. As $C_{q} \cdot\left(D_{13}\right)_{q}=0$ for all $q<p$, in the first case, $H \cdot\left(D_{12}\right)_{k}=1$ and in the second case, $H^{+} \cdot\left(D_{12}\right)_{k+1}=1$. The first case can only occur if $C$ is not alone and $H=C_{k}^{\prime}, C^{\prime}$ the annex meeting $C$. So in this case, $C_{k+1}^{2}=0$. The curve $H$ is $D_{12}$-good by the argument in Example 6.20. There, it is also shown that there is an exhaustive $H$-sequence, so it follows that $C_{s}^{2} \geq 0$ for $s \geq k+1$. Thus, $C_{s}$ is not contracted for any $s \geq k+1$, a contradiction to $\operatorname{Ex} \phi_{p}=C_{p}$. Consider the second case. We have $F \cdot H^{+}>0$ and by our assumptions, there is an $h$ such that $\operatorname{Ex}\left(\phi_{h}\right)=H_{h}^{+}$and then arguing as before, there is $j<h$ such that $H_{k}^{+}$meets the flopping curve $G$ or the flopped curve $(G)^{+}$of $\phi_{j}$. As $H^{+}$is not an annex, as above, we conclude then that either $\left(H_{i}^{+}\right)^{2}=0$ for $i>k$ or $G^{+} \cdot H_{k}^{+}=1$ and $H_{k}^{+}$is disjoint from the boundary. As there are only finitely many flops in the sequence, this procedure stops with a curve $H^{\prime}$ such that either there is an $l<p$ such that $F_{i} \cdot H_{i}^{\prime}>0$ and $H_{i}^{\prime} \cdot\left(D_{12}\right)_{i}=1$ for $i>l$ or $\left(H_{i}^{\prime}\right)^{2}=0$ and $H_{i}^{\prime} \cdot\left(D_{12}\right)_{i}=1$ for $i>l$, a contradiction.

Definition 6.23. Assume Setting 6.21.

(i) Assume $\mathcal{Y} \rightarrow \mathcal{Y}_{1}$ has flopping curve $C$. Let $C^{+} \subset\left(Y_{2}\right)_{1}$ be the flopped curve and assume $C^{+}$is not alone. Let $E$ be the $(-1)$-curve meeting $\left(D_{21}\right)_{1}=\left(Y_{2}\right)_{1} \cap\left(Y_{1}\right)_{1}$ with $E \cdot C^{+}=1$. Set $D_{R}:=D_{21}$. Assume there is an index $q$ such that $E_{q}$ meets $\left(D_{R}\right)_{q}$ and is the flopping curve of $\mathcal{Y}_{q} \rightarrow \mathcal{Y}_{q+1}$. This $q$ is unique as $E_{q}$ is $\left(D_{R}\right)_{q}$-good. In this case we say that $C$ is replaced by $E$ and say $E$ is the replacement of $C$.

(ii) Suppose $F \cdot C \geq 0$. Assume $V:=\left\{i \in \mathbb{N} \mid C_{i} \cdot\left(D_{13}\right)_{i}=1\right\} \neq \varnothing$ and let $p$ be its minimal element. Let $E$ be the flopping curve of $\phi_{p-1}$. Also, suppose that if $s$ is the minimal integer $s>p$ such that $C_{s}$ is the flopped curve of $\phi_{s}$, then $s \notin V$. If there is such an $s$, we say that $C$ is reflected. If $C$ is reflected, there is a minimal $q, s>q>p$ such that $q \notin V$. We set $D_{R}:=D_{23}$. The flopped curve $C^{R}$ of $\phi_{q}$ is the reflection of $C$. 
The curve $D_{R}$ is the $R$-locus of the replacement or the reflection. The index $q$ such that the flopped curve of $\phi_{q}$ is the replacement or the reflection of $C$ is called the index of the replacement resp. the reflection.

Remark 6.24. If a $\phi$-flop $C^{\prime}$ of $C$ is replaced or reflected we will also call the replacement $E$ of $C^{\prime}$ a replacement or a reflection of $C$.

Lemma 6.25. Assume Setting 6.21. Further assume that $C$ is alone and $F \cdot C \geq 0$. Suppose $C$ is reflected, with reflection $C^{R}$. Suppose $p$ is the minimal element of $\left\{i \in \mathbb{N} \mid C_{i} \cdot\left(D_{13}\right)_{i}=1\right\}$, which exists by definition. Let $E^{+}$be the flopped curve of $\phi_{p-1}$. Let $q$ be the index of the reflection. If $E^{+}$is not an annex, then $E^{+}$is not alone and $\operatorname{Ex} \phi_{q}$ is a birational transform of the annex $E^{a}$ meeting $E^{+}$.

Proof. By definition of reflection, there is a $b$ with $C_{b} \cdot\left(D_{12}\right)_{b}=1$ and $C_{b}$ the flopping curve of $\phi_{b}$. There is a $p, p<b$ with $C_{p} \cdot\left(D_{13}\right)_{p}=1$. Assume $p$ minimal with this property. As in the statement, let $E^{+} \subset\left(Y_{3}\right)_{p}$ be the flopped curve of $\phi_{p-1}$. By assumption, $E^{+}$is not an annex. Note that if $C_{p}$ is not alone, it is necessarily not an annex. Also, the annex $C_{p}^{a}$ is $D_{13}$-good and hence is not a flopped curve for $i \geq p$, as $C$ is reflected.

If $E^{+}$is not alone, let $E^{a}$ be the annex and suppose $C^{R}$ is not a $\phi$-flop of $E^{a}$. In any event $\left(E^{+}\right.$alone or not) as $C$ is reflected, there is a $b^{\prime}, b^{\prime}<b$ with $E_{b^{\prime}}^{+}$the flopping curve of $\phi_{b^{\prime}}$ and $E_{b^{\prime}}^{+} \cdot\left(D_{31}\right)_{b^{\prime}}=1$. By Lemma 6.22 , there is $p^{\prime}, p^{\prime}<b^{\prime}$, such that $E_{p^{\prime}}^{+} \cdot\left(D_{32}\right)_{p^{\prime}}=1$, which we again assume minimal.

Let $G^{+} \subset\left(Y_{2}\right)_{p^{\prime}}$ be the flopped curve of $\phi_{p^{\prime}-1}$. Suppose $G^{+}$is alone. Again, it follows that there is $b^{\prime \prime}$ with $p^{\prime}<b^{\prime \prime}<b^{\prime}$ such that for the flopping curve $G_{b^{\prime \prime}}^{+}$of $\phi_{b^{\prime \prime}}$ one has $G_{b^{\prime \prime}}^{+} \cdot\left(D_{23}\right)_{b^{\prime \prime}}=1$. By Lemma 6.22, there is $p^{\prime \prime}, p^{\prime}<p^{\prime \prime}<b^{\prime \prime}$ such that $G_{p^{\prime \prime}}^{+} \cdot\left(D_{21}\right)_{p^{\prime \prime}}=1$, which we again assume minimal. So $\Gamma_{\left(Y_{2}\right)_{p^{\prime \prime}}}$ and $\Gamma_{\left(Y_{3}\right)_{p^{\prime \prime}}}$ are degenerate. If $G^{+}$is not alone, it follows that $\Gamma_{\left(Y_{2}\right)_{p^{\prime}}}$ and $\Gamma_{\left(Y_{3}\right)_{p^{\prime}}}$ are degenerate.

In both cases, by projectivity, it follows from Proposition 5.2 that $\Gamma_{\left(Y_{1}\right)_{j}}$ for $j \in\left\{p^{\prime}, p^{\prime \prime}\right\}$ is non-degenerate. By the assumption that $C$ is reflected, we have $C_{s} \cdot\left(D_{12}\right)_{s}=1$ for the minimal $s>p$ such that $C_{s}$ is the flopped curve of $\phi_{s}$. Hence it follows from Lemma 6.22 (applied to the exceptional vertex of $\Gamma_{\left(Y_{1}\right)_{j}}$ distinct from $C$ ) that $\left(G^{+}\right)_{i} \cdot\left(D_{21}\right)_{i}=1$ for all $i \geq j$. But then $C$ is not reflected, a contradiction. Hence we conclude that $E^{+}$is not alone and $C^{R}$ is a $\phi$-flop of $E^{a}$. Now, it follows by the argument in Example 6.20 that $\operatorname{Ex}\left(\phi_{q}\right)$ is indeed a birational transform of $E^{a}$.

Lemma 6.26. Assume Setting 6.21. Suppose $C$ is the flopping curve of $\phi_{0}$. If $C$ is D-good, then there is an exhaustive $C$-sequence $K_{C}^{\phi}$.

Proof. Let $K_{C}^{\phi}$ be a maximal initial $C$-sequence. To get a contradiction, suppose $K_{C}^{\phi}$ is not exhaustive. We can assume that $K_{C}^{\phi}=\{0\}$. Let $C^{+}$be the flopped curve of $\phi_{0}$. Being a $\phi$-flop of $C, C^{+}$is alone and $F_{1} \cdot C^{+}>0$. As $K_{C}^{\phi}$ is not exhaustive, by definition, $C^{+}$is reflected.

So by assumption, there is some $b$ with $C_{b}^{+} \cdot\left(D_{12}\right)_{b}=1$ and $C_{b}$ the flopping curve of $\phi_{b}$. There is a $p$, $p<b$ with $C_{p} \cdot\left(D_{13}\right)_{p}=1$. Assume $p$ minimal with this property. Let $E^{+} \subset\left(Y_{3}\right)_{p}$ be the flopped curve of $\phi_{p-1}$. As $C$ is $D_{12}$ good, $E^{+}$is alone. In particular, $E^{+}$is not an annex. Being alone, $E^{+}$does not have a companion. From Lemma 6.25 we conclude that $C^{+}$is not reflected and hence $K_{C}^{\phi}$ is exhaustive.

Corollary 6.27. Suppose $F \cdot C \geq 0$ and assume that there is an integer $p$ such that $\operatorname{Ex} \phi_{p}=C_{p}$ and $C_{p} \cdot\left(D_{12}\right)_{p}=1$. Then $C$ is alone.

Proof. Suppose $C$ is not alone. As $F \cdot C \geq 0$, there must be some minimal $k, k<p$, such that $C_{k}$ meets the flopping curve $H$ or the flopped curve $H^{+}$of $\phi_{k}$. Suppose first that $C$ is an annex. Let $C^{\prime}$ be the companion of $C$. Suppose we are in the first case, i.e. $C_{k}$ meets the flopping curve $H$. Then $H=C_{k}^{\prime}$ as $C_{k} \cdot D_{12}=1$. By the argument in Example 6.20, $C^{\prime}$ is $D_{12}$-good, and hence from Lemma 6.26, there is an exhaustive $C^{\prime}$-sequence, and hence $C_{i}^{2} \geq 0$ for all $i>k$ contradicting our assumptions, as in particular $C_{p}^{2}<0$.

Hence we are in the second case, i.e. $C_{k}$ meets the flopped curve $H^{+}$of $\phi_{k}$. Then $F_{k+1} \cdot H^{+}>0, H^{+}$ is not an annex and there is an $h$ such that $\operatorname{Ex}\left(\phi_{h}\right)=H_{h}^{+}$, with $H_{h}^{+} \cdot\left(D_{12}\right)_{h}=1$. By Lemma 6.22 , we have 
a minimal $l, l<h$ with $H_{l}^{+} \cdot\left(D_{13}\right)_{l}=1$. Note that the flopping curve of $\phi_{l-1}$ is $C_{l-1}^{\prime}$ which is necessarily $D_{13^{-}}$good and there is an exhaustive $C_{l-1}^{\prime}$-sequence $\{l-1, \ldots, v\}$, by Lemma 6.26 , implying that $C_{i}$ is disjoint from $\left(D_{12}\right)_{i}$ for $i>l$, a contradiction. Now suppose $C$ is not alone and not the annex. Let $E$ be the curve in $\Gamma_{Y_{1}}$ with $E \cdot C=1$ and $E \cdot D_{12}=0$. By a straightforward extension of the argument in the proof of Example 6.20 , one checks that $E$ and $C$ are $D_{13}$-good. By Lemma $6.22, C_{q} \cdot\left(D_{13}\right)_{q}=1$. Hence for some $r<q$, $E_{r}=\operatorname{Ex}\left(\phi_{r}\right)$ and $E_{r} \cdot\left(D_{13}\right)_{r}=1$. By Lemma 6.26, there is an exhaustive $E$-sequence $K_{E}^{\phi}$. As $C$ is $D_{13}$-good, our assumptions imply $K_{E}^{\phi}=\{r\}$. Then $C_{i} \cdot\left(D_{13}\right)_{i}=1$ for all $i>r$, a contradiction.

Lemma 6.28. Assume Setting 6.21. Suppose $F \cdot C \geq 0$ and assume $C$ is reflected, with reflection $C^{R}$. Let $E^{+}$ be the flopped curve of $\phi_{p-1}$, with $p$ the minimal element of $\left\{i \in \mathbb{N} \mid C_{i} \cdot\left(D_{13}\right)_{i}=1\right\}$. Let $q$ be the index of the reflection.

(i) If $E^{+}$is not an annex, then $E^{+}$is not alone and $\operatorname{Ex} \phi_{q}$ is a birational transform of the annex $E^{a}$ meeting $E^{+}$.

(ii) If $E^{+}$is an annex, then $\operatorname{Ex} \phi_{q}$ is a birational transform of the companion of $E^{+}$.

Proof. It follows from Corollary 6.27 that $C$ is alone. Hence item (i) is nothing but Lemma 6.25. If $E^{+}$is an annex, it follows from Corollary 6.27 that $\operatorname{Ex} \phi_{q}$ cannot be a birational transform of $E^{+}$. Hence it is necessarily a birational transform of the companion of $E^{+}$.

Remark 6.29. In particular, it follows that for the index $q$ of a replacement or reflection exactly one of $\Gamma_{\left(Y_{R}\right)_{q}}$ or $\Gamma_{\left(Y_{R}\right)_{q+1}}$ is a regular curve structure, where $Y_{R}$ is the component containing $D_{R}$.

Lemma 6.30. Assume Setting 6.21. Assume that $C$ is not alone, and let $W$ be the companion of $C$.

(i) Assume $\mathcal{Y} \rightarrow \mathcal{Y}_{1}$ has flopping curve $C$. Suppose $C$ and none of its $\phi$-flops is replaced or reflected. Then there is an exhaustive $C$-sequence $K_{C}^{\phi}$.

(ii) Suppose $C$ is not an annex. Suppose there is no index $i$ sucht that $\operatorname{Ex}\left(\phi_{i}\right) \cdot\left(D_{12}\right)_{i}=1$ and $\operatorname{Ex}\left(\phi_{i}\right)$ a $\phi$-flop of $C$ or $W$ or of a replacement of $C$. Then $D_{12}^{2} \geq\left(D_{12}\right)_{i}^{2}$ for all $i>0$.

Proof. The first part is immediate: take a maximal initial $C$-sequence $K_{C}^{\phi}=\{1, \ldots, p\}$. We can assume $K_{C}^{\phi}=\{1\}$. If the flopped curve $C^{+}$of $\phi_{p}$ is an annex, we are done by Corollary 6.27 , as in particular an annex is not alone. Else, we conclude from Lemma 6.22 and the assumption that there is no reflection.

For the second part, to obtain a contradiction, suppose the statement on intersection numbers is not true. Let $\mathcal{F}\left(D_{12}\right)$ be the collection of indices in $\{1, \ldots, n\}$ such that $i \in \mathcal{F}\left(D_{12}\right)$ implies $\operatorname{Ex}\left(\phi_{i}\right) \cdot\left(D_{12}\right)_{i}=1$ or $\operatorname{Ex}\left(\phi_{i}\right) \cdot\left(D_{21}\right)_{i}=1$. The assumption that the statement on intersection numbers is not true implies that there is a $p \in \mathcal{F}\left(D_{12}\right)$ with $\operatorname{Ex}\left(\phi_{p}\right) \cdot\left(D_{12}\right)_{p}=1$. Assume that $p$ is minimal with this property. Then there is $q \in \mathcal{F}\left(D_{12}\right)$ with $q<p$ such that $\operatorname{Ex}\left(\phi_{q}\right) \cdot\left(D_{21}\right)_{q}=1$, as else $\operatorname{Ex}\left(\phi_{p}\right)=C_{p}$ (or $\left.W_{p}\right)$, contrary to our assumptions. Suppose $q$ is chosen maximal. Let $H \subset\left(Y_{1}\right)_{q+1}$ be the flopped curve of $\phi_{q}$. In particular, $F_{q+1} \cdot H>0$. Also, $H$ is not an annex.

We have $\operatorname{Ex}\left(\phi_{p}\right)=H_{p}$, as the remaining possibility is that $H$ is not alone and $\operatorname{Ex}\left(\phi_{p}\right)=H_{p}^{a}$ with $H^{a}$ the annex meeting $H$. In that case, by the argument in Example $6.20, H_{p}^{a}$ is $D_{12}$-good, so $\left(H_{i}\right)^{2} \geq 0$ for all $i>p$ and $D_{12}^{2} \geq\left(D_{12}\right)_{i}^{2}$ for all $i>p$. By definition of $p$ we get $D_{12}^{2} \geq\left(D_{12}\right)_{i}^{2}$ for all $i>0$.

Hence, by item (i), $H$ is reflected. Thus, there is $r$ with $r<p$ such that $H_{r} \cdot\left(D_{13}\right)_{r}=1$. Also, by constrution, $H_{r} \cdot\left(D_{12}\right)_{r}=1$. Hence $\Gamma_{\left(Y_{3}\right)_{r}}$ and $\Gamma_{\left(Y_{1}\right)_{r}}$ are degenerate. So, by projectivity, it follows from Proposition 5.2 that $\Gamma_{\left(Y_{2}\right)_{r}}$ is non-degenerate. This implies that $\operatorname{Ex}\left(\phi_{q}\right)$ is $D_{21}$-good, so there is an exhaustive $\operatorname{Ex}\left(\phi_{q}\right)$-sequence by Lemma 6.26, contradicting $\operatorname{Ex}\left(\phi_{p}\right)=H_{p}$.

Lemma 6.31. Assume Setting 6.21. Further assume that $\mathcal{Y} \rightarrow \mathcal{Y}_{1}$ has flopping curve $C$.

(i) If $C$ is $D_{12}$-good, then $C$ and none of its $\phi$-flops is replaced or reflected.

(ii) If $C$ is an annex, then $\Gamma_{\left(Y_{1}\right)_{i}}$ is very degenerate for any $i>0$.

(iii) If $C$ is not alone and not an annex, then $\Gamma_{\left(Y_{1}\right)_{i}}$ is regular for any $i>0$. 
Proof. Item (i) is immediate: by definition of $D$-goodness, it follows that $C$ or its $\phi$-flops are not replaced. They are not reflected as there is an exhaustive $C$-sequence. For the remaining items, note that in both cases $C$ is $D_{12}$-good, implying the claim.

We are now able to prove a statement which is crucial for us (and which is specific to the degree 2 case).

Corollary 6.32. Let $\mathcal{Y} \rightarrow S$ be a model of the DNV family of degree 2 , of class $\mathscr{G}$ for $\mathscr{G} \in\{\mathscr{P}, \mathscr{T}\}$. Then there is a sequence of type I flops

$$
\mathcal{Y}_{\mathscr{G}} \rightarrow \cdots \rightarrow \mathcal{Y}
$$

Proof. We start with a birational map $\phi: \mathcal{Y}_{\mathscr{P}} \rightarrow \mathcal{Y}$ be a birational map. Let

$$
\mathcal{Y}_{\mathscr{P}} \rightarrow \mathcal{Y}_{1} \rightarrow \mathcal{Y}_{2} \rightarrow \cdots \rightarrow \mathcal{Y}_{i} \stackrel{\phi_{i}}{\rightarrow} \mathcal{Y}_{i+1} \rightarrow \cdots \rightarrow \mathcal{Y}_{n} \stackrel{\sim}{\rightarrow} \mathcal{Y}
$$

be a factorisation of $\phi$ into flops. By Proposition 6.3, each flop $\phi_{i}$ is either a type II flop or has exceptional locus given by disjoint interior (-1)-curves. Hence, if the set $\left\{i \in \mathbb{N} \mid \phi_{i}\right.$ is of type II $\}$ is empty, we are done. Otherwise it follows from Lemma 6.14 that this set consists of a single element $\{p\}$. Let $D_{i j}=Y_{i} \cap Y_{j}$ be a double curve with $\left(D_{i j}\right)_{p}$ contained in the exceptional locus of $\phi_{p}$. Let $F$ be the divisor defining the factorisation $\phi$, see Remark 6.5. If $C$ is an interior $(-1)$-curve on $Y_{i}$ meeting $D_{i j}$, then $C$ is $D_{i j}$-good by definition. It thus follows from Lemma 6.26 that $D_{i j}$ is disjoint from the flopped curve of $\phi_{i}$ for $i<p$. The same reasoning applies to $D_{j i}$. Hence $F \cdot D_{i j}=F \cdot D_{j i}<0$. So we can assume $p=0$. Then, all that remains to show is that the model $\mathcal{Y}_{1}$ obtained from $\mathcal{Y}_{\mathscr{P}}$ by a flop defined by a flopping contraction with exceptional locus a curve in the singular locus of $Y_{\mathscr{P}}$ can be obtained from $\mathcal{Y}_{\mathscr{T}}$ via type I flops. This is immediate: $\mathcal{Y}_{1}$ has two components that are weak del Pezzo surfaces of degree 3. For each such component, there is a tree of curves of length 2, i.e. a (-1)-curve and a (-2)-curve, meeting the interior special point. Flopping these trees gives the desired result.

We immediately obtain that the set of all models of the Dolgachev-Nikulin-Voisin family of degree 2 is indeed bijective to $\mathrm{PMod}_{2}$ :

Corollary 6.33. Let $\mathcal{Y} \rightarrow S$ be a model of the $D N V$ family of degree 2. Then $\mathcal{Y}_{c} \in \operatorname{PMod}_{2}$.

In particular, all models of the DNV family can be linked, using only type I flops, to one of the two maximal Kulikov models in $(-1)$-form. To go from maximal Kulikov models of type $\mathscr{P}$ to those of type $\mathscr{T}$, and vice versa, requires a type II flop.

Corollary 6.34. Let $\mathcal{Y} \rightarrow S$ and $\mathcal{Y}^{\prime} \rightarrow S$ be models of the DNV family of degree 2. Any birational $S$-map $\mathcal{Y} \rightarrow \mathcal{Y}^{\prime}$ factors into a sequence of type I and type II flops (and possibly an isomorphism).

Lemma 6.35. Assume Setting 6.21. Let $K_{C}^{\phi}$ be a maximal initial $C$-sequence that is not exhaustive. Let $p \notin K_{C}^{\phi}$ be the minimal index such that $C^{\prime}=\operatorname{Ex}\left(\phi_{p}\right)$ is a $\phi$-flop of $C$. Let $\phi^{\prime}$ be the composition

$$
\mathcal{Y}_{p} \rightarrow \mathcal{Y}_{1} \rightarrow \ldots \mathcal{Y}_{i} \stackrel{\phi_{i}}{\rightarrow} \mathcal{Y}_{i+1} \rightarrow \ldots \mathcal{Y}_{n}
$$

and let $K_{C^{\prime}}^{\phi^{\prime}}$ be a maximal initial $C^{\prime}$-sequence. If $\operatorname{Ex}\left(\phi_{i}\right) \cdot\left(D_{21}\right)_{i}=1$ for some $i \in K_{C^{\prime}}^{\phi^{\prime}}$, then $\left|K_{C}^{\phi}\right|=1$.

Proof. By assumption, $C$ is reflected. Let $q$ be the index, $D_{R}$ the $R$-locus of the reflection. Suppose the flopping curve of $\phi_{q}$ is not an annex. Then by the description in Lemma 6.28 there is a unique $r, r<q$, with $\operatorname{Ex}\left(\phi_{r}\right) \cdot D_{23}=1$ and no $i, i<q$ with with $\operatorname{Ex}\left(\phi_{i}\right) \cdot D_{32}=1$. Hence the curve structure $\Gamma_{Y_{2}}$ is either regular with $\left|\Gamma_{Y_{2}}\right|=2$ or very degenerate with $\left|\Gamma_{Y_{2}}\right|=1$ and $\Gamma_{Y_{3}}$ is very degenerate. Thus $\left|K_{C}^{\phi}\right|=1$.

By Lemma 6.28, the remaining possibility is that the flopping curve of $\phi_{q}$ is an annex. Suppose $D_{R} \subset Y_{R}$. Then $\Gamma_{\left(Y_{R}\right)_{q+1}}$ is very degenerate. Let $E \subset Y^{\prime}$ be the reflection of $C$. Let $D^{\prime}$ be the component of the double locus on $Y^{\prime}$ met by $E$. Then $E_{i} \cdot D_{i}^{\prime}=1$ for all $i>q$. Let $c$ be the maximal element of $K_{C}^{\phi}$ and let $C^{\prime}$ be the 
flopped curve of $\phi_{c}$. By definition $C_{q+1}^{\prime} \cdot E=1$. Also, if $C_{t}^{\prime}$ meets the boundary for some $t \geq c$, it is alone. It follows that $K_{C^{\prime}}^{\phi^{\prime}}$ is a singleton set, and as $\operatorname{Ex}\left(\phi_{i}\right) \cdot\left(D_{21}\right)_{i}=1$ for some $i \in K_{C^{\prime}}^{\phi^{\prime}}$, the same holds for $K_{C}^{\phi}$.

After these preliminaries, we can prove the main result of this section.

Proposition 6.36. Let $\mathcal{Y} \rightarrow S$ and $\mathcal{Y}^{\prime} \rightarrow S$ be models of the DNV family of degree 2, with $\mathcal{Y}_{c}, \mathcal{Y}_{c}^{\prime} \in \operatorname{PMod}_{2}(\mathscr{P})$. Let $\psi: \mathcal{Y} \rightarrow \mathcal{Y}^{\prime}$ and $\phi: \mathcal{Y} \rightarrow \mathcal{Y}^{\prime}$ be two birational maps. Write $\mathcal{Y}_{c}=\cup Y_{i}$ and $\mathcal{Y}_{c}^{\prime}=\cup Y_{i}^{\prime}$. Suppose

$$
\psi\left(Y_{i}\right) \subset Y_{j}^{\prime} \Leftrightarrow \phi\left(Y_{i}\right) \subset Y_{j}^{\prime}
$$

Let $A^{\prime}$ be an ample divisor on $\mathcal{Y}^{\prime}$. Let $A_{\phi}$ and $A_{\psi}$ be the birational transforms of $A^{\prime}$ under $\phi$ and $\psi$. Let $C$ be a $(-1)$-curve on $\mathcal{Y}$ that generates an extremal ray. Then

$$
A_{\phi} \cdot C<0 \Leftrightarrow A_{\psi} \cdot C<0 .
$$

Proof. Assume $A_{\phi} \cdot C<0$. Let

$$
\mathcal{Y} \rightarrow \mathcal{Y}_{1}^{\phi} \rightarrow \cdots \rightarrow \mathcal{Y}_{i}^{\phi} \stackrel{\phi_{i}}{\rightarrow} \mathcal{Y}_{i+1}^{\phi} \rightarrow \cdots \rightarrow \mathcal{Y}_{n}^{\phi} \stackrel{\sim}{\rightarrow} \mathcal{Y}^{\prime}
$$

and

$$
\mathcal{Y} \rightarrow \mathcal{Y}_{1}^{\psi} \rightarrow \cdots \rightarrow \mathcal{Y}_{i}^{\psi} \stackrel{\psi_{i}}{\rightarrow} \mathcal{Y}_{i+1}^{\psi} \rightarrow \cdots \rightarrow \mathcal{Y}_{m}^{\psi} \stackrel{\sim}{\rightarrow} \mathcal{Y}^{\prime}
$$

be factorisations of $\phi$ and $\psi$, such that $C$ is the flopping curve of $\mathcal{Y} \rightarrow \mathcal{Y}_{1}^{\phi}$. We show $A_{\psi} \cdot C<0$. By Proposition 6.11, $C$ is a (-1)-curve on a component, say $Y_{1}$, meeting $D_{12}$. Let $C^{+}$be the flopped curve. By Lemma 6.14, any flopping contraction in the factorisation of $\phi$ contracts an interior (-1)-curve, so $C$ is not contained in $\left(\operatorname{Sing} \mathcal{Y}_{c}\right) \cap Y_{1}$.

Suppose $C_{k}$ is not flopped by any $\mathcal{Y}_{k}^{\psi} \rightarrow \mathcal{Y}_{k+1}^{\psi}$ for any index $k$, with $\left(D_{12}\right)_{k} \cdot C_{k}=1$ and that the same is true for any $\psi$-flop of $C$ or any replacement. Suppose first that $C$ is an annex. Then $C$ is $D_{12}$-good and $\Gamma_{\left(Y_{1}\right)_{1}^{\phi}}$ is very degenerate. By Lemma $6.31, \Gamma_{\left(Y_{1}\right)_{n}^{\phi}}$ is very degenerate. By hypothesis, $\Gamma_{(Y)_{m}^{\psi}}$ is regular. But from Condition (6.1), we obtain $\left(Y_{1}\right)_{m}^{\psi} \stackrel{\sim}{\rightarrow}\left(Y_{1}\right)_{n}^{\phi}$, a contradiction. Hence, $C$ is not an annex. Thus we can apply Lemma 6.30 and the equality $\left(\phi_{*} D_{12}\right)^{2}=\left(\psi_{*} D_{12}\right)^{2}$, which follows from Condition (6.1), to conclude that either

(i) $C$ is not alone and a birational transform $C_{k}^{a}$ of the annex $C^{a}$ is the flopping curve of some $\mathcal{Y}_{k}^{\psi} \rightarrow \mathcal{Y}_{k+1}^{\psi}$, or

(ii) $C$ is not alone and there is a flop $C_{f}$ of $C$ that is replaced or reflected under $\phi$ or

(iii) $C$ is alone and there is a flop $C_{f}$ of $C$ that is replaced or reflected under $\phi$.

In cases (i) and (ii), $C$ is $D_{12}$-good. So by Lemma 6.26 , the case (ii) is impossible. In case (i), note that $C^{a}$ is $D_{21}$-good, and by the arguments in the proof of Example 6.20, any birational transform of $C^{a}$ meeting $\left(D_{2 j}\right)_{k}$ will be $D_{2 j}$-good for $j=1,3$. By the same argument as above, we obtain a contradiction to Condition (6.1) using Lemma 6.31. Hence we are in case (iii), namely $C$ is alone and there is a flop $C_{f}$ of $C$ that is replaced or reflected under $\phi$.

Let $D_{R}$ be the $R$-locus and let $Y_{R}$ be the component containing it. Let $q$ be the index of the replacement or reflection. Let $C^{R}$ be the replacement (or reflection) of $C_{f}$. The curve $C^{R}$ is $\left(D_{R}\right)_{q}$-good. This implies that exactly one of $\Gamma_{\left(Y_{R}\right)_{n}^{\phi}}$ and $\Gamma_{\left(Y_{R}\right)_{n}^{\psi}}$ is regular, again a contradiction to (6.1). Indeed, by Lemma 6.35, the $R$-locus is given by $D_{32}$. The implied curve structures are depicted in Figure 20. It then follows that in order to change the regularity of $Y_{R}$ under a flop $\psi_{i}$, there must be some $k$ such that $\psi_{k}$ has flopped curve $C_{k}$ or a $\psi$-flop of $C_{k}$, which we assumed is not the case. So this case also leads to a contradiction.

We conclude that there is an integer $p$ such that $\operatorname{Ex}\left(\phi_{p}\right)$ is a $\psi$-flop of $C$ with $\left(D_{12}\right)_{p} \cdot \operatorname{Ex}\left(\phi_{p}\right)=1$. We show that $p=0$. To get a contradiction, suppose $A_{\psi} \cdot C \geq 0$. In particular, $C$ is not an annex by Corollary 6.27. Suppose $p$ is chosen minimal. Lemma 6.22 implies that $C$ is replaced or reflected. It also follows that $C$ is $D_{12}$-good. Suppose there is no $i$ with $\operatorname{Ex}\left(\psi_{i}\right)=C_{i}, i<p$. Then the $R$-locus is $D_{31}$. Let $q$ be the index of 


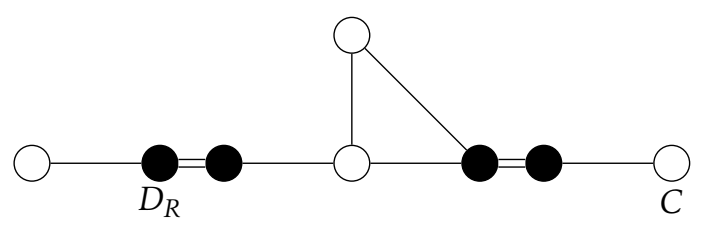

Figure 20. A subgraph of the augmented curve structures of $Y_{1}, Y_{2}$ and $Y_{3}$. The black vertices correspond to curves in the double locus of $\mathcal{Y}_{c}$. Here, the double lines between the black vertices indicates that the underlying curves are identified under the normalisation map.

the reflection/replacement. As before, we find that $\Gamma_{\left(Y_{3}\right)_{q}^{\psi}}$ and $\Gamma_{\left(Y_{3}\right)_{q+1}^{\psi}}$ are not both regular or very degenerate. By Lemma 6.31, $\Gamma_{\left(Y_{3}\right)_{m}^{\psi}}$ has the same regularity as $\Gamma_{\left(Y_{3}\right)_{q+1}^{\psi}}$. Note that there is a curve $E \in \Gamma_{Y_{1}}$ meeting $C$ and not meeting $D_{12}$ such that $E_{l}=\operatorname{Ex}\left(\psi_{l}\right)$ where $l+1$ is the minimal index such that $C_{l+1} \cdot\left(D_{13}\right)=1$. By $D_{12}$-goodness of $C$, there is no $i$ such that $E_{i}=\operatorname{Ex}\left(\phi_{i}\right)$ and $E_{i} \cdot\left(D_{13}\right)_{i}=1$. It follows that $\Gamma_{\left(Y_{3}\right)_{n}^{\phi}}$ has opposite regularity to $\Gamma_{\left(Y_{3}\right)_{m}^{\psi}}$. As above, this gives a contradiction. If there is $l<p$ with $\operatorname{Ex}\left(\psi_{l}\right)=C_{i}, i<p$, by Lemma 6.35 , the $R$-locus is $D_{23}$ and the same reasoning as before applies.

Corollary 6.37. Let $\mathcal{Y} \rightarrow S$ be a model of the DNV family of degree 2, with $\mathcal{Y}_{c}=\cup_{i} Y_{i}$. Suppose $\mathcal{Y}_{c} \in \operatorname{PMod}_{2}(\mathscr{P})$. Let $\phi$ and $\psi$ be birational automorphisms of $\mathcal{Y} \rightarrow$ S. Suppose

$$
\psi\left(Y_{i}\right) \subset Y_{j} \Leftrightarrow \phi\left(Y_{i}\right) \subset Y_{j} .
$$

Then there is a regular automorphism $\gamma$ of $\mathcal{Y}$ such that

$$
\psi=\gamma \circ \phi \text {. }
$$

Proof. We use the same notation as in the previous propositon. Let $A_{\phi}$ be negative on a curve $C_{1}$ generating an extremal ray. Then there is an $A_{\phi}$ flop $\phi_{1}: \mathcal{Y} \rightarrow \mathcal{Y}_{1}$ defined by contr $C_{C_{1}}$. This is also the $A_{\psi}$-flop defined by contr $C_{1}$, see e.g. [KM98, Definition 6.10]. Continuing this way, one obtains a sequence of flops factoring $\psi$ and $\phi$ up to isomorphism, i.e. a birational map $\psi_{n}: \mathcal{Y} \rightarrow \mathcal{Y}_{n}$ and isomorphisms $\alpha: \mathcal{Y}_{n} \rightarrow \mathcal{Y}$ and $\beta: \mathcal{Y}_{n} \rightarrow \mathcal{Y}$ such that $\psi=\alpha \circ \psi_{n}$ and $\phi=\beta \circ \psi_{n}$ and thus setting $\gamma=\alpha \circ \beta^{-1}$ we have $\psi=\gamma \circ \phi$.

Corollary 6.38. Let $\mathcal{Y} \rightarrow S$ be a model of the DNV family of degree 2, with $\mathcal{Y}_{c}=\cup_{i} Y_{i}$. Suppose $\mathcal{Y}_{c} \in \operatorname{PMod}(\mathscr{P})$. Let $\phi \in \operatorname{Bir}(\mathcal{Y} / S)$. Suppose $\phi\left(Y_{i}\right) \subset Y_{i}$ for all $i$. Then $\phi \in \operatorname{Aut}(\mathcal{Y} / S)$. In particular, $\operatorname{Bir}\left(\mathcal{Y}_{\mathscr{P}} / S\right)=\operatorname{Aut}\left(\mathcal{Y}_{\mathscr{P}} / S\right)$.

Proof. The first part follows by setting $\psi$ to be identity. The second part follows as any interior (-1)-curve $C$ on a component of $Y_{\mathscr{P}}$ is $D$-good for the component $D_{i j}$ of the double locus met by $C$. Indeed, let $\prod_{i=1}^{n} \phi_{i}$ be a factorisation of $\phi$ into flops and suppose there is an index $k$ such that $C_{k}=\operatorname{Ex}\left(\phi_{k}\right)$ for the birational transform of an interior $(-1)$ curve $C$ of a component of $Y_{\mathscr{P}}$. Obviously, we can assume $k=1$. By $D$-goodness, it follows from Lemma 6.30 that $\left(D_{j i}^{2}\right)_{t} \leq-2$ for all $t \geq 1$. This contradicts $\left(D_{i j}\right)_{n}^{2}=-1$.

Corollary 6.39. The automorphism group $\operatorname{Aut}\left(\mathcal{Y}_{\mathscr{P}} / S\right)$ of $\mathcal{Y}_{\mathscr{P}} / S$ contains a subgroup $S_{3}\left(\mathcal{Y}_{\mathscr{P}}\right)$ that is isomorphic to the symmetric group $S_{3}$ and acts faithfully by permutations on the set of components of $Y_{\mathscr{P}}$.

Proof. Let $C$ be an interior (-1)-curve on a component $Y_{\mathscr{P}, 1}$ of $Y_{\mathscr{P}}$, meeting $Y_{\mathscr{P}, 1} \cap Y_{\mathscr{P}, 2}$. It defines an elementary modification of type I, $Y_{\mathscr{P}} \rightarrow Y$. All components of $Y$ have non-degenerate curve structure, so there is a smoothing $\mathcal{Y}$ of $Y$ and a type I flop $\phi: \mathcal{Y}_{\mathscr{P}} \rightarrow \mathcal{Y}$ given by a flopping contraction contracting precisely $C$, by Proposition 6.6. Note that all components $Y_{i}$ of $Y$ have pairwise distinct curve structure. Assume that $Y_{\mathscr{P}, 1}$ is mapped to $Y_{1}$. In particular, they are not isomorphic. Let $C^{\prime}$ be an interior $(-1)$ curve different from $C$, on say $Y_{\mathscr{P}, k}$, meeting $Y_{\mathscr{P}, k} \cap Y_{\mathscr{P}, i}$ with $i \in\{1,2,3\} \backslash\{k\}$. Note that as $C^{\prime}$ is different from $C,(k, i) \neq(1,2)$. By the same argument as before, we obtain a type I flop $\phi_{(k, i)}: \mathcal{Y}_{\mathscr{P}} \rightarrow \mathcal{Y}^{\prime}$ given by a flopping contraction contracting precisely $C^{\prime}$. Let $Y_{k}^{\prime}$ be the the component which is the image of $Y_{\mathscr{P}, k}$. Now, $Y^{\prime}=\mathcal{Y}_{c}^{\prime}$ and $Y$ are isomorphic and hence by uniqueness of the DNV family and the fact that the 
generic fibres are isomorphic over $S$ there is an isomorphism $\gamma_{(k, i)}: \mathcal{Y} \rightarrow \mathcal{Y}^{\prime}$. Because the curve structures are distinct, necessarily $\gamma\left(Y_{1}\right)=Y_{k}^{\prime}$. It follows that there is a map $\psi_{(k, i)}$ and a commutative diagram of birational maps

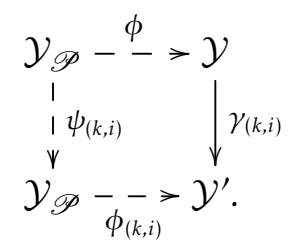

By Corollary $6.38, \psi_{(k, i)}$ is a morphism. By looking at curve structures, we find that $\psi_{(k, i)}$ maps $Y_{\mathscr{P}, 1}$ to $Y_{\mathscr{P}, k}$ and $Y_{\mathscr{P}, 2}$ to $Y_{\mathscr{P}, i}$. One finds that the possible combinations $(k, i)$ are $(1,3),(2,1),(2,3),(3,1)$, and $(3,2)$. Hence the permutations on the set of components of $Y_{\mathscr{P}}$ that are induced by automorphisms $\psi_{(k, i)}$ are $(1,3,2),(2,1,3),(2,3,1),(3,1,2)$ and $(3,2,1)$. Hence there is indeed a subgroup as claimed.

Definition 6.40. A model $Y \in \operatorname{PMod}_{2}$ is symmetric if there are distinct components $Y_{1}, Y_{3} \subset Y$ and an automorphism $\psi \in \operatorname{Aut}(Y)$ such that

$$
\psi_{\mid Y_{1}}: Y_{1} \stackrel{\sim}{\rightarrow} Y_{3} .
$$

If there $Y=\mathcal{Y}_{c}$ for a model $\mathcal{Y}$ of the DNV family, then $\mathcal{Y}$ is symmetric.

Note that this implies that $D_{13}^{2}=-1$, as $\psi\left(D_{13}\right)=D_{31}$. For models with symmetric central fibre, we have the following statement.

Proposition 6.41. Let $\mathcal{Y} \rightarrow S$ be a model of the DNV family of degree 2. Assume $\mathcal{Y}_{c} \in \operatorname{PMod}_{2}(\mathscr{P})$ is symmetric, but $\mathcal{Y} \neq \mathcal{Y}_{\mathscr{P}}$. Suppose $\phi, \psi$ are two birational automorphisms of $\mathcal{Y}$. Write $\mathcal{Y}_{c}=Y_{1} \cup Y_{2} \cup Y_{3}$ with notation such that $D_{13}^{2}=-1$. If $\phi\left(D_{13}\right)^{2}=\psi\left(D_{13}\right)^{2}$, then there is an automorphism $\gamma \in \operatorname{Aut}(\mathcal{Y} / S)$ such that

$$
\psi=\gamma \circ \phi
$$

Proof. As $\mathcal{Y} \neq \mathcal{Y}_{\mathscr{P}}$ it follows from $D_{13}^{2}=-1$ and symmetry that $Y_{1} \stackrel{\sim}{\rightarrow} Y_{3}$. It is straightforward to see, using $D_{13}$-goodness of interior $(-1)$ curves meeting $D_{13}$ or $D_{31}$, that $\phi\left(D_{13}\right)^{2}=-1$ implies that $\phi$ is regular, as then no curves can be flopped. Hence we can assume $\phi\left(D_{13}\right)^{2} \neq-1$. Also, note that any interior $(-1)$-curve $C$ meeting $D_{13}$ is $D_{13}$-good. More generally, it is easy to see that if

$$
\mathcal{Y} \rightarrow \mathcal{Y}_{1} \rightarrow \cdots \rightarrow \mathcal{Y}_{i} \stackrel{\phi_{i}}{\rightarrow} \mathcal{Y}_{i+1} \rightarrow \cdots \rightarrow \mathcal{Y}_{n} \stackrel{\sim}{\rightarrow} \mathcal{Y}
$$

is a factorisation of a birational automorphism of $\mathcal{Y}$ and $C$ is the flopping curve of $\mathcal{Y}_{k} \rightarrow \mathcal{Y}_{k+1}$, with $C \cdot\left(D_{13}\right)_{k}=1$, then $C$ is $\left(D_{13}\right)_{k}$-good.

We first show the proposition assuming $\Gamma_{Y_{1}}$ is regular. Note that then no flopping curve $C \subset\left(Y_{i}\right)_{k}$ in the factorisation can change the regularity of $\Gamma_{\left(Y_{i}\right)_{k}}$, as such a change would be irreversible by Lemma 6.31 . This implies the proposition. Indeed, there is neither replacement nor reflection as there are no regularity changing flops, so if $C$ is the flopping curve of some $\phi_{r}$, with $C \cdot\left(D_{i j}\right)_{r}=1$, we conclude from Lemma 6.30 and the condition on intersection numbers that for some $p, C_{p}$ is the flopping curve of some $\psi_{p}$, with $C_{p} \cdot\left(D_{i j}\right)_{p}=1$. As in the proof of Proposition 6.36, it follows that $p=0$. Arguing as in Corollary 6.37, the claim follows.

Now, suppose $\Gamma_{Y_{1}}$ is very degenerate. Then $\mathcal{Y}_{c}$ is uniquely defined up to isomomorphism: we have $D_{12}^{2}=D_{32}^{2}=4, D_{13}^{2}=D_{31}^{2}=-1, D_{21}^{2}=D_{23}^{2}=-6$ and also $\Gamma_{Y_{3}}$ is very degenerate. Also, both $\Gamma_{Y_{1}}$ and $\Gamma_{Y_{3}}$ have three vertices while $\Gamma_{Y_{2}}$ has 18 vertices. If $\phi$ is a birational automorphism such that $\phi\left(D_{13}\right)^{2} \neq-1$, then either $\phi\left(D_{13}\right)^{2}=4, \phi\left(D_{12}\right)^{2}=-1$ and $\phi\left(D_{23}\right)^{2}=4$ or $\phi\left(D_{13}\right)^{2}=-6, \phi\left(D_{12}\right)^{2}=-6$ and $\phi\left(D_{23}\right)^{2}=-1$. We first show that if

$$
\mathcal{Y} \rightarrow \mathcal{Y}_{1} \rightarrow \cdots \rightarrow \mathcal{Y}_{i} \stackrel{\phi_{i}}{\rightarrow} \mathcal{Y}_{i+1} \rightarrow \cdots \rightarrow \mathcal{Y}_{n} \stackrel{\sim}{\rightarrow} \mathcal{Y}
$$


is a factorisation of $\phi$, and $C$ the flopping curve of some $\phi_{k}$, then there is an exhaustive $C$-sequence $K_{C}^{\phi}$. By symmetry, it is enough to show this for the case $\phi\left(D_{13}\right)^{2}=4$. So let $C$ be the flopping curve of some $\phi_{k}$. Then $\left(D_{i j}\right)_{k} \cdot C=1$ for some $D_{i j}$. If $D_{i j}=D_{13}$ or $D_{i j}=D_{31}$, then, as remarked above, $C$ is $\left(D_{i j}\right)_{k}$-good. If $D_{i j}=D_{21}$, then it may be that the flopped curve $C^{+}$of $\phi_{k}$ is not alone. In that case however, both curves $C^{+}$and $C^{\prime}$ meeting $\left(D_{12}\right)_{k+1}$ are $\left(D_{12}\right)_{k+1}$-good by the same argument as in Example 6.20, so from the condition on the intersection numbers, there is no $\phi_{s}$ with flopping curve a birational transform of $C^{+}$or $C^{\prime}$. The same argument applies to $D_{23}$, impliying that $D_{i j}=D_{21}$ and $D_{i j}=D_{23}$ cannot happen. One concludes that there is an exhaustive $C$-sequence $K_{C}^{\phi}$.

To prove the proposition, assume there are factorisations of $\phi$ and $\psi$ that agree up to term $l$. As in Proposition 6.36 we let $A_{\phi}, A_{\psi}$ denote the bundles defining the sequences of flops. If $C$ is the flopping curve of $\phi_{l}$, with $C \cdot\left(D_{i j}\right)_{l}=1$, because of the existence of an exhaustive $C$ sequence $K_{C}^{\phi}$, the condition on intersection numbers implies that there is a flop $\psi_{s}$ with flopping curve $C_{s}^{\psi}$ : if $C$ is alone, this is immediate. If $C$ is not alone, then flopping the companion of $C$ implies that $\Gamma_{\left(Y_{i}\right)_{m}^{\psi}}$ and $\Gamma_{\left(Y_{i}\right)_{m}^{\phi}}$ have distinct regularity, a contradiction. Hence indeed there is a $\psi_{s}$ with the claimed properties. We show $s=l$.

Suppose first that $C$ is alone. Assume $\left(A_{\psi}\right)_{l} \cdot C_{l} \geq 0$. We can deduce from Corollary 6.27 that $C$ is alone and from Lemma 6.22 we get a minimal $q, s>q>l$ such that $C_{s}^{\phi} .\left(D_{i k}\right)_{q}^{\phi}=1, k \neq\{i, j\}$. Write $E=\operatorname{Ex}\left(\phi_{k}\right)$ for the flopping curve and $E^{+}$for the flopped curve. If $E$ is not replaced with $R$-locus $D_{k i}$ we have $C_{i} \cdot\left(D_{i k}\right)_{s}^{\phi}=1$ for all $i \geq q$, contradicting $C=\operatorname{Ex}\left(\psi_{s}\right)$. Hence $E^{+}$is not alone and, letting $E^{c}$ be the companion of $E^{+}$, there is a $t, s>t>q$ such that $\left(E^{c}\right)_{t}=\operatorname{Ex}\left(\psi_{t}\right)$ and $E_{t}^{c} \cdot\left(D_{k i}\right)_{t}=1$.

If $E^{+}$is an annex, it follows that the curve structure $\Gamma_{\left(Y_{i}\right)_{l}}$ consists of only two vertices and there is no $(-1)$-curve meeting $\left(D_{k i}\right)_{m}^{\phi}$ for any $m \geq s$. This implies that $\Gamma_{\left(Y_{i}\right)_{m}}$ is a singleton for $m \geq l+1$, a contradiction. If $E^{+}$is not an annex, then $\Gamma_{\left(Y_{k}\right)_{m}^{\phi}}$ is regular for all $m \geq l$ while $\Gamma_{\left(Y_{k}\right)_{m}^{\psi}}$ is very degenerate for $m>t$, a contradiction. Hence $\left(A_{\psi}\right)_{s} \cdot C<0$ and it follows inductively that $\phi$ and $\psi$ agree up to automorphism.

\subsection{Orbits}

Let $\mathcal{Y} \rightarrow S$ a be model of the DNV family of degree 2. We recall the action of $\operatorname{Bir}(\mathcal{Y} / S)$ on the Mori fan $\operatorname{MF}(\mathcal{Y} / S)$ in somewhat more detail. Let $\left(\mathcal{Y}^{\prime}, f\right)$ be a marked minimal model of the DNV family, i.e. a model $\mathcal{Y}^{\prime}$ of the DNV family together with a birational map $f: \mathcal{Y} \rightarrow \mathcal{Y}^{\prime}$. This determines a maximal cone $C(f)$ of $\operatorname{MF}(\mathcal{Y} / S)$, defined as the pullback under $f$ of the Nef cone of $\mathcal{Y}^{\prime}$.

The group $\operatorname{Bir}(\mathcal{Y} / S)$ acts on the cones of $\operatorname{MF}(\mathcal{Y} / S)$. Suppose $g: \mathcal{Y} \rightarrow \mathcal{Z}$ is another marked minimal model with $\mathcal{Z}$ isomorphic to $\mathcal{Y}^{\prime}$ via $h: \mathcal{Z} \rightarrow \mathcal{Y}^{\prime}$. We can replace $g$ by $h \circ g$ and assume $\mathcal{Z}=\mathcal{Y}^{\prime}$, as the corresponding cones are identical. Then $\gamma=f^{-1} \circ g$ is a birational $S$-automorphism of $\mathcal{Y}$ mapping $C(f)$ to $C(g)$. Hence the orbit of $C(f)$ under the action of $\operatorname{Bir}(\mathcal{Y} / S)$ is parameterised by the set of marked minimal models $(\mathcal{Z}, g)$ with $\mathcal{Z} \cong \mathcal{Y}^{\prime}$. If $f=\operatorname{id}_{\mathcal{Y} / S}$ is the identity on $\mathcal{Y}$, this trivially defines a model $\left(\mathcal{Y}\right.$, $\left.\mathrm{id}_{\mathcal{Y} / S}\right)$ and the corresponding cone is simply $\operatorname{Nef}(\mathcal{Y} / S)$.

Definition 6.42. Let $\mathcal{Y} \rightarrow S$ be a model of the Dolgachev-Nikulin-Voisin family of degree $2 d$. If $\sigma$ is a maximal cone in the orbit of $\operatorname{Nef}(\mathcal{Y} / S)$ under $\operatorname{Bir}(\mathcal{Y} / S)$ we say that $\sigma$ is a cone associated to $\mathcal{Y} \rightarrow S$.

Proposition 6.43. Let $\mathcal{Y} \rightarrow S$ be a model of the $D N V$ family of degree 2. Let $\sigma \in \operatorname{MF}(\mathcal{Y} / S)$ be an associated maximal cone and $\operatorname{Bir}(\mathcal{Y} / S) \cdot \sigma$ be the orbit of $\sigma$ under $\operatorname{Bir}(\mathcal{Y} / S)$. Then

$$
|\operatorname{Bir}(\mathcal{Y} / S) \cdot \sigma|= \begin{cases}1 & \text { if } \mathcal{Y}=\mathcal{Y}_{\mathscr{P}}, \\ 3 & \text { if } \mathcal{Y}_{c} \text { is symmetric, } \mathcal{Y} \neq \mathcal{Y}_{\mathscr{P}}, \\ 6 & \text { else. }\end{cases}
$$

Proof. We first assume $\mathcal{Y}_{c} \in \operatorname{PMod}_{2}(\mathscr{P})$. If $\mathcal{Y}=\mathcal{Y}_{\mathscr{P}}$, the result follows from Corollary 6.38 together with Corollary 6.39. In general, by Corollary 6.37, there are at most 5 birational automorphism that are not 
regular (up to composition with an automorphism). This follows from the fact that the fibre has 3 components and hence there are only 5 permutations of the components which are not the identity. Let $\mathcal{Y}$ be a model different from $\mathcal{Y}_{\mathscr{P}}$ and let $\phi: \mathcal{Y} \rightarrow \mathcal{Y}_{\mathscr{P}}$ be a birational map. For each $\sigma \in S_{3}$, let $g_{\sigma} \in \operatorname{Aut}\left(\mathcal{Y}_{\mathscr{P}}\right)$ be an automorphism permuting the components of the central fibre as in Corollary 6.39. One obtains 6 birational maps $\alpha_{\sigma}=\phi^{-1} \circ g_{\sigma} \circ \phi$. We show that if $\mathcal{Y}_{c}$ is not symmetric, 5 of the maps $\alpha_{\sigma}$ are not regular and distinct in the sense that for any pair $\alpha_{\sigma}, \alpha_{\sigma^{\prime}}$ with $\sigma \neq \sigma^{\prime}$, there does not exist an automorphism $\beta$ of $\mathcal{Y}$ with $\alpha_{\sigma}=\beta \circ \alpha_{\sigma^{\prime}}$. Suppose there is an $\alpha:=\alpha_{\sigma}$ that is regular and does not map each component of $\mathcal{Y}_{c}$ into itself. Regularity implies $\alpha$ is an isomorphism. We obtain that $\mathcal{Y}_{c}$ is symmetric. Indeed, $\alpha$ induces an automorphism of $\mathcal{Y}_{c}$ and by assumption, there is a component $\left(\mathcal{Y}_{c}\right)_{1}$ mapped to a component $\left(\mathcal{Y}_{c}\right)_{2}$, so $\mathcal{Y}_{c}$ is symmetric. Hence none of the $\alpha_{\sigma}$ is a regular map. Also, all the $\alpha_{\sigma}$ are distinct: as $\mathcal{Y}_{c}$ is not symmetric, all automorphisms fix the components of $\mathcal{Y}_{c}$, so by construction, no two $\alpha_{\sigma}$ can be related by an automorphism.

Now assume $\mathcal{Y}_{c}=Y_{1} \cup Y_{2} \cup Y_{3}$ is symmetric. By symmetry, there are two components, say $Y_{1}$ and $Y_{3}$, such that $Y_{1} \stackrel{\sim}{\rightarrow} Y_{3}$ and $D_{13}^{2}=D_{31}^{2}=-1$. By Proposition 6.41, up to automorphism, there are at most 2 birational automorphisms of $\mathcal{Y}$. Arguing as in the preceeding case, there are precisely 2 birational automorphisms up to automorphism, say $\phi$ and $\psi$, where we define $\phi$ as the map such that $\phi\left(D_{13}\right)^{2}=D_{21}^{2}$ and $\psi$ by requiring $\psi\left(D_{13}\right)^{2}=D_{12}^{2}$. These do not agree up to automorphism as the existence of a $\gamma \in \operatorname{Aut}(\mathcal{Y})$ with $\gamma \circ \psi=\phi$ implies $D_{12}^{2}=D_{21}^{2}=-1$ and thus $\mathcal{Y}=\mathcal{Y}_{\mathscr{P}}$.

Now suppose $\mathcal{Y}_{c} \in \operatorname{PMod}_{2}(\mathscr{T})$. Suppose first that the model is obtained by a single type II flop from $\mathcal{Y}_{\mathscr{P}}$. Then $\mathcal{Y}$ is symmetric and the orbit of the associated cone has length 3 . Second, if the type II flop of $\mathcal{Y}$ yields a model $\mathcal{Y}^{+}$not isomorphic to $\mathcal{Y}_{\mathscr{P}}$, still $\mathcal{Y}_{c}^{+} \in \operatorname{PMod}(\mathscr{P})$. Note that models obtained from $\mathcal{Y}^{+}$by applying different single type II flops to $\mathcal{Y}^{+}$are non-isomorphic. Hence, if $\sigma$ is an associated cone of $\mathcal{Y}$, there is a unique cone $\sigma^{+}$associated to $\mathcal{Y}^{+}$meeting $\sigma$ in codimension 1 and vice versa. It follows that if $\sigma$ and $\sigma^{+}$are associated cones of $\mathcal{Y}$ and $\mathcal{Y}^{+}$, the orbits have the same length, i.e. we have

$$
|\operatorname{Bir}(\mathcal{Y} / S) \cdot \sigma|=\left|\operatorname{Bir}(\mathcal{Y} / S) \cdot \sigma^{+}\right| \text {. }
$$

Also, $\mathcal{Y}$ is symmetric if and only if $\mathcal{Y}^{+}$is symmetric, as is easily checked by a direct calculation.

Remark 6.44. Suppose $\gamma \in \operatorname{Bir}(\mathcal{Y} / S)$ fixes the cone $C(f)$ (as a cone), i.e. $\gamma(C(f))=C(f)$. Then $\left(\mathcal{Y}^{\prime}, f \circ \gamma\right)$ is a marked minimal model that is isomorphic to $\left(\mathcal{Y}^{\prime}, f\right)$. In particular, there is $\beta$ in $\operatorname{Aut}\left(\mathcal{Y}^{\prime} / S\right)$ with $f \circ \gamma=\beta \circ f$, see e.g. [Kaw97, Lemma 1.5]. Conversely, setting $\gamma=f^{-1} \circ \beta \circ f$ for any $\beta \in \operatorname{Aut}\left(\mathcal{Y}^{\prime}\right)$ defines an element of the stabilizer $\operatorname{Bir}(\mathcal{Y} / S)_{C(f)}$ of $C(f)$.

Hence

$$
\gamma \mapsto f \circ \gamma \circ f^{-1}
$$

defines an isomomorphism

$$
\operatorname{Aut}\left(\mathcal{Y}^{\prime} / S\right) \rightarrow \operatorname{Bir}(\mathcal{Y} / S)_{C(f)}
$$

We next construct automorphisms of symmetric models of the DNV family that permute the smooth components of the central fibre.

Proposition 6.45. Let $\mathcal{Y}$ be a symmetric model of the DNV family of degree 2. Suppose $\mathcal{Y}_{c}=Y_{1} \cup Y_{2} \cup Y_{3}$ and let $\phi \in \operatorname{Aut}\left(\mathcal{Y}_{c}\right)$ be an automorphism with $\phi\left(Y_{2}\right)=Y_{3}$. Then there is an automorphism $\psi \in \operatorname{Aut}(\mathcal{Y} / S)$ of the total space such that $\psi\left(Y_{2}\right)=Y_{3}$ and $\psi\left(Y_{3}\right)=Y_{2}$.

Proof. If $\mathcal{Y} \stackrel{\sim}{\rightarrow} \mathcal{Y}_{\mathscr{P}}$ the proposition follows from Corollary 6.39 . Hence suppose that $\mathcal{Y}$ is not isomorphic to $\mathcal{Y}_{\mathscr{P}}$. We will first construct birational isomorphisms $\gamma_{i}, i=1,2,3$ of $\mathcal{Y}$ such that the orbit $\operatorname{Nef}(\mathcal{Y} / S)$ in $\operatorname{MF}(\mathcal{Y} / S)$ is $\left\{C\left(\gamma_{1}\right), C\left(\gamma_{2}\right), C\left(\gamma_{3}\right)\right\}$. For this we fix a birational map $\phi: \mathcal{Y} \rightarrow \mathcal{Y}_{\mathscr{P}}$. Write $Y_{\mathscr{P}}=Y_{1}^{\prime} \cup Y_{2}^{\prime} \cup Y_{3}^{\prime}$ with indices chosen such that $\phi\left(Y_{i}\right) \subset Y_{i}^{\prime}$. Let $g_{1}$ be the identity on $\mathcal{Y}_{\mathscr{P}}$ and choose elements $g_{i} \in$ $\operatorname{Aut}\left(\mathcal{Y}_{\mathscr{P}} / S\right), i=2,3$ with $g_{i}\left(Y_{1}^{\prime}\right)=Y_{i}^{\prime}$. These exist by Corollary 6.39 . We then define the maps $\gamma_{i}$ via the commutative diagrams 


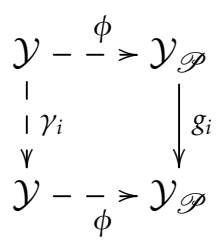

as $\gamma_{i}=\phi^{-1} \circ g_{i} \circ \phi$. In particular, $\gamma_{1}$ is the identity on $\mathcal{Y}$. Now, note that if $\mathcal{Y}_{c} \in \operatorname{PMod}(\mathscr{T})$ then any automorphism of $\mathcal{Y}$ maps the special component to itself as it permutes the smooth components. With our assumptions, the special component is $Y_{1}$. Similarly, if $\mathcal{Y}_{c} \in \operatorname{PMod}(\mathscr{P})$, any automorphism of $\mathcal{Y}$ maps $Y_{1}$ to itself, as $D_{23}^{2}=-1$ and $D_{12}^{2}=D_{21}^{2} \neq-1$ by symmetry. So in any event, since $\gamma_{i}$ maps $Y_{1}$ to $Y_{i}$ the maps $g_{2}$ and $g_{3}$ cannot be automorphisms of $\mathcal{Y}$.

We claim that the cones $C\left(\gamma_{i}\right)$ are pairwise distinct. Indeed, if $C\left(\gamma_{i}\right)=C\left(\gamma_{j}\right)$, there exists, by the above Remark 6.44 an automorphism $h \in \operatorname{Aut}(\mathcal{Y} / S)$ with $\gamma_{i}=h \circ \gamma_{j}$. We get

$$
h \circ \gamma_{j}\left(Y_{1}\right) \subset Y_{j} \text { and } \gamma_{i}\left(Y_{1}\right) \subset Y_{i}
$$

and hence $i=j$. By Proposition 6.43, the orbit of $\operatorname{Nef}(\mathcal{Y} / S)$ in $\operatorname{MF}(\mathcal{Y} / S)$ consists of three cones and is thus the set $\left\{C\left(\gamma_{1}\right), C\left(\gamma_{2}\right), C\left(\gamma_{3}\right)\right\}$. We now construct the desired automorphism $\psi$. Again by Corollary 6.39 there is an automorphism $g_{0} \in \operatorname{Aut}\left(\mathcal{Y}_{\mathscr{P}} / S\right)$ such that

$$
g_{0}\left(Y_{1}^{\prime}\right)=Y_{1}^{\prime}, g_{0}\left(Y_{2}^{\prime}\right)=Y_{3}^{\prime} \text { and } g_{0}\left(Y_{3}^{\prime}\right)=Y_{2}^{\prime} .
$$

Consider the map $\psi=\phi^{-1} \circ g_{0} \circ \phi$. Because $g_{0}$ fixes $Y_{1}^{\prime}$ it follows that also $\psi$ fixes $Y_{1}$. So we cannot have $C(\psi)=C\left(\gamma_{i}\right)$ for $i=2,3$ by the same reasoning as before. So necessarily $C(\psi)=C\left(\gamma_{1}\right)$. It follows now from Remark 6.44 that $\psi \in \operatorname{Aut}(\mathcal{Y} / S)$ and by construction $\psi$ permutes the components of $\mathcal{Y}$ as claimed.

\section{Counting models}

In this section we will count the elements of $\mathrm{PMod}_{2}$. We will first show that this can be done by counting triples of curve structures. The idea is to then start with the distinguished modes $Y_{\mathscr{T}}$ and $Y_{\mathscr{P}}$ and analyse in each case which type I flops are allowed under the condition that projectivity is preserved. This is a finite process and analysing it will finally lead us to our main result.

\subsection{Automorphisms}

We will need certain automorphisms of components of surfaces in $\operatorname{PMod}_{2}$. We first calculate the automorphism group of the central fibre $Y_{\mathscr{P}}$ of the model $\mathcal{Y}_{\mathscr{P}}$.

Write $Y=\varphi_{2}$, where $\biguplus_{2}$ is the weak del Pezzo surface of degree 2 defined in Construction 2.25. Observe that any automorphism $\gamma$ of $Y$ fixes the set of interior special points $\left\{p_{1}, p_{2}\right\}$ as it fixes the set of $(-1)$-curves. Hence $\gamma$ lifts to an automorphism of the (2,2)-blow-up $Y_{(2,2)}$ of $Y$ in $\left(p_{1}, p_{2}\right)$. Also, any automorphism of $Y_{(2,2)}$ fixes the set of exceptional curves of the blow-up $Y_{(2,2)} \rightarrow Y$, as these contain all (-1)-curves, and thus descends to $Y$. Applying [Loo81, Chapter I, Corollary 5.4] and [GHK15, Remark 5.2] to $Y_{(2,2)}$, it follows that $\operatorname{Aut}(Y)$ is a subgroup of the dihedral group $\mathbb{Z}_{2} \times \mathbb{Z}_{2}$. Indeed, we will show that $\operatorname{Aut}(Y)=\mathbb{Z}_{2} \times \mathbb{Z}_{2}$.

First, we give an alternative construction of $Y$. Let $Q=\mathbb{P}^{1} \times \mathbb{P}^{1}$. Let $\bar{D}=\bar{D}_{1}+\bar{D}_{2}+\bar{D}_{3}+\bar{D}_{4}$ be its toric boundary, ordered cyclically. Let $F_{1}$ be a fibre of the ruling with fibre $\bar{D}_{1}$ that meets $\bar{D}$ in smooth points of $\bar{D}$. Let $p_{2}$ be the point in $F_{1} \cap \bar{D}_{2}$ and $p_{4}$ be the point in $F_{1} \cap \bar{D}_{4}$. Similarly, let $F_{2}$ be a fibre of the ruling with fibre $\bar{D}_{2}$ that meets $\bar{D}$ in smooth points of $\bar{D}$ with $p_{1}$ the point in $F_{2} \cap \bar{D}_{1}$ and $p_{3}$ the point in $F_{2} \cap \bar{D}_{3}$, see Figure 21.

Let $\tilde{\pi}: \tilde{Y} \rightarrow Q$ be the $(1,3,1,3)$-blow-up of $Q$ in $\left(p_{1}, p_{2}, p_{3}, p_{4}\right)$. The birational transforms $\tilde{D}_{1}$ and $\tilde{D}_{3}$ of $\bar{D}_{1}$ and $\bar{D}_{3}$ respectively under $\tilde{\pi}^{-1}$ are $(-1)$-curves. Let $\tilde{Y} \rightarrow Y$ be the induced contraction. The surface $Y$ 


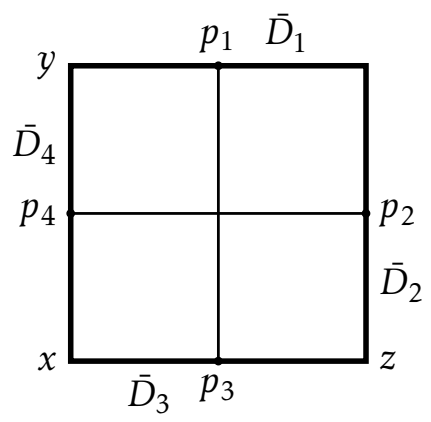

Figure 21. $\mathbb{P}^{1} \times \mathbb{P}^{1}$ with the points $q_{1}, q_{2}, p_{1}, p_{2}$.

is a weak del Pezzo surface of degree 2 with an $E_{6}$ root system of effective curves and thus isomorphic to $\bigcup_{2}$, see e.g. the global Torelli theorem in [GHK15].

Using this construction, it is straightforward to produce automorphisms on $Y$. First, being a product of two copies of $\mathbb{P}^{1}$, every pair $\left(\psi_{1}, \psi_{2}\right)$ of automorphisms of $\mathbb{P}^{1}$ induces an automorphism of $\mathbb{P}^{1} \times \mathbb{P}^{1}$. Let $y=\bar{D}_{1} \cap \bar{D}_{4}$ and $x=\bar{D}_{3} \cap \bar{D}_{4}$. Let $\bar{\phi}=\left(\phi_{1}, \mathrm{id}_{\mathbb{P}^{1}}\right)$ be the automorphism of $Q$ that is given on $\bar{D}_{4}$ by the automorphism $\phi_{1}$ of $\mathbb{P}^{1}$ defined by

$$
\begin{gathered}
x \mapsto y \\
y \mapsto x \\
p_{4} \mapsto p_{4},
\end{gathered}
$$

and by the identity on the second ruling. By the universal property of blow-ups, $\bar{\phi}$ lifts to an automorphism $\tilde{\phi}$ of $\tilde{Y}$. By construction, $\tilde{\phi}$ maps $\tilde{D}_{1}$ to $\tilde{D}_{3}$ and vice versa. Thus $\tilde{\phi}$ descends to an automorphism $\phi$ of $Y$. Note that $\phi$ acts as an involution on each of the components of the anticanonical divisor $D=D_{2}+D_{4}$ of $Y$.

We can do the same construction for an automorphism $\bar{\psi}=\left(\mathrm{id}_{\mathbb{P}^{1}}, \psi_{2}\right)$ of $Q$ with $\psi_{2}$ the automorphism of $\bar{D}_{3}$ with

$$
\begin{gathered}
x \mapsto z \\
z \mapsto x \\
p_{3} \mapsto p_{3},
\end{gathered}
$$

where $z$ is the point in $\bar{D}_{2} \cap \bar{D}_{3}$. This yields an automorphism $\psi$ of $Y$ interchanging the components $D_{2}$ and $D_{4}$. As we have seen that $\operatorname{Aut}(Y)$ is a subgroup of $\mathbb{Z}_{2} \times \mathbb{Z}_{2}$, it follows that in fact $\phi$ and $\psi$ generate $\operatorname{Aut}(Y)=\mathbb{Z}_{2} \times \mathbb{Z}_{2}$.

As a consequence, we have the following straightforward lemma.

Lemma 7.1. Let $Y$ be a contraction of an $(n, m)$ - blow-up of $\biguplus_{2}$ in the interior special points. Let $D$ be the strict transform of the anticanonical divisor of $\bigsqcup_{2}$.

(i) Let $D_{0}$ be a component of $D$. Then there is an involution $\phi^{Y}: Y \rightarrow Y$ which restricts to an involution on $D_{0}$ fixing the interior special point.

(ii) Suppose $D=D_{2}+D_{4}$ and $D_{2}^{2}=D_{4}^{2}$. Then there is an involution $\psi^{Y}: Y \rightarrow Y$ interchanging $D_{2}$ and $D_{4}$ while fixing the points in $D_{2} \cap D_{4}$.

Proof. The morphism $\phi$ lifts to any $(n, m)$ - blow-up of $\bigsqcup_{2}$ in the interior special points and maps any interior $(-1)$ curve to itself, so it descends to an automorphism of $Y$. Similarly, $\psi$ induces an automorphism $\psi^{Y}$ if $D$ has two components $D_{2}, D_{4}$ with $D_{2}^{2}=D_{4}^{2}$.

Lemma 7.2. Let $Y, Y^{\prime}$ be components of surfaces in $\operatorname{PMod}_{2}(\mathscr{P})$. Let $D=D_{1}+D_{2}$ and $D^{\prime}=D_{1}^{\prime}+D_{2}^{\prime}$ be the double loci. Let $\alpha: Y \stackrel{\sim}{\rightarrow} Y^{\prime}$ be an isomorphism, mapping $D$ to $D^{\prime}$. Then $\Gamma_{Y} \stackrel{\sim}{\rightarrow} \Gamma_{Y^{\prime}}$. 
Proof. Let $p_{1}$ and $p_{2}$ be the interior special points of $Y$ and $q_{1}, q_{2}$ be those of $Y^{\prime}$, assuming $q_{i} \in \alpha\left(D_{i}\right)$. The assumptions imply that $\Gamma_{Y}$ is regular (degenerate) if and only if $\Gamma_{Y^{\prime}}$ is regular (degenerate). Then the result follows as $D_{i}^{2}=\alpha\left(D_{i}\right)^{2}$.

Proposition 7.3. Let $Y, Y^{\prime}$ be components of surfaces in $\operatorname{PMod}_{2}$. Suppose the curve structures $\Gamma_{Y}, \Gamma_{Y^{\prime}}$ are isomorphic and of the same type (see Definition 4.10). Let $D, D^{\prime}$ be the double curves of $Y$ and $Y^{\prime}$ respectively. Then there is an isomorphism $Y \stackrel{\sim}{\rightarrow} Y^{\prime}$ which identifies $D$ and $D^{\prime}$.

Proof. It is enough to proof the proposition in the type $d_{2}$ case, as the proof also implies the $d_{1}$ and the $d_{4}$ cases, these being blow-ups or contractions in nodes of the double curves. Let $D=D_{1}+D_{2}$ and $D^{\prime}=D_{1}^{\prime}+D_{2}^{\prime}$. Write $D_{i}^{2}=n_{i}$ and $\left(D_{i}^{\prime}\right)^{2}=n_{i}^{\prime}$. As $\Gamma_{Y} \cong \Gamma_{Y^{\prime}}$, we can choose indices such that $n_{i}=n_{i}^{\prime}$. Let $p_{i}$ and $p_{i}^{\prime}$ be the interior special points. If $n_{i} \geq 0$, blow up $Y$ in $p_{i}$ until $n_{i}=-1$, and the same for $Y^{\prime}$. Write $\tilde{Y}$ and $\tilde{Y}^{\prime}$ for the blow-ups. These are $(n, m)$-blow-ups of $\biguplus_{2}$ and thus we obtain a diagram

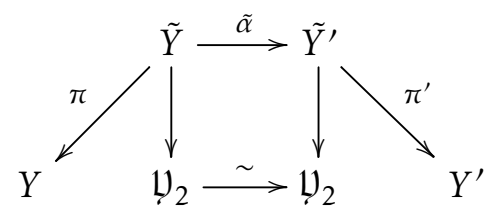

with $\tilde{\alpha}$ an isomorphism lifting the isomorphism $\underline{\varphi}_{2} \stackrel{\sim}{\rightarrow} \bigsqcup_{2}$, where we can assume that $\tilde{D}_{i}$ is mapped to $\tilde{D}_{i}^{\prime}$. Let $\Gamma$ and $\Gamma^{\prime}$ be the intersection graph of integral curves $C$ of $Y$ and $Y^{\prime}$ with $C^{2}<0$, and $C$ not a component of the double curve.

Then $\tilde{\alpha}$ identifies $\Gamma$ and $\Gamma^{\prime}$. Write $\pi^{\prime \prime}=\pi^{\prime} \circ \tilde{\alpha}$. Then the extremal cones of $\pi$ and $\pi^{\prime \prime}$ agree and hence there is an isomorphism $\alpha: Y \rightarrow Y^{\prime}$ mapping $D_{i}$ to $D_{i}^{\prime}$ and inducing an isomorphism $\Gamma_{Y} \rightarrow \Gamma_{Y^{\prime}}$, see e.g. [Deb01, Proposition 1.14].

For the following, recall our convention that for components $Y_{i}, Y_{j}$ of a semistable $\mathrm{K} 3$ surface, the self-intersection number of $Y_{i} \cap Y_{j}$ is calculated on $Y_{i}$.

Lemma 7.4. Let $\mathcal{Y}_{c}, \mathcal{Y}_{c}^{\prime}$ be in $\operatorname{PMod}_{2}(\mathscr{P})$. Write $\mathcal{Y}_{c}=Y_{1} \cup Y_{2} \cup Y_{3}$ and $\mathcal{Y}_{c}^{\prime}=Y_{1}^{\prime} \cup Y_{2}^{\prime} \cup Y_{3}^{\prime}$. Assume $D_{21}^{2} \neq D_{13}^{2}$. Suppose that there exist a permutation $\sigma \in \mathcal{S}_{3}$ and isomorphisms of curve structures $\alpha_{i}^{\Gamma}: \Gamma_{Y_{i}} \stackrel{\sim}{\rightarrow} \Gamma_{Y_{\sigma(i)}^{\prime}}$ such that $\left(Y_{\sigma(1)}^{\prime} \cap Y_{\sigma(2)}^{\prime}\right)^{2}=D_{12}^{2}$. Then there is an isomorphism $\alpha: \mathcal{Y}_{c} \stackrel{\sim}{\rightarrow} \mathcal{Y}_{c}^{\prime}$.

Proof. By Proposition 7.3, for each $\alpha_{i}^{\Gamma}$ there is an induced isomorphism $\alpha_{i}: Y_{i} \rightarrow Y_{\sigma(i)}^{\prime}$. We write $t_{1}, t_{2}$ for the triple points of $Y$. The interior special point contained in a component $D_{i j} \subset Y_{i}$ is denoted by $p_{i j}$. We use $t_{1}^{\prime}, t_{2}^{\prime}$ and $p_{i j}^{\prime}$ for the triple points and interior special points on $Y^{\prime}$, where we assume $\alpha_{1}\left(t_{i}\right)=t_{i}^{\prime}$. We have $\alpha_{1}\left(p_{12}\right)=p_{12}^{\prime}$ and as $\left(Y_{\sigma(1)}^{\prime} \cap Y_{\sigma(2)}^{\prime}\right)^{2}=D_{12}^{2}$, using Lemma 7.1, we can find an isomorphism $\alpha_{2}: Y_{2} \rightarrow Y_{\sigma(2)}^{\prime}$ with $\alpha_{2}\left(p_{21}\right)=p_{21}^{\prime}, \alpha_{2}\left(p_{23}\right)=p_{23}^{\prime}, \alpha_{2}\left(t_{1}\right)=t_{1}^{\prime}$ and $\alpha_{2}\left(t_{2}\right)=t_{2}^{\prime}$, by the (proof of) Proposition 7.3. Similarly, there is an isomorphism $\alpha_{3}: Y_{3} \rightarrow Y_{\sigma(3)}^{\prime}$ with $\alpha_{3}\left(p_{31}\right)=p_{31}^{\prime}, \alpha_{3}\left(p_{32}\right)=p_{32}^{\prime}, \alpha_{3}\left(t_{1}\right)=t_{1}^{\prime}$, and $\alpha_{3}\left(t_{2}\right)=t_{2}^{\prime}$. By construction, the $\alpha_{i}$ glue to an isomorphism $\alpha: Y \stackrel{\sim}{\rightarrow} Y^{\prime}$.

The following proposition says that in order to count models, it will be enough to count curve structures. We will first formulate this for models of class $\mathscr{P}$ and then extend it to models of class $\mathscr{T}$.

Proposition 7.5. Let $\mathcal{Y}, \mathcal{Y}^{\prime}$ be two models of the DNV family of degree 2 with $\mathcal{Y}_{c}, \mathcal{Y}_{c}^{\prime}$ in $\operatorname{PMod}_{2}(\mathscr{P})$ and components $Y_{i}$ and $Y_{i}^{\prime}$ respectively. Then $\mathcal{Y}$ and $\mathcal{Y}^{\prime}$ are isomorphic if and only if there are isomorphisms of curve structures $\Gamma_{Y_{i}} \rightarrow \Gamma_{Y_{\sigma(i)}^{\prime}}$ for some permutation $\sigma \in \mathcal{S}_{3}$.

Proof. It is enough to show that the central fibres are isomorphic, as then the models are isomorphic by uniqueness of the DNV family. 
Either both $\mathcal{Y}$ and $\mathcal{Y}^{\prime}$ are isomorphic to $\mathcal{Y}_{\mathscr{D}}$, or there is a component $Y_{1} \subset \mathcal{Y}_{c}$ such that for the components of the double curve $D=D_{12}+D_{13}$ of $Y_{1}, D_{12}^{2} \neq D_{13}^{2}$. There is a component $Y_{\sigma(1)}^{\prime}$ with $\Gamma_{Y_{1}} \stackrel{\sim}{\rightarrow} \Gamma_{Y_{\sigma(1)}^{\prime}}$ and by Proposition 7.3 an isomorphism $\alpha_{1}: Y_{1} \stackrel{\sim}{\rightarrow} Y_{\sigma(1)}^{\prime}$.

Let $Y_{2}$ be the component with $Y_{1} \cap Y_{2}=D_{12}$. We also have a component $Y_{\sigma(2)}^{\prime}$ with $\Gamma_{Y_{2}} \stackrel{\sim}{\rightarrow} \Gamma_{Y_{\sigma(2)}^{\prime}}$ and an isomophism $\alpha_{2}: Y_{2} \stackrel{\sim}{\rightarrow} Y_{\sigma(2)}^{\prime}$.

Consider first the case $\left(Y_{\sigma(1)}^{\prime} \cap Y_{\sigma(2)}^{\prime}\right)^{2}=D_{13}^{2}$. Then, as $D_{12}^{2} \neq D_{13}^{2}$ and we have isomorphisms of curve structures $\Gamma_{Y_{i}} \rightarrow \Gamma_{Y_{\sigma(i)}^{\prime}}$, we have $D_{31}^{2}=D_{23}^{2}$ and also $D_{23}^{2}=D_{13}^{2}$. Also, there is an isomorphism $\alpha_{3}: Y_{3} \stackrel{\sim}{\rightarrow} Y_{\sigma(3)}^{\prime}$ and $\left(Y_{\sigma(1)}^{\prime} \cap Y_{\sigma(3)}^{\prime}\right)^{2}=D_{12}^{2}$. By the same token, we obtain $D_{32}^{2}=D_{21}^{2}$ and $D_{23}^{2}=D_{12}^{2}$. We deduce that $\mathcal{Y}_{c}$ is given by three components $Y_{1}, Y_{2}$ and $Y_{3}$ with $D_{21}^{2}=D_{13}^{2}=D_{32}^{2}=a$ and $D_{12}^{2}=D_{31}^{2}=D_{23}^{2}=-a-2$ for some integer $a$. As $\Gamma_{Y_{1}}$ is non-degenerate, $a \leq 1$ and $-a-2 \leq 1$. Thus, by Proposition 4.19, all $Y_{i}$ are isomorphic and have indeed isomorphic curve structures. It is then immediate to choose an isomorphism $Y_{i} \rightarrow Y_{i}^{\prime}$ that induces an isomorphism $\mathcal{Y}_{c} \stackrel{\sim}{\rightarrow} \mathcal{Y}_{c}^{\prime}$. Hence $\mathcal{Y} \cong \mathcal{Y}^{\prime}$ by uniqueness of the DNV family.

In the remaining case, $\left(Y_{\sigma(1)}^{\prime} \cap Y_{\sigma(2)}^{\prime}\right)^{2}=D_{12}^{2}$. Then the result follows from Lemma 7.4. The other direction follows from Lemma 7.2.

We now consider models of class $\mathscr{T}$.

Proposition 7.6. Let $\mathcal{Y}, \mathcal{Y}^{\prime} \in \operatorname{PMod}_{2}(\mathscr{T})$ be two models of the DNV family of degree 2 with components $Y_{i}$ and $Y_{i}^{\prime}$ respectively. Then $\mathcal{Y}$ and $\mathcal{Y}^{\prime}$ are isomorphic if and only if there are isomorphisms of curve structures $\Gamma_{Y_{i}} \rightarrow \Gamma_{Y_{\sigma(i)}^{\prime}}$ for some permutation $\sigma \in \mathcal{S}_{3}$.

Proof. We first assume that there are isomorphisms of curve structures $\Gamma_{Y_{i}} \rightarrow \Gamma_{Y_{\sigma(i)}^{\prime}}$ for some permutation $\sigma \in \mathcal{S}_{3}$. We first note that there are models $\hat{\mathcal{Y}}, \hat{\mathcal{Y}}^{\prime}$ of class $\mathscr{P}$ and type II flops $\hat{\mathcal{Y}} \rightarrow \mathcal{Y}$ and $\hat{\mathcal{Y}}^{\prime} \rightarrow \mathcal{Y}^{\prime}$. Write $\hat{\mathcal{Y}}_{c}=\hat{Y}_{1} \cup \hat{Y}_{2} \cup \hat{Y}_{3}$ and $\hat{\mathcal{Y}}_{c}^{\prime}=\hat{Y}_{1}^{\prime} \cup \hat{Y}_{2}^{\prime} \cup \hat{Y}_{3}^{\prime}$ for the components of the central fibres. The curve structures of the surfaces $\hat{Y}_{i}$ and $\hat{Y}_{i}^{\prime}$ are determined by the curve structures of $Y_{i}$ and $Y_{i}^{\prime}$. This follows as any sequence of type I flops $\mathcal{Y}_{\mathscr{T}} \rightarrow \mathcal{Y}$ induces a sequence $\mathcal{Y}_{\mathscr{P}} \rightarrow \hat{\mathcal{Y}}$ and similar for $\mathcal{Y}^{\prime}$ and $\hat{\mathcal{Y}}^{\prime}$. Hence there exist a permutation $\tau \in \mathcal{S}_{3}$ and isomorphisms of curve structures $\Gamma_{\hat{Y}_{i}} \rightarrow \Gamma_{\hat{Y}_{\tau(i)}^{\prime}}$. Then, Proposition 7.5 implies that $\hat{\mathcal{Y}} \cong \hat{\mathcal{Y}}^{\prime}$. So $\mathcal{Y}$ and $\mathcal{Y}^{\prime}$ are both flops of the extremal contraction $\hat{\mathcal{Y}} \rightarrow \mathcal{Z}$ defining the type II flop, and hence isomorphic.

We now prove the remaining direction. So assume $\mathcal{Y} \stackrel{\sim}{\rightarrow} \mathcal{Y}^{\prime}$ via some isomorphism $h$. Any isomorphism preservers the number of $(-1)$-curves meeting a component of the double locus $D_{i j}$ and also the selfintersection numbers of the $D_{i j}$. But this datum uniquely determines curve structures of type $d_{4}$ and $d_{2}$. Hence we can find a permutation $\sigma \in \mathcal{S}_{3}$ and isomorphisms of curve structures $\Gamma_{Y_{i}} \rightarrow \Gamma_{Y_{\sigma(i)}^{\prime}}$.

\subsection{Counting models of class $\mathscr{T}$}

We now count the number of models of class $\mathscr{T}$. Our approach is as follows: we can count the number of models of class $\mathscr{T}$ by starting with $Y_{\mathscr{T}}$ and applying all possible elementary modifications of type I such that the flopped curve does not meet the singular locus of the special component. By Proposition 5.1, any surface obtained in this way is the central fibre of a model of the DNV family of degree 2. By Corollary 6.33, all models can be obtained in this way, up to isomorphism. By Proposition 7.6, the isomorphism classes are uniquely determined by curve structures. Hence, to count models we will count the distinct curve structures that one can obtain from $\mathcal{Y}_{\mathscr{T}}$ by type I elementary modifications. Note that by Remark 3.7, this is a finite problem. This counting of flops is then essentially reduced to counting possible combinations of self-intersection numbers of the nodal components of the double locus.

So let $Y \in \operatorname{PMod}_{2}(\mathscr{T})$. Let $\left(n_{1}, n_{2}\right)$ be the self intersection numbers of the preimages of the nodal components of the double curve - now written as $D_{1}, D_{2}-$ of the special component $Y_{\omega}$ of $Y$ on its normalisation. Let $Y_{i}$ be the smooth component glued to $D_{i}$. We will break up the analysis into several 


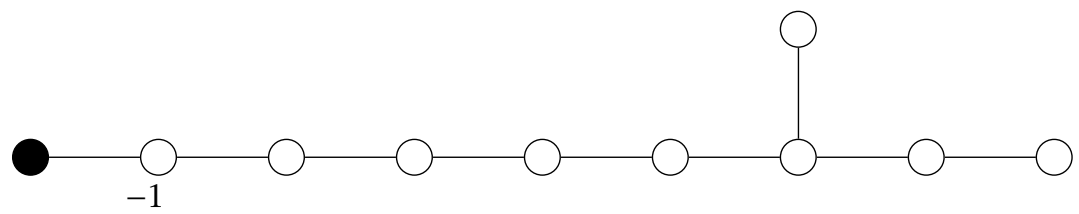

Figure 22. The augmented curve structure of a smooth component $Y$ of $Y_{\mathscr{T}}$. The black vertices correspond to the anticanonical divisor. All unlabelled curves of $\Gamma_{Y}$ have self intersection -2 .

cases, namely (i) $n_{1} \geq-1, n_{2} \leq-1$, (ii) $n_{1}, n_{2} \geq 0$ and (iii) $n_{1}, n_{2} \leq-2$. By the symmetry of $Y_{\mathscr{T}}$, these are all cases.

Consider first the case $n_{1} \geq-1$ and $n_{2} \leq-1$. The possible numerical combinations are as follows.

- $n_{1} \in\{-1,0,1\}$ and $n_{2} \in\{-1, \ldots,-9\}$. Note that there are two models with with $n_{2}=-8$ for any $n_{1}$. This is because after flopping 7 curves from a smooth component $Y_{i}$ of $Y_{\mathscr{T}}$, there are 2 interior $(-1)$-curves that can be flopped, $c f$. Figure 22. This gives 30 models.

- There are two different ways to obtain $n_{1}=2$, as after contracting 2 curves to obtain $n_{1}=1$, one can either contract the transform of a fork or a transform of a (-1)-curve on $Y_{\omega}$ meeting $D_{2}$. In the first case one can flop $r_{1} \in\{0, \ldots, 9\}$ curves from $Y_{2}$, with 2 choices for flopping 7 curves as above. In the second case one has to flop one curve from the smooth component $Y_{1}$ in order to obtain $n_{1}=2$. So one can flop $r_{2} \in\{0, \ldots, 8\}$ curves, with 2 possibilities for $r_{2}=6$. This gives $21=11+10$ models.

- $n_{1} \in\{3, \ldots, 9\}$. To obtain $n_{1}$, one needs to flop $n_{1}-1$ curves from $Y_{2}$ to $Y_{\omega}$ and then flop $n_{1}+1$ curves to $Y_{1}$. One can also flop more curves from $Y_{2}$ to $Y_{\omega}$ : for given $n_{1}$, one can flop $r \in\left\{0, \ldots, 9-n_{1}\right\}$ additional curves. If $n_{1}<9$, one value of $n_{2}$ can be obtained in two different ways, as above. This yields 34 models.

Hence we find $30+21+34=85$ models. Now, if both $n_{1}$ and $n_{2}$ are $\geq 0$, there is only the possibility $\left(n_{1}, n_{2}\right)=(0,0)$.

If both $n_{1}$ and $n_{2}$ are $\leq-2$, all models are obtained by flopping curves from the smooth components to the special component. These operations are independent on each $D_{i}$, so the models are given by intersection numbers

$$
\left(n_{1}, n_{2}\right) \in\{-2, \ldots,-9\}^{2},
$$

with two possibilities to obtain a -8 for each entry. This gives 81 models. More precisely, these models are paremetrised by the set

$$
\left(n_{1}, n_{2}\right) \in\left\{-2, \ldots,-8,-8^{\prime},-9\right\}^{2},
$$

Among these 81 there are the 9 models $(x, x)$ with $x \in\left\{-2, \ldots,-7,-8,-8^{\prime},-9\right\}$. Taking these out, the remaining set is given by $\left(n_{1}, n_{2}\right)$ with $n_{1} \neq n_{2}$, giving 72 models. Taking out the ordering of the components, we obtain 36 non-isomorphic models in this set. Adding up, we have $131=85+1+9+36$ projective models of class $\mathscr{T}$.

Theorem 7.7. There are 131 surfaces in $\operatorname{PMod}_{2}(\mathscr{T})$.

Note that the number of surfaces in the theorem is the number of orbits of maximal cones in the Mori fan under the action of the birational goup.

\subsection{Counting models of class $\mathscr{P}$}

We first count models such that at least one component of the central fibre has very degenerate curve structure.

Theorem 7.8. There are 104 surfaces $Y_{c}=Y_{1} \cup Y_{2} \cup Y_{3}$ in $\operatorname{PMod}_{2}(\mathscr{P})$ such that there is a component $Y_{i}$ with very degenerate curve structure. Explicitly, these are given as follows: 
(i) Surfaces $Y_{c}$ such that $\Gamma_{Y_{1}}$ is very degenerate, $\Gamma_{Y_{2}}$ is non-degenerate and $\Gamma_{Y_{3}}$ is tamely degenerate. There are 71 such models.

(ii) Surfaces $Y_{c}$ such that $\Gamma_{Y_{1}}$ is very degenerate, $\Gamma_{Y_{2}}$ is non-degenerate and $\Gamma_{Y_{3}}$ is very degenerate. There are 8 such models.

(iii) Surfaces $Y_{c}$ such that $\Gamma_{Y_{1}}$ is very degenerate, $\Gamma_{Y_{2}}$ is non-degenerate and $\Gamma_{Y_{3}}$ is non-degenerate. There are 25 such models.

Proof. Using Proposition 5.2, we see that at least one component of $Y_{c}$ has non-degenerate curve structure, so the three cases in the theorem exhaust all possible configurations of curve structures with at least one of the structures very degenerate: by assumption, one of the curve structures is very degenerate, so a second one has to be non-degenerate and the third one is as in the statement of the theorem. Also, by Proposition 4.19, after fixing regularity, it is enough to consider the self-intersection numbers of the double curves $D_{i j}$. Now, recall from Lemma 4.23 that there is a unique vertex $v_{D_{12}}$ meeting $D_{12}$, by regularity. If $Y_{c}$ is a model as in (i), then $v_{D_{12}} \cdot D_{12}=1$ or $v_{D_{12}} \cdot D_{12}=2$. In the first case, the conditions on the curve structures imply, using Proposition 4.19, that $D_{13}^{2}=4$ since $\Gamma_{Y_{1}}$ is very degenerate, $D_{21}^{2} \in[-3,1]$ since $\Gamma_{Y_{1}}$ is very degenerate and $\Gamma_{Y_{2}}$ is non-degenerate, and $D_{32}^{2} \in[2,10]$ by non-degeneracy of $\Gamma_{Y_{2}}$ and the fact that $\Sigma_{i}\left|\Gamma_{Y_{i}}\right|=24$. Projectivity of $Y_{c}$ further implies $D_{21}^{2}<1$, by Proposition 5.4. Conversely, by the results in subsection 5.2, each triple $\left(D_{13}^{2}, D_{21}^{2}, D_{32}^{2}\right)$ with $D_{13}^{2}=4, D_{21}^{2} \in[-3,0]$ and $D_{32}^{2} \in[2,10]$ determines a projective model, and these are pairwise non-isomorphic as the intersection numbers of the double curves are distinct. Hence there are 36 models with this specification of curve structures and $v_{D 12} \cdot D_{12}=1$.

If $v_{D 12} \cdot D_{12}=2$, we have $D_{12}^{2}=4$. Assume first that $D_{31}^{2} \geq 2$. From this condition, non-degeneracy of $Y_{2}$, and as $\Gamma_{Y_{3}}$ is regular, we have $D_{23}^{2} \in[-4,1]$ and counting possible flops, $D_{31}^{2} \in\left[2,6+D_{23}^{2}\right]$. Projectivity requires $D_{23}^{2}<1$ by Proposition 5.4, so one obtains 15 distinct models. Now suppose $D_{31}^{2}<2$. Then $D_{13}^{2} \in[-3,1]$ and $D_{32}^{2} \in\left[2,6+D_{13}^{2}\right]$, giving 20 models.

If $Y_{c}$ is a model as in (ii), we can assume $v_{D_{12}} \cdot D_{12}=2$. Then either $v_{D_{31}} \cdot D_{31}=2$ or $v_{D_{31}} \cdot D_{31}=1$. In the first case, $D_{12}^{2}=D_{32}^{2}=4$. One finds that up to isomorphism, we have $D_{13}^{2} \in[-3,0]$, so there are 4 models in this case. In the second case, $D_{31}^{2}=4, D_{12}^{2}=4$ and $D_{23}^{2} \in[-3,0]$ by non-degeneracy and projectivity. Hence there are again 4 such models.

If $Y_{c}$ is a model as in (iii), we can assume $v_{D_{23}} \cdot D_{23}=2$. Then $D_{13}=4, D_{12} \in[-3,1]$ and $D_{23} \in[-3,1]$. These models are pairwise non-isomorphic as the curve structure of $\Gamma_{Y_{3}}$ always has an exceptional vertex $v$ with $|L(v)|=9$. This never occurs for $\Gamma_{Y_{2}}$. One finds 25 models.

We now count models $Y \in \operatorname{PMod}_{2}(\mathscr{P})$ such that $\Gamma_{Y_{i}}$ is regular for all components $Y_{i} \subset Y$. We use the following shorthand notation. Fix a numbering of the components of $Y_{\mathscr{P}}$. There is a sequence of type I modifications $Y_{\mathscr{P}} \rightarrow Y$. Write $Y=\cup_{i} Y_{i}$, assuming that the $i$-th component of $Y_{\mathscr{P}}$ maps to $Y_{i}$. Let $D_{i}=Y_{i} \cap Y_{i+1}$, with $i=1,2,3$, indices considered modulo 4, considered as curve on $Y_{i}$. We have $D_{i}^{2}=-1+n_{i}$ for some $n_{i} \in \mathbb{Z}$.

Note that if $\Gamma_{Y_{i}}$ is regular for $i=1,2,3$, the triple $\left(n_{1}, n_{2}, n_{3}\right)$ determines $Y$ uniquely, by Proposition 7.5. The meaning of $\left(n_{1}, n_{2}, n_{3}\right)$ is that $n_{i}$ curves are flopped from $Y_{i}$ to $Y_{i+1}$ (or from $Y_{i+1}$ to $Y_{i}$, if $n_{i}<0$ ).

In order to simplify the arguments below, we now define certain operations on triples of integers.

Definition 7.9. Let $(x, y, z)$ be a triple. We shall call the triples $(z, x, y)$ and $(y, z, x)$ the shifts of $(x, y, z)$. The triple $(-y,-x,-z)$ will be called the involution of $(x, y, z)$. We write $(x, y, z) \sim(u, v, w)$ if the triples $(x, y, z)$ and $(u, v, w)$ are related by a sequence of shifts and involutions and call $(x, y, z)$ and $(u, v, w)$ equivalent.

Remark 7.10. We let $s:(x, y, z) \mapsto(z, y, x)$ be the shift operator and $\imath:(x, y, z) \mapsto(-y,-x,-z)$ be the involution operator. Then $\imath \circ s \circ \imath(x, y, z)=s^{2}(x, y, z)$ and $\imath \circ s^{2} \circ \imath(x, y, z)=s(x, y, z)$. Also, $s \circ \imath \circ s=\imath$ so any sequence of shifts and involutions reduces to $\imath^{a} \circ s^{b}$ or $s^{b} \circ \imath^{a}$ with $a \in\{0,1\}$ and $b \in\{0,1,2\}$. In particular, the group generated by $\imath$ and $s$ is isomorphic to $S_{3}$. 
Lemma 7.11. Let $Y, Y^{\prime}$ be two models in $\operatorname{PMod}_{2}(\mathscr{P})$ such that all curve structures $\Gamma_{Y_{i}}$ and $\Gamma_{Y_{i}^{\prime}}$ are regular, with triples $\left(n_{1}, n_{2}, n_{3}\right)$ and $\left(n_{1}^{\prime}, n_{2}^{\prime}, n_{3}^{\prime}\right)$ (relative to a chosen numbering of the components of $\left.Y_{\mathscr{P}}\right)$. Then $Y \cong Y^{\prime}$ if and only if $\left(n_{1}, n_{2}, n_{3}\right) \sim\left(n_{1}^{\prime}, n_{2}^{\prime}, n_{3}^{\prime}\right)$.

Proof. Write $Y=\cup Y_{i}$ and $Y^{\prime}=\cup Y_{i}^{\prime}$ for the central fibres. If $Y$ and $Y^{\prime}$ are isomorphic, after possible renumbering, we can assume $Y_{1} \cong Y_{1}^{\prime}$. Let $D^{\prime}=D_{1}^{\prime}+D_{2}^{\prime}$ be the anticanonical cycle on $Y_{1}^{\prime}$ with numbering chosen such that $D_{1} \cong D_{1}^{\prime}$, with $D_{1}=Y_{1} \cap Y_{2}$ and write $\left(D_{1}^{\prime}\right)^{2}=-1+x$ and $\left(D_{2}^{\prime}\right)^{2}=-1+y$. Then either the tuple $(-y, x)$ or the tuple $(-x, y)$ is contained in a shift of $\left(n_{1}^{\prime}, n_{2}^{\prime}, n_{3}^{\prime}\right)$. Let $Y_{2}^{\prime}$ be the component of $Y^{\prime}$ meeting $Y_{1}^{\prime}$ in $D_{2}^{\prime}$ and let $D^{\prime \prime}$ denote the component of the anticanonical cycle on $Y_{2}^{\prime}$ that is not glued to $Y_{1}^{\prime}$. Write $\left(D^{\prime \prime}\right)^{2}=-1+z$. It follows that after applying shifts, either $\left(n_{1}^{\prime}, n_{2}^{\prime}, n_{3}^{\prime}\right) \sim(-z,-y, x)$ or $\left(n_{1}^{\prime}, n_{2}^{\prime}, n_{3}^{\prime}\right) \sim(-x, y, z)$. Because $Y_{1} \cong Y_{1}^{\prime}$, we have $x=-n_{3}, y=n_{1}$ and $z=n_{2}$, thus we conclude that $\left(n_{1}^{\prime}, n_{2}^{\prime}, n_{3}^{\prime}\right) \sim\left(-n_{2},-n_{1},-n_{3}\right)$ or $\left(n_{1}^{\prime}, n_{2}^{\prime}, n_{3}^{\prime}\right) \sim\left(n_{3}, n_{1}, n_{2}\right)$. So indeed $\left(n_{1}, n_{2}, n_{3}\right) \sim\left(n_{1}^{\prime}, n_{2}^{\prime}, n_{3}^{\prime}\right)$.

If conversely the triples are equivalent, the corresponding models are isomorphic: let $(x, y, z)$ be a triple defining a model $Y=Y_{1} \cup Y_{2} \cup Y_{3}$. By our convention, this is the model where $x$ curves are flopped from $Y_{1}$ to $Y_{2}, y$ curves are flopped from $Y_{2}$ to $Y_{3}$ and $z$ curves are flopped from $Y_{3}$ to $Y_{1}$. The shift $(z, x, y)$ also defines a model, say $Y^{\prime}=Y_{1}^{\prime} \cup Y_{2}^{\prime} \cup Y_{3}^{\prime}$. Then $Y_{1}$ and $Y_{2}^{\prime}$ are obtained from $\bigcup_{2}$ by the same type I modifications and thus there is an isomorphism $Y_{1} \rightarrow Y_{2}^{\prime}$. Similarly there are isomorphisms $Y_{2} \rightarrow Y_{3}^{\prime}$ and $Y_{3} \rightarrow Y_{1}^{\prime}$. Thus $Y \stackrel{\sim}{\rightarrow} Y^{\prime}$ by Proposition 7.5. The case of an involution is similar.

Theorem 7.12. There are $27+103+225=353$ surfaces $Y=Y_{1} \cup Y_{2} \cup Y_{3}$ in $\operatorname{PMod}_{2}(\mathscr{P})$ such that the curve structures $\Gamma_{Y_{i}}$ are all regular. Explicitly, these are, up to equivalence, given as follows:

(i) Surfaces with all $\Gamma_{Y_{i}}$ non-degenerate. These are given by the triples

$$
\begin{aligned}
& (0,1,-1),(0,1,2),(0,1,-2),(0,2,1),(0,2,-2),(0,-1,2), \\
& (0,-1,1),(0,-2,2),(1,2,-1),(1,2,-2),(1,-1,2),(1,-2,2),
\end{aligned}
$$

surfaces $(x, y, y)$ with $x \in\{1,2\}$ and $y \in\{-2,-1,0,1,2\} \backslash\{x\}$, the surfaces $(0,1,1)$ and $(0,2,2)$ and surfaces $(x, x, x)$ with $x \in\{-2,-1,0,1,2\}$. These are 27 surfaces.

(ii) Surfaces with one $\Gamma_{Y_{i}}$ degenerate: triples $(3, y,-3)$ with $0 \leq y \leq 2$ and triples $(x, y, z)$ with $x, y \in$ $\{-2, \ldots, 2\}, z \in\{y-6, \ldots,-3\}$. These are 103 surfaces.

(iii) Surfaces with two $\Gamma_{Y_{i}}$ degenerate. These are given by the sets

$$
\begin{aligned}
& K=\{(x,-3,-3) \mid 3 \leq x \leq 9\} \\
& M(0)=\{(x, 0, z) \mid-3 \geq x \geq-6,6 \geq z \geq 3\} \\
& M(-1)=\{(x,-1, z) \mid-3 \geq x \geq-7,5 \geq z \geq 3\} \\
& M(-2)=\{(x,-2, z) \mid-3 \geq x \geq-8,4 \geq z \geq 3\} \\
& N(-2)=\{(x, y,-2) \mid-3 \geq y \geq-8,-3 \geq x \geq y-6\} \\
& N(-1)=\{(x, y,-1) \mid-3 \geq y \geq-7,-3 \geq x \geq y-6\} \\
& N(0)=\{(x, y, 0) \mid:-3 \geq y \geq-6,-3 \geq x \geq y-6\} \\
& N(1)=\{(x, y, 1) \mid-3 \geq y \geq-5,-3 \geq x \geq y-6\} \\
& N(2)=\{(x, y, 2) \mid-3 \geq y \geq-4,-3 \geq x \geq y-6\} .
\end{aligned}
$$

Adding up, these are $7+16+15+12+57+45+34+24+15=225$ surfaces.

Proof. Again, by Proposition 5.2, the theorem covers all cases, as at least one curve structure has to be non-degenerate. We consider the competely non-degenerate case (i) first. Non-degeneracy of all curve structures implies $-2 \leq n_{i} \leq 2$ for all $i$, by Corollary 4.21 .

This defines a set of 125 candidate triples $\left(x_{1}, x_{2}, x_{3}\right)$. There are 5 triples $(x, x, x)$ that only appear once, all other triples appear with multiplicity 3 via the shift relation above. Hence there are at most 45 distinct 
surfaces. These include the triples $(x, x, x)$, and 20 triples of the form $(x, y, y)$ with with $x \in\{-2,-1,0,1,2\}$ and $y \in\{-2,-1,0,1,2\} \backslash\{x\}$. Here $(x, y, y)$ is equivalent to $(-x,-y,-y)$ modulo a shift and an involution, so the distinct surfaces in this subset are given by the conditions $x \in\{1,2\}$ and $y \in\{-2,-1,0,1,2\} \backslash\{x\}$ and the surfaces $(0,1,1)$ and $(0,2,2)$. This gives 12 non-equivalent triples. Having enumerated these cases, there remain 20 triples to discuss. These can be recovered by applying the involution to the list of triples $(x, y, z)$ in the statement of the proposition that have pairwise distinct entries. By Lemma 7.11, the triples with pairwise distinct entries define non-isomorphic surfaces. This finishes the completely non-degenerate case.

We turn to the case (ii) of precisely one degenerate curve structure. Let the component of $Y$ with tamely degenerate curve structure be denoted by $Y_{1}$. By the degeneracy assumption, after maybe applying an involution, it follows from Proposition 4.19 that we can assume $n_{3} \leq-3$ and $n_{1} \leq 3$. Assume first that both conditions hold sharp, i.e. $n_{1}=3, n_{3}=-3$. From the non-degeneracy assumption on $Y_{2}$ and $Y_{3}$ it follows from Corollary 4.21 that $-2 \leq n_{2} \leq 2$ and any such choice gives a projective surface $Y$. The surfaces defined by $\left(3, n_{2},-3\right)$ are equivalent to surfaces defined by $\left(3,-n_{2},-3\right)$, so the subset $0 \leq n_{2} \leq 2$ already gives all equivalence classes. Now assume $n_{3} \leq-3$ and $n_{1}<3$. It follows from non-degeneracy of $Y_{2}$ that $-2 \leq n_{1} \leq 2$ and then one obtains $n_{1}-6 \leq n_{3} \leq-3$ by counting the total number of curves in the curve structures of the $Y_{i}$. Together with the condition $-2 \leq n_{2} \leq 2$, we obtain the set $M$ of triples $(x, y, z)$ with $x, y \in\{-2, \ldots, 2\}, z \in\{y-6, \ldots,-3\}$. None of the triples in $M$ are equivalent: suppose $(x, y, z) \sim\left(x^{\prime}, y^{\prime}, z^{\prime}\right)$, say under a sequence $T$ of shifts and involutions: assume we have $T=\imath^{a} \circ s^{b}$. Then $|z|>|x|,|y|$ shows $b=0$ and $z<0$ then shows $a=0$. The case $T=s^{a} \circ \imath^{b}$ is done the same way.

Now consider case (iii), i.e. assume that two of the curve structures are degenerate. Let $Y_{1}$ be the component with $\Gamma_{Y_{1}}$ non-degenerate, and $Y_{2}, Y_{3}$ be the components with tamely degenerate curve structures. As above, by Proposition 4.19, degeneracy on $Y_{2}$ implies $n_{1} \leq-3$ or $n_{2} \geq 3$ and from $Y_{3}$ we get $n_{2} \leq-3$ or $n_{3} \geq 3$. Suppose first $n_{1} \leq-3$ and $n_{2} \geq 3$. Then $n_{1}=-3$ and $n_{2}=3$ by counting curves on $Y_{2}$. It follows that $3 \leq n_{3} \leq 9$, giving the set $K$ of triples $(x,-3,3)$ with $3 \leq x \leq 9$. If $n_{2} \leq-3$ and $n_{3} \geq 3$ one obtains equivalent tupels thus isomorphic surfaces.

Now assume $n_{1} \leq-3$ and $n_{2} \leq 2$. By counting curves, we have

$$
\begin{aligned}
-3 & \geq n_{1} \geq n_{2}-6 \\
2 & \geq n_{2} \geq n_{3}-6 \\
-2 & \leq n_{3} \leq 6+n_{2} .
\end{aligned}
$$

We shall first consider the case $n_{3} \geq 3$. Then $2 \geq n_{2} \geq-3$. This gives the following sets:

$$
\begin{aligned}
& M(2)=\{(x, 2, z) \mid-3 \geq x \geq-4,8 \geq z \geq 3\} \\
& M(1)=\{(x, 1, z) \mid-3 \geq x \geq-5,7 \geq z \geq 3\} \\
& M(0)=\{(x, 0, z) \mid-3 \geq x \geq-6,6 \geq z \geq 3\} \\
& M(-1)=\{(x,-1, z) \mid-3 \geq x \geq-7,5 \geq z \geq 3\} \\
& M(-2)=\{(x,-2, z) \mid-3 \geq x \geq-8,4 \geq z \geq 3\} \\
& M(-3)=\{(x,-3,3) \mid-3 \geq x \geq-9\}
\end{aligned}
$$

Applying the involution and a shift we see that the sets $M(i)$ and $M(-i)$ describe equivalent triples and hence the same isomorphism classes of surfaces for $i=1,2$, as do $M(-3)$ and $M_{0}$. 
Now suppose again $n_{1} \leq-3$ and $n_{2} \leq 2$, but assume $n_{3} \leq 2$, so necessarily $n_{2} \leq-3$. Then by nondegeneracy of $Y_{1}$ we must have $2 \geq n_{3} \geq-2$. We obtain the following sets:

$$
\begin{aligned}
& N(-2)=\{(x, y,-2) \mid-3 \geq y \geq-8,-3 \geq x \geq y-6\} \\
& N(-1)=\{(x, y,-1) \mid-3 \geq y \geq-7,-3 \geq x \geq y-6\} \\
& N(0)=\{(x, y, 0) \mid-3 \geq y \geq-6,-3 \geq x \geq y-6\} \\
& N(1)=\{(x, y, 1) \mid-3 \geq y \geq-5,-3 \geq x \geq y-6\} \\
& N(2)=\{(x, y, 2) \mid-3 \geq y \geq-4,-3 \geq x \geq y-6\} .
\end{aligned}
$$

We observe that the sets $M(i)$ and the $N(i)$ contain non-equivalent triples: this follows, as the number $i$ only appears as an entry in $M(i)$ and then $x, y$ have to have alternating signs, so $(x, y, i)$ is not contained in any of the $N(i)$. This shows that models defined by the $N(i)$ are not isomorphic to models defined by triples in the sets $M(i)$. The set $N(i)$ also parameterises models not isomorphic to any model in any $N(k)$ if $i \neq k$ : let $(x, y, i) \in N(i)$. Assume there is $\left(x^{\prime}, y^{\prime}, i^{\prime}\right) \in N\left(i^{\prime}\right)$ with $(x, y, i) \sim\left(x^{\prime}, y^{\prime}, i^{\prime}\right), i^{\prime} \neq i$, i.e. there exists a composition $T$ of shifts and involutions with $T(x, y, i)=\left(x^{\prime}, y^{\prime}, i^{\prime}\right)$. Since the absolute value of the middle entry of these triples is smaller than the absolute value of the other entries, this is only possible if $i^{\prime}=-i$ and $T=1$. But now the claim follows from the fact that the third entry is always negative. Thus, the $N(i)$ parameterize distinct surfaces. The remaining cases, $n_{2} \geq 3, n_{1} \geq-2$ and $n_{3} \geq 3$ or $n_{3} \leq 2$ desribe the same models: $(x, y, z) \sim(z, x, y) \sim(-x,-z,-y)=:\left(x^{\prime}, y^{\prime}, z^{\prime}\right)$, and thus from $x \leq-3, y \leq 2$, it follows $x^{\prime} \geq 3, z^{\prime} \leq-2$. Then $z \geq 3$ gives $y^{\prime} \leq-3$ and $z \leq 2$ gives $y^{\prime} \geq-2$. This concludes the proof.

This concludes the count: if $Y \in \operatorname{PMod}(\mathscr{P})$, then either all curve structures of components of $Y$ are regular or at least one of the curve structures is very degenerate. In both cases, there are either one, two or three components of $Y$ with non-degenerate curve structure. The cases where all curve structures are regular are covered in Theorem 7.12, while the cases with at least one very degenerate curve structure are covered in Theorem 7.8. Summarizing these results we obtain:

Theorem 7.13. There are $457=104+353$ surfaces in $\operatorname{PMod}_{2}(\mathscr{P})$.

We point out that the number of these surfaces is the number of orbits of maximal cones of the Mori fan under action of the birational group.

7.3.1. Mori fan.- We can now count the number of maximal cones of the Mori fan of the DNV family of degree 2. For this we will have to take the action of the birational automorphism group and its action on the maximal cones of the Mori fan into account, which we discussed at the end of Section 6. We will first count the number of symmetric models.

Proposition 7.14. There are 22 symmetric models of the DNV family of degree 2. More precisely, the symmetric models $\mathcal{Y}$ of the DNV family of degree 2 of class $\mathscr{P}$ are

(i) the models $\{(0, n,-n) \mid n \in\{-3, \ldots 6\}\}$, with notation as in Section 7.3 and

(ii) the model such that

(a) $\Gamma_{Y_{i}}$ is very degenerate for $i=1,3$ and

(b) the intersection numbers are $D_{12}^{2}=D_{32}^{2}=4, D_{13}^{2}=D_{31}^{2}=-1, D_{21}^{2}=D_{23}^{2}=-6$.

The symmetric models of class $\mathscr{T}$ are given by $(x, x)$ with $x \in\left\{0,-1,-2,-3, \ldots,-8,-8^{\prime},-9\right\}$, with notation as in the proof of Theorem 7.7.

Proof. We first count symmetric models of class $\mathscr{P}$. A model $\mathcal{Y}$ is symmetric if and only if there is an automorphism of $\mathcal{Y}_{c}$ that identifies two of the components, say $Y_{1}$ and $Y_{3}$. We have mentioned that this implies $D_{13}^{2}=D_{31}^{2}=-1$. As $Y_{1} \cong Y_{3}$, our model is thus completely specified by the curve sructure of $Y_{1}$. The curve structure $\Gamma_{Y_{1}}$ is obtained from $\bigcup_{2}$. The condition $D_{13}^{2}=-1$ implies that in fact, $Y_{1}$ is obtained from blow-ups or blow-downs in the interior special point $p \in D_{12}$. If $\Gamma_{Y_{1}}$ is regular, it follows that, in the 
teminology of Section 7.3, $n_{1} \leq 6$. Because of the symmetry, $D_{12}^{2}=D_{32}^{2}$ and hence $n_{1} \geq-3$. Conversly, each such choice of $n_{1}$ implies a unique symmetric model. If $\Gamma_{Y_{1}}$ is very degenerate, then the model is uniquely determined, compare Proposition 6.41: we have $D_{12}^{2}=D_{32}^{2}=4, D_{13}^{2}=D_{31}^{2}=-1, D_{21}^{2}=D_{23}^{2}=-6$ and also $\Gamma_{Y_{3}}$ is very degenerate. Also, both $\Gamma_{Y_{1}}$ and $\Gamma_{Y_{3}}$ have three vertices while $\Gamma_{Y_{2}}$ has 18 vertices.

For $\mathcal{Y}_{c} \in \operatorname{PMod}_{2}(\mathscr{T})$, being symmetric is the same as having isomorphic smooth components: indeed, suppose $Y_{1}, Y_{3}$ are the smooth components, $Y_{\omega}$ is the special component and suppose there is an isomorphism $\gamma: Y_{1} \rightarrow Y_{3}$. By Proposition 7.3, we can assume $\gamma$ maps $Y_{1} \cap Y_{\omega}$ to $Y_{2} \cap Y_{\omega}$. Then one can show - using the morphism $\bar{\psi}$ defined in Section 7.1 - that there is an automorphism $\psi_{\omega}$ on $Y_{\omega}$ that exchanges the nodal components of the anticanonical cylce of $Y_{\omega}$. Using $\psi_{\omega}$ and $\gamma$ (and maybe an involution on the smooth components), from the universal property of pushouts, one gets an automorphism of $\mathcal{Y}_{c}$ that maps $Y_{1}$ to $Y_{3}$. A similar reasoning as above gives the set of symmetric models of class $\mathscr{T}$. Alternatively, it is easy to see that these are precisely the models that can be obtained by a type II flop from a symmetric model of class $\mathscr{P}$.

We can now count all maximal cones of the Mori fan.

Theorem 7.15. Let $\mathcal{Y} \rightarrow S$ be a model of the Dolgachev-Nikulin-Voisin family. Then $\operatorname{MF}(\mathcal{Y} / S)$ has 3460 maximal cones. Of these 753 are associated to a model of class $\mathscr{T}$ and 2707 are associated to a model of class $\mathscr{P}$.

Proof. By Proposition 7.14, there are 11 symmetric models in $\operatorname{PMod}(\mathscr{T})$, out of 131 isomorphism classes of models of class $\mathscr{T}$ in total (Theorem 7.7). It now follows from Proposition 6.43 that there are 120 models having 6 associated cones and 11 models having 3 associated cones, giving us $120 \times 6+11 \times 3=753$ maximal cones.

Again by Proposition 7.14, there are 11 symmetric models $\operatorname{PMod}(\mathscr{P})$. By Theorem 7.13, there are 457 projective models of class $\mathscr{P}$ in total, hence, by Proposition 6.43, there are $457-11=446$ models having 6 associated cones, 10 models having 3 associated cones and the model $\mathcal{Y}_{\mathscr{P}}$ defining a unique cone in the Mori fan. This defines $446 \times 6+10 \times 3+1=2707$ maximal cones. Altogether, we obtain $753+2707=3460$ maximal cones.

\section{The Secondary fan}

In this section, as an application of our results, we give a description of the secondary fan of the DNV family of degree 2, as introduced in [HKY20]. This fan is obtained by coarsening the Mori fan of $\mathcal{Y}$. Roughly, it is obtained by deleting all facets that correspond to flops that do not change the dual intersection graph.

\subsection{Preliminaries}

For a cone $C$ in a vector space we denote by $\operatorname{Int} C$ its interior and by Relint $C$ the relative interior of $C$. Let $\mathcal{Y} \rightarrow S$ be a model of the DNV family of degree 2. The set of maximal cones of $M F(\mathcal{Y} / S)$ will be denoted by $\operatorname{MF}_{\max }(\mathcal{Y} / S)$. We recall that $\operatorname{MF}(\mathcal{Y} / S)$ contains only finitely many cones and that these are all rational polyhedral.

Recall that any interior facet $\tau$ of $\operatorname{MF}(\mathcal{Y} / S)$ corresponds to a flop $f_{\tau}: \mathcal{Y}^{\prime} \rightarrow \mathcal{Y}^{\prime \prime}$ for $\mathcal{Y}^{\prime}, \mathcal{Y}^{\prime \prime}$ models of the DNV family, see Proposition 3.9. By Corollary $6.12, f_{\tau}$ is a type I or type II flop and we will correspondingly call $\tau$ of type I or type II. We denote by $\mathcal{F}$ the set of all interior facets that correspond to type II flops and set $\mathcal{M}=|\operatorname{MF}(\mathcal{Y} / S)| \backslash \cup_{\tau \in \mathcal{F}}|\tau|$. Let $\mathcal{C}$ be a connected component of $\mathcal{M}$ and let $C(f)$ be a cone of $\operatorname{MF}(\mathcal{Y} / S)$ such that Int $C(f) \cap \mathcal{C} \neq \varnothing$, defined by a marked model $\left(\mathcal{Y}_{f}, f\right)$, i.e. a map $f: \mathcal{Y} \rightarrow \mathcal{Y}_{f}$. Let $I(f)$ be the set of all maximal cones of $\operatorname{MF}(\mathcal{Y} / S)$ consisting of cones $C(g)$ corresponding to models $\left(\mathcal{Y}_{g}, g\right)$, such that there 
is a sequence of type I flops $\phi: \mathcal{Y}_{f} \rightarrow \mathcal{Y}^{\prime}$ and an isomorphism $\gamma: \mathcal{Y}^{\prime} \rightarrow \mathcal{Y}_{g}$ giving a commutative diagram

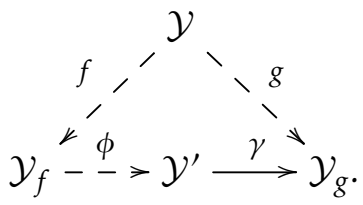

By the construction of $\mathcal{M}$ and $\mathcal{C}$ we have $\mathcal{C} \subset \cup_{g \in I(f)} C(g)$ and in fact

$$
\overline{\mathcal{C}}=\cup_{g \in I(f)} C(g) \text {. }
$$

It is immediate that this construction does not depend on the choice of $\mathcal{Y} \rightarrow S$ in the sense that if $\mathcal{Y}^{\prime} \rightarrow S$ is another model and $\phi: \mathcal{Y} \rightarrow \mathcal{Y}^{\prime}$ is a birational isomorphism, the identification $\phi_{*} \operatorname{MF}(\mathcal{Y} / S) \cong \operatorname{MF}\left(\mathcal{Y}^{\prime} / S\right)$ is compatible with the construction of $\mathcal{M}$.

It is known that the closures of the connected components of $\mathcal{M}$ define a fan, the secondary fan $\mathrm{MF}^{2 \mathrm{nd}}(\mathcal{Y} / S)$ of $\mathcal{Y}$, as can be shown by adapting the techniques in [HKY20]. However, as no published proof is available, we offer an alternative proof of this fact via the results of this paper.

By Proposition 6.43, there is a unique cone $\sigma_{\mathscr{P}}$ associated to $\mathcal{Y}_{\mathscr{P}}$. Write $\mathcal{C}_{\mathscr{P}}$ for the connected component of $\mathcal{M}$ such that $\mathcal{C} \cap$ Int $\sigma_{\mathscr{P}} \neq \varnothing$.

Lemma 8.1. Let $\mathcal{C}$ be a connected component of $\mathcal{M}$. Let $\overline{\mathcal{C}}=\cup_{g \in I(f)} C(g)$, with maps $g: \mathcal{Y} \rightarrow \mathcal{Y}_{g}$. Then the following holds:

(i) If $\mathcal{C}=\mathcal{C}_{\mathscr{P}}$, then $\left(\mathcal{Y}_{g}\right)_{c} \in \operatorname{PMod}_{2}(\mathscr{P})$ for all $g$.

(ii) If $\mathcal{C} \neq \mathcal{C}_{\mathscr{P}}$, then $\left(\mathcal{Y}_{g}\right)_{c} \in \operatorname{PMod}_{2}(\mathscr{T})$ for all $g$.

Proof. This follows from the definition of $\mathcal{M}$, Lemma 6.14 and Proposition 6.43 : there is only one cone corresponding to $\mathcal{Y}_{\mathscr{P}}$ and any model $\mathcal{Y}^{\prime}$ of class $\mathscr{P}$ can be obtained from $\mathcal{Y}_{\mathscr{P}}$ by a sequence of type I flops.

We will call the components of $\mathcal{M}$ other than $\mathcal{C}_{\mathscr{P}}$ components of type $\mathscr{T}$. We now calculate the number of connected components of $\mathcal{M}$. We first prove some lemmas on sequences of flops. Recall that a model $\mathcal{Y}$ is symmetric if there is an automorphism of the special fibre $\mathcal{Y}_{c}$ that identifies two components of $\mathcal{Y}_{c}, c f$. Definition 6.40 .

Lemma 8.2. Let $\mathcal{Y}$ be a model with $\mathcal{Y}_{c} \in \operatorname{PMod}_{2}(\mathscr{T})$. Assume $\mathcal{Y}$ is symmetric. Let $F \in \operatorname{Mov}(\mathcal{Y} / S)$ be a divisor and suppose

$$
\mathcal{Y} \rightarrow \mathcal{Y}_{1} \rightarrow \cdots \rightarrow \mathcal{Y}_{n} \rightarrow \mathcal{Y}
$$

is a sequence of F-flops. Then at least one flop in this sequence is of type II.

Proof. We show that the assumption that all flops are of type I leads to a contradiction. Consider the model $\mathcal{Y}_{\mathscr{P}}$. By Proposition 6.8, any elementary modification of type II of $Y_{\mathscr{P}}$ lifts to a type II flop on $\mathcal{Y}_{\mathscr{P}}$. Let $\mathcal{Y}_{I}$ be the model obtained by applying a single type II flop $\phi$ to $\mathcal{Y}_{\mathscr{P}}$. Then $\mathcal{Y}_{I}$ is symmetric. By Corollary 6.32, there is a composition of type I flops $\mathcal{Y}_{\mathscr{T}} \rightarrow \mathcal{Y}_{I}$ and $\mathcal{Y}_{\mathscr{T}} \rightarrow \mathcal{Y}$. Thus there is a composition of maps

$$
\mathcal{Y}_{I} \rightarrow \mathcal{Y}_{\mathscr{T}} \rightarrow \mathcal{Y} \rightarrow \mathcal{Y} \rightarrow \mathcal{Y}_{\mathscr{T}} \rightarrow \mathcal{Y}_{I}
$$

that factors into a sequence of $H$-flops of type I for some $H \in \operatorname{Mov}\left(\mathcal{Y}_{I} / S\right)$. Hence it is enough to show the Lemma for $\mathcal{Y}=\mathcal{Y}_{I}$. In this case, by applying a type II flop $\mathcal{Y}_{I} \rightarrow \mathcal{Y}_{\mathscr{P}}$, Sequence 8.2 induces a sequence

$$
\mathcal{Y}_{\mathscr{P}} \rightarrow \mathcal{Y}_{1}^{\prime} \rightarrow \cdots \rightarrow \mathcal{Y}_{n}^{\prime} \rightarrow \mathcal{Y}_{\mathscr{P}}
$$

of $\left(\phi^{-1}\right)_{*} F$-flops, as $\phi$ contracts a curve that is disjoint from all the exceptional loci. From Corollary 6.38 it follows that $n=0$. Hence a sequence as (8.2) does not exist. 
Lemma 8.3. Let $\mathcal{Y}$ be a model of the DNV family of degree 2 with $\mathcal{Y}_{c} \in \operatorname{PMod}_{2}(\mathscr{T})$. Assume $\mathcal{Y}$ is not symmetric. Then there is a sequence of type I flops

$$
\mathcal{Y} \rightarrow \mathcal{Y}_{1} \rightarrow \cdots \rightarrow \mathcal{Y}_{n} \rightarrow \mathcal{Y}
$$

such that the composition $\gamma$ of these flops is not an automorphism.

Proof. Write $\mathcal{Y}_{c}=Y_{1} \cup Y_{2} \cup Y_{3}$ with $Y_{1}$ the special component. As $\mathcal{Y}$ is not symmetric, $Y_{2}$ and $Y_{3}$ are not isomorphic. Given $\mathcal{Y}$, by Proposition 6.32, there exists a sequence $\phi: \mathcal{Y} \rightarrow \mathcal{Y}_{\mathscr{T}}$ of type I flops. Necessarily, $\phi$ maps the special component to the special component. Let $\psi$ be the automorphism from Proposition 6.45. Consider the composition $\gamma=\phi^{-1} \circ \psi \circ \phi: \mathcal{Y} \rightarrow \mathcal{Y}$. By construction, $\gamma$ maps $Y_{2}$ to $Y_{3}$ and $Y_{3}$ to $Y_{2}$. As these components are not isomorphic, it follows that $\gamma$ is not an automorphism. Being a composition of small modifications, $\gamma$ has a factorisation

$$
\mathcal{Y} \rightarrow \mathcal{Y}_{1} \rightarrow \cdots \rightarrow \mathcal{Y}_{n} \rightarrow \mathcal{Y}
$$

into $F$-flops for a divisor $F \in \operatorname{Mov}(\mathcal{Y} / S)$. Note that the smooth component $D_{\omega}$ of the restriction of the double curve of $\mathcal{Y}$ to $Y_{1}$ is disjoint from $\operatorname{Ex}(\gamma)$. Hence, by Corollary 6.12, all flops in the factorisation (8.3) are of type I and we thus obtain the desired sequence.

Proposition 8.4. The topological space $\mathcal{M}$ has 4 connected components. These are given by $\mathcal{C}_{\mathscr{P}}$ and 3 components of type $\mathscr{T}$.

Proof. Here we chose $\mathcal{Y}_{\mathscr{T}}$ as a reference model and hence consider $\operatorname{MF}\left(\mathcal{Y}_{\mathscr{T}} / S\right)$. By Proposition 6.43, there are 3 cones in the orbit of $\operatorname{Nef}\left(\mathcal{Y}_{\mathscr{T}} / S\right)$. We can write these as $C\left(f_{1}\right), C\left(f_{2}\right), C\left(f_{3}\right)$ where $f_{1}=\mathrm{id}_{\mathcal{Y}_{\mathscr{T}}}$ and $f_{2}, f_{3} \in \operatorname{Bir}\left(\mathcal{Y}_{\mathscr{T}} / S\right)$ are birational automorphisms of $\mathcal{Y}_{\mathscr{T}} \rightarrow S$. Let $\mathcal{C}_{i}$ denote the connected component of $\mathcal{M}$ such that $C\left(f_{i}\right) \subset \overline{\mathcal{C}}_{i}$. By Lemma 8.2 applied to $\mathcal{Y}_{\mathscr{T}}$, the $\mathcal{C}_{i}$ are all distinct. Let $\sigma$ be a cone associated to a model $\mathcal{Y}^{\prime}$ of class $\mathscr{T}$. We will show that there is an $i \in\{1,2,3\}$ such that $\sigma \subset \overline{\mathcal{C}}_{i}$. This implies the result, as all cones such that the associated model has class $\mathscr{P}$ are contained in the closure of $\mathcal{C}_{\mathscr{P}}$. By Corollary 6.32, there is a composition of type I flops $f: \mathcal{Y}_{\mathscr{T}} \rightarrow \mathcal{Y}^{\prime}$. This defines cones $C\left(f \circ f_{i}\right), i=1, \ldots, 3$ with associated model $\mathcal{Y}^{\prime}$. The cone $C\left(f \circ f_{i}\right)$ is contained in $\overline{\mathcal{C}}_{i}$ by construction. So we are done if $\mathcal{Y}^{\prime}$ is symmetric, as there are then precisely 3 cones with associated model $\mathcal{Y}^{\prime}$, by Proposition 6.43 .

Suppose $\mathcal{Y}^{\prime}$ is not symmetric. By Proposition 6.43 , there are 6 cones associated to $\mathcal{Y}^{\prime}$. We shall show that each of these cones lies in some $\overline{\mathcal{C}}_{i}$. Since $\mathcal{Y}^{\prime}$ is not symmetric, it follows from Lemma 8.3 that there is a non-trivial sequence of type I flops $\gamma: \mathcal{Y}^{\prime} \rightarrow \mathcal{Y}^{\prime}$. Then $C\left(\gamma \circ f \circ f_{i}\right)$ is not equal to $C\left(f \circ f_{i}\right)$, as $\gamma$ is not an automorphism and $C\left(\gamma \circ f \circ f_{i}\right) \subset \overline{\mathcal{C}}_{i}$ by definition of $\mathcal{C}_{i}$. This shows that all cones associated to $\mathcal{Y}^{\prime}$ are contained in some $\overline{\mathcal{C}}_{i}$.

\subsection{Flopping along a line}

Recall that we are, by Theorem 3.4, in a Mori Dream space situation (in degree 2). The following is then a standard construction: let $F$ be a $\mathbb{Q}$-divisor in $\operatorname{Mov}(\mathcal{Y} / S)$. Suppose $F$ is not nef on $\mathcal{Y}$ and there is a cone $\sigma_{F} \in \operatorname{MF}_{\max }(\mathcal{Y} / S)$ with $F \in \operatorname{Int} \sigma_{F}$. Let $A$ be an ample divisor and define $L=L(F, A)$ to be the line segment connecting $F$ and $A$. Since $L$ is spanned by interior points of a convex cone it is itself contained in the interior. Suppose that for any facet $\tau \in \operatorname{MF}(\mathcal{Y} / S), L \cap \tau \neq \varnothing$ implies $L \cap \tau \subset \operatorname{Relint}(\tau)$. This means that the line $L$ intersects maximal cones and their facets as nicely as possible. Note that (by convexity), this implies that if $\sigma \in \operatorname{MF}_{\max }(\mathcal{Y} / S)$, at most two facets of a cone $\sigma$ are met, and the only maximal cones $\sigma$ such that there is a unique facet $\tau \subset \sigma$ meeting $L$ are $\sigma_{F}$ and $\sigma_{A}=\operatorname{Nef}(\mathcal{Y} / S)$. Let $\left\{\gamma_{i}\right\}_{i}$ be the collection of maximal cones of $\operatorname{MF}(\mathcal{Y} / S)$ such that $L \cap \operatorname{Int} \gamma_{i} \neq 0$. Note that this collection is finite by Theorem 3.4(i). Denote the unique facet of $\sigma_{A}$ met by $L$ by $\tau$ and let $R$ be the extremal ray corresponding to $\tau$. Then $F$ is stricly negative on $R$. Consider the contraction morphism $\operatorname{contr}_{R}: \mathcal{Y} \rightarrow \mathcal{Z}$. It is a small contraction as $\tau$ is interior and hence defines an $F$-flop $\phi: \mathcal{Y} \rightarrow \mathcal{Y}^{+}$. This gives a canonical linear isomorphism $\phi_{*}: \mathrm{N}^{1}(\mathcal{Y} / S) \rightarrow \mathrm{N}^{1}\left(\mathcal{Y}^{+} / S\right)$. 
Choose an ample divisor $A^{+} \in \operatorname{Pic}\left(\mathcal{Y}^{+} / S\right)$ on $\phi_{*}(L)$. Now we can consider the divisor $\phi_{*} F$ and the line segment $L\left(\phi_{*} F, A^{+}\right)$and repeat the argument for this data. In this way we obtain a finite sequence of $F$-flops

$$
\mathcal{Y} \rightarrow \mathcal{Y}_{1} \rightarrow \cdots \rightarrow \mathcal{Y}_{n}
$$

We will call this sequence a sequence of F-flops induced by $L$. We note that this may depend on the choice of $A$ and hence $L$, but not on the choice of $A^{+}$and subsequent choices. The truncations $\phi_{l}: \mathcal{Y} \rightarrow \mathcal{Y}_{l}$ define maximal cones of $\operatorname{MF}(\mathcal{Y} / S)$ and by construction, for each $\gamma_{i}$ there is an $l$ such that $\gamma_{i}=\phi_{l}^{*}\left(\operatorname{Nef}\left(\mathcal{Y}_{l} / S\right)\right)$. Note also that $\gamma_{n}=\sigma_{F}$ by construction.

Definition 8.5. Let $\mathcal{C}$ be a connected component of $\mathcal{M}$ with closure $\overline{\mathcal{C}}$. A $\mathcal{C}$-test segment is a line segment $L(p, q)$ such that $p, q$ are points in $\mathcal{C}$, such that there are cones $\sigma_{p}, \sigma_{q} \in \mathrm{MF}_{\max }(\mathcal{Y} / S)$ with $p \in \operatorname{Int}\left(\sigma_{p}\right)$ and $q \in \operatorname{Int}\left(\sigma_{q}\right)$. A test segment $L$ is called nice if for any interior facet $\tau \in \operatorname{MF}(\mathcal{Y} / S), L \cap \tau \neq \varnothing$ implies $L \cap \tau \subset \operatorname{Relint}(\tau)$.

Given a test segment $L(p, q)$, we assume for simplicity that $\sigma_{q}=\operatorname{Nef}(\mathcal{Y} / S)$. By choosing a different model, which does not change the geometry of the Mori fan, we can always assume that we are in this situation.

We now fix a connected component $\mathcal{C}$ of $\mathcal{M}$ with closure $\overline{\mathcal{C}}$. Let $L=L(p, q)$ be a line segment. We define

$$
\begin{gathered}
\mathrm{M}(L)=\left\{\sigma \in \mathrm{MF}_{\max }(\mathcal{Y} / S) \mid L \cap \sigma \neq \varnothing\right\}, \\
\mathrm{I}(L)=\{\sigma \in \mathrm{M}(L) \mid L \cap \operatorname{Int}(\sigma) \neq \varnothing\} .
\end{gathered}
$$

For a cone $\sigma$ we write $F(\sigma)$ for the set of subcones of the Mori fan of codimension 1 that are contained in $\sigma$, i.e. the facets of $\sigma$. We set

$$
\mathrm{N}(L)=\{\sigma \in \mathrm{I}(L) \mid \forall \tau \in F(\sigma), L \cap \tau \subset \operatorname{Relint}(\tau)\}
$$

Note that the conditions to be in $I(L)$ and $N(L)$ are open conditions, so if $L^{\prime}$ is a line segment contained in a small cylinder containing $L$, then $\mathrm{I}(L) \subset \mathrm{I}\left(L^{\prime}\right)$ and $\mathrm{N}(L) \subset \mathrm{N}\left(L^{\prime}\right)$. Also note that if $L$ is a nice test segment, $\mathrm{M}(L)=\mathrm{I}(L)=\mathrm{N}(L)$.

Proposition 8.6. Let $\overline{\mathcal{C}}$ be the closure of the connected component $\mathcal{C}$ of $\mathcal{M}$. Let $L=L(p, q)$ be a nice $\mathcal{C}$-test segment where both $p$ and $q$ are divisors (with integral coefficients). Let

$$
\phi_{L}: \mathcal{Y} \rightarrow \mathcal{Y}_{1} \rightarrow \cdots \rightarrow \mathcal{Y}_{n}
$$

be the sequence of flops induced by $L$. Then $\gamma \in \mathrm{M}(L)$ implies $\gamma \subset \overline{\mathcal{C}}$.

Proof. Let $\sigma_{p}$ and $\sigma_{q}$ be the cones containing $p$ and $q$, respectively. We assume $\sigma_{q}=\operatorname{Nef}(\mathcal{Y} / S)$. By Lemma 8.1, there are two cases: either $\mathcal{C}=\mathcal{C}_{\mathscr{P}}$ or the two models corresponding to $\sigma_{p}$ and $\sigma_{q}$ are of type $\mathscr{T}$.

We first consider $\mathcal{C}=\mathcal{C}_{\mathscr{P}}$. Then, as $L$ is a nice test segment, it follows from the definition of $\mathcal{C}$ that $\mathcal{Y}$ and $\mathcal{Y}_{n}$ have dual intersection complex $\mathscr{P}$. Then, by Lemma 6.14, all flops in the sequence $\phi_{L}$ are of type I and thus all $\mathcal{Y}_{i}$ have dual intersection complex $\mathscr{P}$. Hence all cones associated to $\mathcal{Y}_{i}$ are in $\overline{\mathcal{C}}$, implying the claim.

Now consider the only other possible case, namely that the two models corresponding to $\sigma_{p}$ and $\sigma_{q}$ are of type $\mathscr{T}$. Then $\mathcal{Y}_{\sigma_{p}}$ and $\mathcal{Y}$ have dual intersection complex $\mathscr{T}$. By construction of $\mathcal{C}$ we have a map $\psi: \mathcal{Y} \rightarrow \mathcal{Y}_{\sigma_{p}}$ that is given by a series of type I flops. Note that these are not necessarily $F$-flops for a divisor $F \in \operatorname{Mov}(\mathcal{Y} / S)$. However, the map $\psi$ can also be written as a sequence

$$
\mathcal{Y} \rightarrow \mathcal{Y}_{1}^{\prime} \rightarrow \cdots \rightarrow \mathcal{Y}_{\sigma_{p}}
$$


of $F$-flops for some divisor $F \in \operatorname{Mov}(\mathcal{Y} / S)$. Any flop in this sequence is necessarily of type I. By definition of the Mori fan, we have an isomorphism $\pi: \mathcal{Y}_{\sigma_{p}} \cong \mathcal{Y}_{n}$ giving rise to a commutative diagram

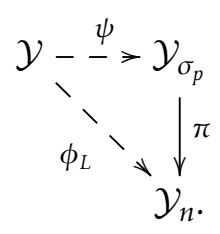

As $\psi$ is a composition of type I flops, it maps smooth components of $\mathcal{Y}_{c}$ to smooth components and the same is true for $\pi$. Hence $\phi_{L}$ also maps the special component $\left(\mathcal{Y}_{c}\right)_{\omega}$ to the special component $\left(\left(\mathcal{Y}_{n}\right)_{c}\right)_{\omega}$. We claim that this implies that all flops in the factorisation of $\phi$ are of type I. To show the claim, we assume there is a type II flop in the sequence and derive a contradiction. Let

$$
\mathcal{Y}_{k} \rightarrow \mathcal{Y}_{k+1} \rightarrow \cdots \rightarrow \mathcal{Y}_{n}
$$

be the tail of the sequence where $\phi_{k}: \mathcal{Y}_{k} \rightarrow \mathcal{Y}_{k+1}$ is the first type II flop. Being type II, writing $\left(\mathcal{Y}_{k+1}\right)_{c}=$ $Y_{1} \cup Y_{2} \cup Y_{3}$, the flopped curve $C$ is given by a component of the double curve, say by $D_{12}=Y_{1} \cap Y_{2}$ on $Y_{1}$ and $D_{21}$ on $Y_{2}$, with $D_{21}^{2}=D_{12}^{2}=-1$. Also, the birational transform of $\left(\mathcal{Y}_{c}\right)_{\omega}$ on $\mathcal{Y}_{k+1}$ is $Y_{3}$. Because $\mathcal{Y}_{n}$ has dual intersection complex $\mathscr{T}$, there is at least 1 more type II flop in Sequence 8.4, say $\phi_{l}$. By Lemma 6.14 this is the only further type II flop. Because $\phi$ maps $\left(\mathcal{Y}_{c}\right)_{\omega}$ to $\left(\left(\mathcal{Y}_{n}\right)_{c}\right)_{\omega}$, the exceptional locus $\operatorname{Ex}\left(\phi_{l}\right)$ is given by the birational transform $C_{l}$ of $C$ on $\mathcal{Y}_{l}$. Also, the preimage of $C_{l}$ under $v:\left(\mathcal{Y}_{l}\right)_{c}^{v} \rightarrow\left(\mathcal{Y}_{l}\right)_{c}$ consist of two $(-1)$-curves. Hence all of the flops $\phi_{s}, l-1 \geq s \geq k+1$ have exceptional loci disjoint from the birational transform $C_{s}$ of $C$ on $\mathcal{Y}_{s}$. But this implies that for the birational transform $F_{l}$ of $F$ on $\mathcal{Y}_{l}$ we have $F_{l} \cdot C_{l}>0$, as $F_{k+1} \cdot C>0$. But the contraction defining $\phi_{l}$ contracts $C_{l}$, a contradiction. Hence all flops in $\phi$ are of type I. In particular, by Equation 8.1, all maximal cones met by $L$ are in the closure of $\mathcal{C}$ and thus $\gamma \subset \overline{\mathcal{C}}$.

\subsection{Reduction}

In this section we show that it is enough to check convexity on line segments defining sequences of $F$-flops as in the previous section. The idea is as follows: if $L$ is a line segment through two points in a connected component $\mathcal{C}$, by definition of the connected components, it is enough to check that any maximal cone $\sigma$ of the Mori fan met by $L$ is contained in the closure $\overline{\mathcal{C}}$ of $\mathcal{C}$. For this, one checks that there is a nice test segment $L^{\prime}$ from some cone in $\mathcal{C}$ that meets $\sigma$, which implies that all cones met by $L^{\prime}$ are contained in $\overline{\mathcal{C}}$. Such a test segment can be found by wiggling the line segment $L$, as for one it is enough to check convexity on the interior of the connected components and moreover $\operatorname{MF}(\mathcal{Y} / S)$ has only finitely many cones. We give the details. The following lemma, which we include for completeness, is elementary.

Lemma 8.7. Let $U$ be a convex set in a normed vector space $V$. Then the closure $\bar{U}$ and the interior $U^{o}$ are convex.

Lemma 8.8. Let $\overline{\mathcal{C}}$ be the closure of a connected component of $\mathcal{M}$ and let $p_{0}, q_{0}$ be points in the interior $\overline{\mathcal{C}}^{\circ}$. Set $L_{0}=L\left(p_{0}, q_{0}\right)$ and assume $\gamma \in \mathrm{MF}_{\max }(\mathcal{Y} / S)$. If $L_{0} \cap \gamma \neq \varnothing$, then there is a $\mathcal{C}$-test segment $L$ with $\gamma \in \mathrm{N}(L)$, $\mathrm{I}\left(L_{0}\right) \subset \mathrm{I}(L)$ and $\mathrm{N}\left(L_{0}\right) \subset \mathrm{N}(L)$.

Proof. Given data as in the statement of the lemma, suppose $L_{0} \cap \gamma \neq \varnothing$. Then by translating both $p_{0}$ and $q_{0}$ by a small amount we can find points $p_{1}, q_{1} \in \mathcal{C}^{0}$ such that $L_{1}=\left(p_{1}, q_{1}\right)$ intersects $\gamma$ in its interior. If the translation is small enough we can assume that $\mathrm{I}\left(L_{0}\right) \subset \mathrm{I}\left(L_{1}\right)$ and $\mathrm{N}\left(L_{0}\right) \subset \mathrm{N}\left(L_{1}\right)$, as the relevant conditions are open and there are only finitely many cones in $\operatorname{MF}(\mathcal{Y} / S)$.

Let $\tau_{1} \in F(\gamma)$ such that $L_{1} \cap \tau_{1}=\{x\}$, with $x \notin \operatorname{Relint}\left(\tau_{1}\right)$. Let $\tau_{2}$ be a facet of $\tau_{1}$ with $x \in \tau_{2}$. Let $v$ be a vector tangent to $\tau_{1}$ but not tangent to $\tau_{2}$ to such that $x+v \in \operatorname{Relint}\left(\tau_{1}\right)$. Then by translating both $p_{1}$ and $q_{2}$ by some $\varepsilon v$ we can find points $p_{2}, q_{2} \in \mathcal{C}^{0}$ such that $L_{2}=\left(p_{2}, q_{2}\right)$ intersects $\gamma$ in its interior, $L_{2} \cap \tau_{1} \subset \operatorname{Relint}\left(\tau_{1}\right), \mathrm{I}\left(L_{1}\right) \subset \mathrm{I}\left(L_{2}\right)$ and $\mathrm{N}\left(L_{1}\right) \subset \mathrm{N}\left(L_{2}\right)$. Repeating the last step if necessary produces a line 
segment $L=\left(p^{\prime}, q^{\prime}\right)$ with $\gamma \in \mathrm{N}(L), \mathrm{I}\left(L_{0}\right) \subset \mathrm{I}(L)$ and $\mathrm{N}\left(L_{0}\right) \subset \mathrm{N}(L)$. We can also assume that neither $p^{\prime}$ nor $q^{\prime}$ is contained in a facet of $\operatorname{MF}(\mathcal{Y} / S)$. So there are cones $\sigma_{p_{1}}, \sigma_{q_{1}} \in \operatorname{MF}_{\max }(\mathcal{Y} / S)$ with $p_{1} \in \operatorname{Int}\left(\sigma_{p_{1}}\right)$ and $q_{2} \in \operatorname{Int}\left(\sigma_{q_{1}}\right)$, so $L$ is indeed a test segment.

Lemma 8.9. Consider a connected component $\mathcal{C}$ of $\mathcal{M}$ and its closure $\overline{\mathcal{C}}$. Let $p_{0}, q_{0}$ be points in the interior $\overline{\mathcal{C}}^{o}$ of the closure and set $L_{0}=L\left(p_{0}, q_{0}\right)$. Let $\gamma \in \mathrm{MF}_{\max }(\mathcal{Y} / S)$. If $L_{0} \cap \gamma \neq \varnothing$, then there exist $\mathbb{Z}$-divisors $p$ and $q$ such that $L(p, q)$ is a nice test segment with $L \cap \gamma \neq \varnothing$. Also, $\mathrm{N}\left(L_{0}\right) \subset \mathrm{N}(L)$ and $\mathrm{I}\left(L_{0}\right) \subset \mathrm{I}(L)$.

Proof. We first show the existence of a test sequence $L=L(p, q)$ as claimed without the requirement that $p, q$ are $\mathbb{Z}$-divisors. Given data as in the statement of the lemma, by Lemma 8.8, there is a test segment $L_{1}$ with $\gamma \in \mathrm{N}\left(L_{1}\right)$. Arguing inductively, let $L_{i}$ be a test segment with $\gamma \in \mathrm{N}\left(L_{i}\right)$. Let $\sigma$ be a cone in $\mathrm{M}\left(L_{i}\right) \backslash \mathrm{N}\left(L_{i}\right)$. Again by Lemma 8.8, we find a test segment $L_{i+1}$ with $\mathrm{I}\left(L_{i}\right) \subset \mathrm{I}\left(L_{i+1}\right)$ and $\mathrm{N}\left(L_{i}\right) \subset \mathrm{N}\left(L_{i+1}\right)$ and $\left|\mathrm{N}\left(L_{i}\right)\right|<\left|\mathrm{N}\left(L_{i+1}\right)\right|$. Hence we obtain a sequence $\left\{L_{i}\right\}_{i}$ of test segments with $\left|\mathrm{N}\left(L_{i}\right)\right|<\left|\mathrm{N}\left(L_{i+1}\right)\right|$. As $\left|\mathrm{MF}_{\max }(\mathcal{Y} / S)\right|<\infty$, this is a finite sequence $\left\{L_{1}, L_{2}, \ldots, L_{n}\right\}$, and thus $\mathrm{M}\left(L_{n}\right)=\mathrm{N}\left(L_{n}\right)$. Setting $L=L_{n}$ proves the claim.

Now, given $L=L(p, q)$, perturbing a little, we can assume that $p, q$ are classes of $\mathbb{Q}$-divisors. Then there is an $n$ such that $L(n p, n q)$ is a test segment with the desired properties.

Theorem 8.10. Let $\mathcal{Y} \rightarrow S$ be a model of the DNV family of degree 2 . The closures $\overline{\mathcal{C}}_{i}, i=1, \ldots, 4$ of the connected components of $\mathcal{M}$ are convex cones. The collection

$$
\Sigma_{\mathcal{M}}=\left\{\sigma \subset \mathcal{M} \mid \sigma \text { is a face of some } \overline{\mathcal{C}}_{i}\right\}
$$

defines a finite fan of rational polyhedral cones.

Definition 8.11. The fan $\Sigma_{\mathcal{M}}$ is the secondary fan of $\mathcal{Y}$, denoted by $\operatorname{MF}^{2 n d}(\mathcal{Y} / S)$.

Proof of Theorem 8.10. All that remains to be shown is that the connected components of $\mathcal{M}$ have convex closures and that $\bar{C}_{i} \cap \bar{C}_{j} \in \Sigma_{\mathcal{M}}$. Let $\mathcal{C}$ be a connected component. It is enough to show that its interior $\mathcal{C}^{o}$ is convex. Let $p_{0}, q_{0}$ be points in $\mathcal{C}^{o}$. Let $\gamma$ be a maximal cone meeting $L=L\left(p_{0}, q_{0}\right)$. By Lemma 8.9, we find a nice $\overline{\mathcal{C}}$-test segment $L(p, q)$ with $\gamma \in \mathrm{N}(L(p, q))$, where $p$ and $q$ can be chosen to be classes of divisors. By Proposition 8.6, $\gamma \subset \overline{\mathcal{C}}$. Let $\partial \operatorname{Mov}(\mathcal{Y} / S)$ be the boundary of the moving cone. Now, we can decompose $\overline{\mathcal{C}}$ as

$$
\overline{\mathcal{C}}=\mathcal{C}^{0} \cup B_{1} \cup B_{2}
$$

where $B_{1} \subset \partial \operatorname{Mov}(\mathcal{Y} / S)$ and $B_{2} \subset \cup_{\tau \in \mathcal{F}}|\tau|$. where $\mathcal{F}$ is the set of all facets of type II of the Mori fan. By convexity of $\operatorname{Mov}(\mathcal{Y} / S)$ it follows that $L \subset \operatorname{Mov}(\mathcal{Y} / S)^{o}$. As we have just seen, all maximal cones $\gamma$ meeting $L=L\left(p_{0}, q_{0}\right)$ are contained in $\overline{\mathcal{C}}$. It follows that all facets $\tau$ met by $L$ are of type $\mathrm{I}$, as else the maximal cones containing $\tau$ would correspond to models of the DNV family that do not have the same class. So $L \cap B_{2}=\varnothing$ and thus $L \subset \mathcal{C}^{o}$, implying that $\mathcal{C}^{o}$ is convex. It follows from Lemma 8.7 that $\overline{\mathcal{C}}$ is convex. By Proposition 8.4, we obtain 4 maximal cones $\overline{\mathcal{C}}_{i}, i=1, \ldots, 4$. It remains to check that $\Sigma_{\mathcal{M}}$ is indeed a fan. This will follow from the following description of the $\overline{\mathcal{C}}_{i}$. Assume $\mathcal{C}_{1}=\mathcal{C}_{\mathscr{P}}$. From the construction, we immediately have the following description of the maximal cones. For each maximal cone $\overline{\mathcal{C}}_{i}$ the facets contained in $\partial \operatorname{Mov}(\mathcal{Y} / S)$ are unions of maximal cones in

$$
\cup_{\left\{\sigma \in \mathrm{MF}_{\max }(\mathcal{Y} / S) \mid \sigma \subset \overline{\mathcal{C}}_{i}\right\}} \partial \operatorname{Mov}(\mathcal{Y} / S) \cap \sigma .
$$

It follows from the convexity of the cones $\overline{\mathcal{C}}_{i}$, that each $\overline{\mathcal{C}}_{i}, i=2,3,4$ has a unique facet $H_{\mathcal{C}_{i}}$ meeting the interior of $\operatorname{Mov}(\mathcal{Y} / S) . H_{\mathcal{C}_{i}}$ is the union of type II facets that are contained in the $\overline{\mathcal{C}}_{i}$, formally

$$
H_{\mathcal{C}_{i}}=\cup_{\tau \in \mathcal{F}: \tau \subset \overline{\mathcal{C}_{i}}}|\tau| .
$$

For $i=1$, one has again the boundary facets and also the facets $H_{\mathcal{C}_{i}}$. Note that by definition of $\mathcal{M}$ the intersection of two cones of type $\mathscr{T}$ of $\Sigma_{\mathcal{M}}$ is at least of codimension 2 . It follows $\overline{\mathcal{C}}_{i} \cap \overline{\mathcal{C}}_{j} \in \Sigma_{\mathcal{M}}$. So $\Sigma_{\mathcal{M}}$ is 


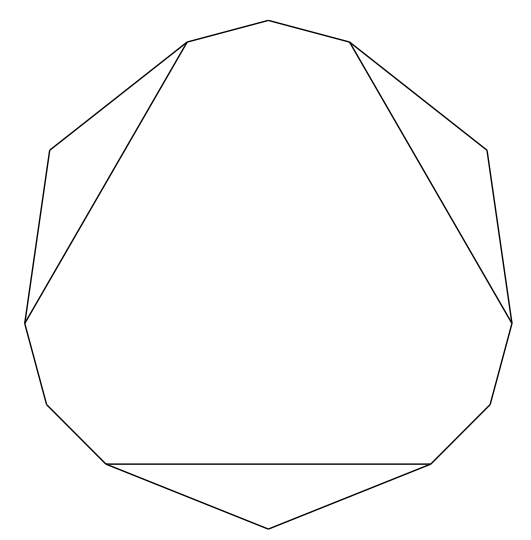

Figure 23. A schematic picture of $\operatorname{MF}^{2 n d}(\mathcal{Y} / S)$. The central cone is $\mathcal{C} \mathscr{P}$.

indeed a fan. Being a coarsening of a finite fan consisting of rational polyhedral cones, all cones of $\Sigma_{\mathcal{M}}$ are also rational polyhedral.

Remark 8.12. The $S_{3}$-action on $\mathcal{Y}_{\mathscr{P}}$ induces an action on the maximal cones of $\operatorname{MF}^{2 \mathrm{nd}}(\mathcal{Y} / S)$. Write $\overline{\mathcal{C}}_{i}, i=1, \ldots, 4$ for the maximal cones. Assume $\mathcal{C}_{1}=\mathcal{C}_{\mathscr{P}}$. As $\mathcal{Y}_{\mathscr{P}}$ has as unique associated cone, this action leaves $\mathcal{C}_{\mathscr{P}}$ invariant. Combining the arguments in Proposition 8.6 and Proposition 6.43 shows that the $S_{3}$-action is indeed a permutation action on the cones $\bar{C}_{i}, i=2, \ldots, 4$. Figure 23 provides a symbolic description of the structure of $\operatorname{MF}^{2 n d}(\mathcal{Y} / S)$.

\section{References}

[Ale02] V. Alexeev, Complete moduli in the presence of semiabelian group action, Ann. Math. (2) 155 (2002), no. 3, 611-708.

[AE21] V. Alexeev and P. Engel, Compact moduli of K3 surfaces, preprint arXiv:2101.12186 (2021).

[AET19] V. Alexeev, P. Engel, and A. Thompson, Stable pair compactification of moduli of K3 surfaces of degree 2, preprint arXiv:1903.09742v2 (2019).

[AL11] M. Artebani and A. Laface, Cox rings of surfaces and the anticanonical Iitaka dimension, Adv. Math. 226 (2011), no. 6, 5252-5267.

[Bad01] L. Badescu, Algebraic surfaces, Universitext, Springer, New York, NY, 2001.

[BHPV04] W. P. Barth, K. Hulek, C. A. M. Peters, and A. Van de Ven, Compact complex surfaces, Ergeb. Math. Grenzgeb., 3. Folge, vol. 4, Springer, Berlin, 2004.

[Car80] J. A. Carlson, Extensions of mixed Hodge structures. In: Journées de Géometrie Algébrique d'Angers, Juillet 1979, pp. 107-127, Sijthoff \& Noordhoff, Alphen aan den Rijn-Germantown, Md., 1980.

[Cor95] A. Corti, Recent results in higher-dimensional birational geometry. In: Current topics in complex algebraic geometry, pp. 35-56, Cambridge University Press, Cambridge, 1995.

[Deb01] O. Debarre, Higher-dimensional algebraic geometry, Universitext, Springer, New York, NY, 2001.

[Dol96] I. Dolgachev, Mirror symmetry for lattice polarized K3 surfaces, J. Math. Sci. 81 (1996), no. 3, 2599-2630.

[ES17] P. Engel and P. Smillie, The number of convex tilings of the sphere by triangles, squares, or hexagons, Geom. Topol. 22 (2018), no. 5, 2839-2864.

[Fri83] R. Friedman, Global smoothings of varieties with normal crossings, Ann. Math. (2) 118 (1983), 75-114. 
[FM83] R. Friedman and D. Morrison, The birational geometry of degenerations: an overview. In: The birational geometry of degenerations, pp. 1-29, Birkhäuser, Boston, MA, 1983.

[FS86] R. Friedman and F. Scattone, Type III degenerations of K3 surfaces, Invent. Math. 83 (1986), 1-39.

[GHK15] M. Gross, P. Hacking, and S. Keel, Moduli of surfaces with an anti-canonical cycle, Compos. Math. 151 (2015), no. 2, 265-291.

[GHKS] M. Gross, P. Hacking, S. Keel, and B. Siebert, Theta functions on K3 surfaces, in preparation.

[GHS15] V. Gritsenko, K. Hulek, and G. K. Sankaran, Moduli of K3 surfaces and irreducible symplectic manifolds. In: Handbook of moduli. Volume I, pp. 459-526, International Press, Somerville, MA; Higher Education Press, Beijing, 2015.

[GHS16] M. Gross, P. Hacking, and B. Siebert, Theta functions on varieties with effective anti-canonical class, preprint arXiv:1601.07081 (2016).

[GKZ94] I. M. Gelfand, M. M. Kapranov, and A. V. Zelevinsky, Discriminants, resultants, and multidimensional determinants, Birkhäuser Boston, Inc., Boston, MA, 1994.

[GW10] U. Görtz and T. Wedhorn, Algebraic geometry I. Schemes with examples and exercises. Vieweg + Teubner, Wiesbaden, 2010.

[Har77] R. Hartshorne, Algebraic geometry, Graduate Texts in Mathematics, vol. 52, Springer, New York, NY, 1977.

[HK00] Y. Hu and S. Keel, Mori dream spaces and GIT, Mich. Math. J. 48 (2000), 331-348.

[HKY20] P. Hacking, S. Keel, and T. Yue Yu, Secondary fan, theta functions and moduli of Calabi-Yau pairs, preprint arXiv:2008.02299 (2020).

[HLL20] K. Hulek, C. Lehn, and C. Liese, On the GHKS compactification of the moduli space of K3 surfaces of degree two, preprint arXiv:2010.06922 (2020).

[Huy16] D. Huybrechts, Lectures on K3 surfaces, Camb. Stud. Adv. Math., vol. 158, Cambridge University Press, Cambridge, 2016.

[Ill05] L. Illusie, Grothendieck's existence theorem in formal geometry. In: Fundamental algebraic geometry: Grothendieck's FGA explained, pp. 181-233, Math. Surv. Monogr., vol. 123, American Mathematical Society (AMS), Providence, RI, 2005.

[Kaw97] Y. Kawamata, On the cone of divisors of Calabi-Yau fiber spaces, Int. J. Math. 8 (1997), no. 5, 665-687.

[Kaw11] , Remarks on the cone of divisors. In: Classification of algebraic varieties, pp. 317-325, EMS Ser. Congr. Rep., Eur. Math. Soc., Zürich, 2011.

[KK98] V. S. Kulikov and P. F. Kurchanov, Complex algebraic varieties: Periods of integrals and Hodge structures. In: Algebraic geometry. III. Complex algebraic varieties, algebraic curves and their Jacobians, pp. 1-217, Encyclopaedia Math. Sci., vol. 36, Springer, Berlin, 1998.

[Kle66] S. L. Kleiman, Toward a numerical theory of ampleness, Ann. Math. (2) 84 (1966), 293-344.

[KM98] J. Kollár and S. Mori, Birational geometry of algebraic varieties. With the collaboration of C. H. Clemens and A. Corti. Cambridge Tracts in Mathematics, vol. 134, Cambridge University Press, Cambridge, 1998.

[Kol95] J. Kollár, Rational curves on algebraic varieties, Ergeb. Math. Grenzgeb., 3. Folge, vol. 32, SpringerVerlag, Berlin, 1995. 
[Kul77] V. S. Kulikov, Degenerations of K3 surfaces and Enriques surfaces, Math. USSR, Izv. 11 (1977), 957-989.

[Laz04] R. Lazarsfeld, Positivity in algebraic geometry. I. Classical setting: line bundles and linear series, Ergeb. Math. Grenzgeb., 3. Folge, vol. 48, Springer, Berlin, 2004.

[Laz08] R. Laza, Triangulations of the sphere and degenerations of K3 surfaces, preprint arXiv:0809.0937 (2008).

[Laz16] _ The KSBA compactification for the moduli space of degree two K3 pairs, J. Eur. Math. Soc. (JEMS) 18 (2016), no. 2, 225-279.

[Lie17] C. Liese, The KSBA compactification of degree 2 K3 pairs: a toroidal interpretation, Ph.D. thesis, Universität Hamburg, 2017. Available from https://ediss.sub.uni-hamburg.de/bitstream/ ediss/7787/1/Dissertation.pdf.

[LO16] R. Laza and K. O'Grady, Birational geometry of the moduli space of quartic K3 surfaces, Compos. Math. 155 (2019), no. 9, 1655-1710.

[LO18] _ GIT versus Baily-Borel compactification for quartic K3 surfaces. In: Geometry of moduli, pp. 217-283, Abel Symp., vol. 14, Springer, Cham, 2018.

[LO21] _ GIT versus Baily-Borel compactification for K3's which are double covers of $\mathbb{P}^{1} \times \mathbb{P}^{1}, A d v$. Math. 383 (2021), article No. 107680.

[Loo81] E. Looijenga, Rational surfaces with an anti-canonical cycle, Ann. Math. (2) 114 (1981), 267-322.

[Loo86]__ New compactifications of locally symmetric varieties. In: Proceedings of the 1984 Vancouver conference in algebraic geometry, pp. 341-364, CMS Conf. Proc., vol. 6, Amer. Math. Soc., Providence, RI, 1986.

[LY96a] B. H. Lian and S.-T. Yau, Arithmetic properties of mirror map and quantum coupling, Commun. Math. Phys. 176 (1996), no. 1, 163-191.

[LY96b] _ Mirror maps, modular relations and hypergeometric series. II, Nuclear Phys. B Proc. Suppl. 46 (1996), 248-262.

[Mat89] H. Matsumura, Commutative ring theory, Camb. Stud. Adv. Math., vol. 8, Cambridge University Press, Cambridge, 1989.

[Mat02] K. Matsuki, Introduction to the Mori program, Universitext, Springer, New York, NY, 2002.

[MM83] R. Miranda and D. Morrison, The minus one theorem. In: The birational geometry of degenerations, pp. 173-259, Birkhäuser, Boston, MA, 1983.

[Nik79] V. V. Nikulin, Integer symmetric bilinear forms and some of their geometric applications, Izv. Akad. Nauk SSSR Ser. Mat. 43 (1979), no. 1, 111-177, 238.

[Nik81] _ Quotient-groups of groups of automorphisms of hyperbolic forms by subgroups generated by 2-reflections. Algebro-geometric applications. In: Current problems in mathematics, vol. 18, pp. 3-114, Akad. Nauk SSSR, Vsesoyuz. Inst. Nauchn. i Tekhn. Informatsii, Moscow, 1981.

[Nik14] _ _ Elliptic fibrations on K3 surfaces, Proc. Edinb. Math. Soc. (2) 57 (2014), no. 1, 253-267.

[Ols12] M. Olsson, Compactifications of moduli of abelian varieties: an introduction. In: Current developments in algebraic geometry, pp. 295-348, Math. Sci. Res. Inst. Publ., vol. 59, Cambridge Univ. Press, Cambridge, 2012.

[Pin77] H. Pinkham, Singularités exceptionnelles, la dualité étrange d'Arnold et les surfaces K3, C. R. Acad. Sci. Paris Sér. A, 284 (1977), no. 11, 615-618. 
[PP81] U. Persson and H. Pinkham, Degeneration of surfaces with trivial canonical bundle, Ann. Math. (2) 113 (1981), 45-66.

[Sca87] F. Scattone, On the compactification of moduli spaces for algebraic K3 surfaces, Mem. Amer. Math. Soc., vol. 374, American Mathematical Society, Providence, RI, 1987.

[Sha80] J. Shah, A complete moduli space for K3 surfaces of degree 2, Ann. Math. (2) 112 (1980), 485-510.

[Sho96] V. V. Shokurov, 3-Fold log models, J. Math. Sci. 81 (1996), no. 3, 2667-2699.

[TVV11] D. Testa, A. Várilly-Alvarado, and M. Velasco, Big rational surfaces, Math. Ann. 351 (2011), no. 1, 95-107.

[Tho14] A. Thompson, Degenerations of K3 surfaces of degree two, Trans. Amer. Math. Soc. 366 (2014), no. 1, 219-243.

[Voi93] C. Voisin, Miroirs et involutions sur les surface K3, Astérisque 218 (1993), 273-323.

[Wah76] J. M. Wahl, Equisingular deformations of normal surface singularities. I, Ann. Math. (2) 104 (1976), 325-356.

[Zhu18] Y. Zhu, Compactification of the moduli of polarized abelian varieties and mirror symmetry, Trans. Amer. Math. Soc. 370 (2018), no. 3, 1693-1758. 